\title{
Improvement of data quality for Diffusion Kurtosis Imaging and application to clinical neurological research
}

Citation for published version (APA):

André, É. (2014). Improvement of data quality for Diffusion Kurtosis Imaging and application to clinical neurological research. [Doctoral Thesis, Maastricht University]. Maastricht University. https://doi.org/10.26481/dis.20140619ea

Document status and date:

Published: 01/01/2014

DOI:

10.26481/dis.20140619ea

Document Version:

Publisher's PDF, also known as Version of record

Please check the document version of this publication:

- A submitted manuscript is the version of the article upon submission and before peer-review. There can be important differences between the submitted version and the official published version of record.

People interested in the research are advised to contact the author for the final version of the publication, or visit the DOI to the publisher's website.

- The final author version and the galley proof are versions of the publication after peer review.

- The final published version features the final layout of the paper including the volume, issue and page numbers.

Link to publication

\footnotetext{
General rights rights.

- You may freely distribute the URL identifying the publication in the public portal. please follow below link for the End User Agreement:

www.umlib.nl/taverne-license

Take down policy

If you believe that this document breaches copyright please contact us at:

repository@maastrichtuniversity.nl

providing details and we will investigate your claim.
}

Copyright and moral rights for the publications made accessible in the public portal are retained by the authors and/or other copyright owners and it is a condition of accessing publications that users recognise and abide by the legal requirements associated with these

- Users may download and print one copy of any publication from the public portal for the purpose of private study or research.

- You may not further distribute the material or use it for any profit-making activity or commercial gain

If the publication is distributed under the terms of Article $25 \mathrm{fa}$ of the Dutch Copyright Act, indicated by the "Taverne" license above, 


\title{
Improvement of data quality for Diffusion Kurtosis Imaging and application to clinical neurological research
}

\author{
Elodie André
}


(c) Elodie André, 2014.

The work presented in this thesis was funded by the EU within the PEOPLE Programme (FP7): Initial Training Networks (FP7-PEOPLE-ITN-2008), Grant Agreement No. 238593 NEUROPHYSICS, in which Maastricht University, Université de Liège, Forschungszentrum Juelich GmbH and GlaxoSmithKline Ltd are the network partners. The $\mathrm{PhD}$ was mainly conducted at Université de Liège. 


\section{Improvement of data quality for Diffusion Kurtosis Imaging and application to clinical neurological research}

\section{DISSERTATION}

to obtain the joint degree of Doctor at

University of Liège and Maastricht University,

on the authority of the Rector Magnifici, Prof. B. Rentier and Prof.

Dr. L.L.G Soete

in accordance with the decision of the Board of Deans,

to be defended in public

on Thursday $19^{\text {th }}$ of June 2014 at 14:00 hours

in Maastricht

by

Elodie André 


\section{Supervisors}

Prof. Dr. N.J. Shah, Maastricht University

Ir. Dr. C. Phillips, University of Liège

\section{Co-supervisors}

Dr. E. Balteau, University of Liège

Dr. F. Grinberg, Forschungszentrum Juelich GmbH

\section{Assessment Committee}

Prof. Dr. J. Verly, University of Liège, Chairman

Prof. Dr. E. Formisano, Maastricht University

Dr. A. Leemans, Utrecht University

Dr. S. Mohammadi, University College London 


\section{Contents}

\begin{tabular}{ll}
\hline Abstract & 7
\end{tabular}

$\begin{array}{ll}\text { Résumé } & 9\end{array}$

$\begin{array}{lll}1 & \text { Introduction } & 11\end{array}$

2 Diffusion MRI: From water diffusion to brain microstructure 13

2.1 Principles of diffusion $\ldots \ldots \ldots \ldots \ldots$

2.2 Diffusion in biological tissue $\ldots \ldots \ldots \ldots$

2.2 .1 Central nervous system . . . . . . . . . . . . . 14

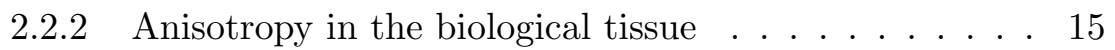

2.3 Acquisition of diffusion images $\ldots \ldots \ldots \ldots \ldots$

$2.3 .1 \quad$ Diffusion weighting in MRI . . . . . . . . . . . 15

2.3 .2 Distortions and artefacts caused by the presence of eddy currents . . . . . . . . . . . . . . . . 19

2.3 .3 Other artefacts . . . . . . . . . . . . . . . . 20

2.4 Data modelling . . . . . . . . . . . . . . . . . . . . . . . . 22

2.4 .1 Diffusion weighted imaging . . . . . . . . . . . 22

2.4 .2 Diffusion tensor imaging . . . . . . . . . . . . . . . 22

2.4 .3 Diffusion kurtosis imaging . . . . . . . . . . . . . . 26

$2.4 .4 \quad$ Other Non-Gaussian approches . . . . . . . . . . . . 29

\begin{tabular}{|ll|}
3 & Motion correction for diffusion experiments \\
\hline
\end{tabular}

3.1 Introduction . . . . . . . . . . . . . . . 31

$3.1 .1 \quad$ Coregistration in MRI . . . . . . . . . . . . . . . 32

3.1 .2 Coregistration of diffusion weighted images . . . . . . 35

3.1.3 $\quad$ Objectives . . . . . . . . . . . . . . . . . 37

3.2 Methods . . . . . . . . . . . . . . . . . 37

$3.2 .1 \quad$ Iterative model-based method . . . . . . . . . . . 37

3.2 .2 Method assessment . . . . . . . . . . . . . . . . . 40

3.2 .3 Method comparison . . . . . . . . . . . . . . . . . . 41 
3.3 Results. . . . . . . . . . . . . . . . . . . . . . . . . . . . . . 42

3.3.1 Synthetic data: non-DW images interleaved with the DW images . . . . . . . . . . . . . . . . . 44

3.3 .2 Synthetic data: non-DW images at the beginning of

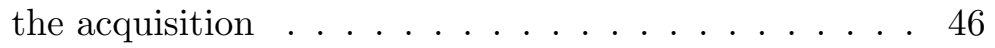

3.3 .3 Stopping criterion $\ldots \ldots \ldots \ldots$

3.3 .4 Real data . . . . . . . . . . . . . . . . . . . . . . . 49

3.4 Discussion . . . . . . . . . . . . . . . . . . . . . 50

$\begin{array}{|ll|}4 & \text { Noise correction for DKI experiments }\end{array}$

4.1 Introduction . . . . . . . . . . . . . . . . . . . . 53

4.2 Signal distribution in magnitude MR images . . . . . . . . . 55

4.2 .1 Single channel coil: Rician distribution . . . . . . . . 55

$4.2 .2 \quad$ Multichannels coil: Noncentral $\chi$ distribution . . . . . 55

4.3 Noise correction methods . . . . . . . . . . . . . . . . 57

$4.3 .1 \quad$ Noise estimation . . . . . . . . . . . . . . . . . 57

4.3 .2 Image denoising: non local mean filtering . . . . . . . 59

$4.3 .3 \quad$ First method for noise bias correction: the first moment correction . . . . . . . . . . . . . . . . . 60

$4.3 .4 \quad$ Second method for noise bias correction: Power image correction . . . . . . . . . . . . . . 60

4.4 Experimental protocol $\ldots \ldots \ldots \ldots 1$

$4.4 .1 \quad$ Intra-subject inter-session variability . . . . . . . . . . 61

4.4 .2 Inter-subject variability $\ldots \ldots \ldots$. . . . . . . . . 62

4.4 .3 Ethics statement . . . . . . . . . . . . . . . . . . . 62

4.5 Global data processing . . . . . . . . . . . . . . . . . 62

4.5 .1 Data processing . . . . . . . . . . . . . . . . . 62

$4.5 .2 \quad$ Assessment of intra-subject variability . . . . . . . . . 64

$4.5 .3 \quad$ Assessment of inter-subject variability . . . . . . . . . 64

4.6 Results . . . . . . . . . . . . . . . . . . . . . 66

4.6 .1 Effect of noise correction. . . . . . . . . . . 66

4.6 .2 Intra-subject variability $\ldots \ldots \ldots \ldots$

4.6 .3 Inter-subject variability $\ldots \ldots \ldots \ldots . \ldots . \ldots 71$

4.7 Discussion . . . . . . . . . . . . . . . . . . . . . . 73

4.7 .1 Comparison of the different correction schemes . . . 73

$4.7 .2 \quad M K$ variability $\ldots \ldots \ldots \ldots \ldots \ldots$

$4.7 .3 \quad$ Applicability to clinical studies . . . . . . . . . . . 75

4.7 .4 Limitations . . . . . . . . . . . . . . . . . . 75

4.8 Conclusion $\ldots \ldots \ldots \ldots \ldots \ldots \ldots$ 
5 Medical application to Parkinson disease 83

5.1 Introduction . . . . . . . . . . . . . . . . . 83

5.2 Methods . . . . . . . . . . . . . . . . . . 84

5.2 .1 Population . . . . . . . . . . . . . . . 84

5.2 .2 Data processing . . . . . . . . . . . . . . . . 85

5.2 .3 Statistical analysis $\ldots \ldots \ldots \ldots$. . . . . . . . . . 87

5.3 Results . . . . . . . . . . . . . . . . . . . 88

$5.3 .1 \quad$ Cognitive evaluation . . . . . . . . . . . . 88

5.3 .2 Voxel based analysis of diffusion parameters . . . . . . 88

5.4 Discussion $\ldots \ldots \ldots \ldots \ldots \ldots$

5.5 Conclusion $\ldots \ldots \ldots \ldots \ldots \ldots$

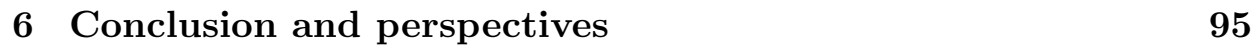

\begin{tabular}{ll}
\hline Abbreviations & 97
\end{tabular}

\begin{tabular}{ll}
\hline Bibliography & 105
\end{tabular}

\begin{tabular}{ll}
\hline Acknowledgments & 119
\end{tabular}

$\begin{array}{ll}\text { Curriculum vitae } & 121\end{array}$ 


\section{Abstract}

Understanding human brain function and dysfunction is one of the major challenges of our century. One of the most popular methods to achieve this goal is in vivo magnetic resonance imaging. In particular, diffusion-weighted (DW) imaging has become a standard tool to non-invasively study white matter structure in vivo.

The main contributions of this work can be divided in two parts. The first part deals with the development of pre-processing methods to improve image quality and the accuracy of diffusion tensor and diffusion kurtosis-derived parameters. First, we describe and evaluate a novel method to correct data misalignment due to subject motion. Using an iterative model-based approach, individual diffusion images are realigned to their own theoretical pair, rather than to the unweighted image. A recently developed advanced measure of tensor distance was used as a stopping criterion. The accuracy of the method is tested via a simulated diffusion tensor imaging data set. We have shown here that our procedure is a reliable and efficient way to correct subject motion during DW acquisitions, and that with a proper acquisition setup, it performs better than standard coregistration procedures.

Next, we studied the influence of noise in diffusion kurtosis imaging. Two noise correction approaches are proposed and applied to a pool of 25 subjects to evaluate inter-subject variability and the impact of noise correction. Additionally, data were acquired on a single subject with different head positions within the coil and different acquisition scheme to evaluate the impact of noise correction on within-subject variability. Results show a strong impact of noise correction on the estimated mean kurtosis, while the estimation of fractional anisotropy and mean diffusivity were less affected. Both within- and between-subject signal-to-noise (SNR) related variability of the mean kurtosis estimate is considerably reduced after correction for the noise bias, leading to more accurate and reproducible measures. In this work, we have proposed a straightforward method that improves the accuracy of diffusion kurtosis metrics. Diffusion kurtosis imaging acquisitions at higher spatial resolution are made possible, which increases the chances to make valuable inferences in group analysis. 
The second part of this thesis deals with a clinical application of these methods. A large group of patients with early-stage Parkinson's disease was investigated with diffusion kurtosis imaging and compared with a group of age- and sex-matched healthy volunteers using voxel-based analysis. Kurtosis metrics show more sensitivity to white matter changes than standard diffusion metrics. Significant differences were found in posterior cerebral areas as well as subcortical regions like the putamen, and are therefore promising results. 


\section{Résumé}

Comprendre le fonctionnement et le dysfonctionnement du cerveau humain est l'un des grands défis de ce siècle. Pour atteindre ce but, l'imagerie par résonance magnétique (IRM) in vivo est devenue une technique incontournable. En particulier, l'IRM de diffusion est aujourd'hui un outil standard et non invasif pour étudier la structure de la matière blanche in vivo.

Les principales constributions de ce travail de thèse se divisent en deux parties. Dans une première partie, deux nouvelles méthodes pour le prétraitement des images sont développées afin d'améliorer la qualité de cellesci. Ces méthodes permettront également d'augmenter la reproductibilité et la précision des paramètres dérivés des tenseurs de diffusion et de kurtosis. Tout d'abord, nous présentons et évaluons une nouvelle méthode pour recaler les images, initialement décalées à cause des mouvements du sujet. Via une approche itérative et qui repose sur un modèle, chaque image de diffusion est recalée individuellement sur sa propre paire théorique plutôt que sur l'image non pondérée en diffusion. Comme critère d'arrêt, nous avons utilisé une measure de distance entre deux tenseurs. Un set de données de tenseurs de diffusion a été simulé pour tester la performance de cette méthode. Nous avons démontré que notre procédure est une technique fiable et efficace pour corriger les effets dus aux mouvements du sujet pendant l'acquisition des images de diffusion. Nous avons également mis en évidence que cette méthode, utilisée avec des paramètres d'acquisition adéquats, permet d'obtenir de meilleurs résultats par rapport aux méthodes standard de recalage.

Ensuite, toujours pour améliorer la qualité des images, nous avons étudié l'influence du bruit dans le cadre de l'imagerie du tenseur de kurtosis. Deux méthodes de correction du bruit sont proposées et appliquées sur les données acquises sur 25 sujets afin d'évaluer la variabilité inter-sujets et l'impact de la correction du bruit sur cette variabilité. De plus, plusieurs sets de données ont été acquis sur un même sujet, en faisant varier d'une part la position de la tête à l'intérieur de l'antenne et d'autre part les paramètres d'acquisition, afin d'étudier l'impact de la correction du bruit sur la variabilité intra-sujet. Les résultats montrent un effet très important du bruit 
sur l'estimation du coefficient de kurtosis moyen. Par contre cet effet est relativement plus faible sur l'estimation de l'anisotropie fractionnelle et de la diffusivité moyenne. Après correction du bruit, la dépendance du coefficient moyen de kurtosis avec le rapport signal sur bruit, ainsi que de la variabilité intra- et inter-sujets, sont considérablement réduites, conduisant à des mesures plus justes et reproductibles. Nous avons donc proposé ici une méthode simple qui améliore la justesse et la précision des métriques dérivées des tenseurs de kurtosis, indépendemment du niveau de bruit. Il est donc possible d'augmenter la résolution spatiale et ainsi d'augmenter les chances de trouver des différences en comparant deux groupes de sujets. Dans une deuxième partie, nous avons appliqués les méthodes développées dans la première partie à une étude de recherche clinique. Un groupe de patients diagnostiqués à un stade pécoce de la maladie de Parkinson a suivi un protocole d'acquisition d'imagerie du tenseur de kurtosis et les données ont été comparées voxel par voxel avec celles acquises dans un groupe de sujets sains, avec une répartition semblable de l'âge et du sexe. Les paramètres dérivés du tenseur de kurtosis sont plus sensibles aux changements de la structure de la matière blanche que les paramètres standard dérivés du tenseur de diffusion. Des différences significatives ont été trouvées dans les régions cérébrales postérieures ainsi que dans les régions sous-corticales comme le putamen. Les résultats sont donc prometteurs. 


\section{Chapter 1}

\section{Introduction}

Understanding the human brain is one of the big challenges of our time. From the first known description of the brain by the Egyptians in the 17th century BC to now, huge steps have been overcome, and most of them only in the past few decades or even years. As an example, the basic concept of the neuron as a discrete element has been confirmed and generally accepted only in the fifties thanks to electron microscopy. The development of magnetic resonance imaging (MRI) has been a huge step: for the first time it has been possible to investigate both the brain's function and structure in vivo.

Diffusion weighted (DW) imaging in particular is one of the most recent advanced MRI technique, which has been successfully applied to human brain for the first time in the 80's (Le Bihan et al., 1986). Since then this technique has been developed to a large extent and is one of the most commonly used MRI techniques. By measuring the small displacements of water molecules in the brain, it is possible to detect its underlying microstructure and to "navigate" into the brain without any invasive procedure. Before the development of DW imaging, the only possible way to observe the complexity of white matter (WM) fibers was via post-mortem studies. As an example, some results obtained using diffusion tractography, used to track a fiber along its whole length, are shown in Figure 1.1. Diffusion MRI has thus opened a window toward new opportunities to characterize normal and diseased brains. In particular, diffusion offers the potential to investigate WM organisation in a number of diseases such as stroke, aging, dementia, schizophrenia, multiple sclerosis etc.

This is in this context that the objectives of this thesis are placed. The 
a)

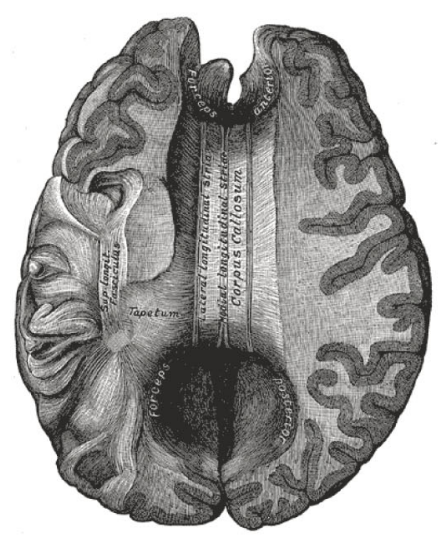

b)

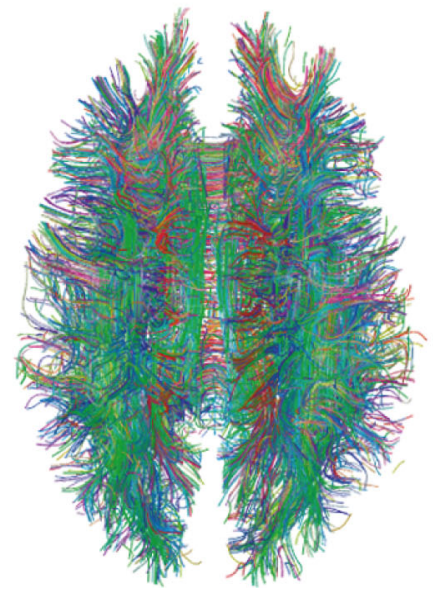

Figure 1.1: a) Brain representation of the corpus callosum (lithograph plate from the 20th U.S. edition of Gray's Anatomy of the Human Body). b) Similar view using diffusion imaging tractography. Image from Gigandet et al. (2008)

goal of this thesis was first to improve the quality of the diffusion data in order to improve reliability and accuracy of the result. Second, these improvements were applied to diffusion kurtosis data in the particular case of Parkinson's disease to investigate kurtosis as a potential biomarker. This work is organised as follows:

Chapter 2 is an introduction to diffusion MRI. After describing the general concept of the diffusion phenomenon and its application to biological tissues, we will go through diffusion MRI acquisition and data modeling. General physics MRI is assumed to be understood by the reader.

Chapter 3 is related to the development of an improved motion correction procedure for diffusion weighted imaging.

Chapter 4 is dedicated to the development of a simple and efficient noise correction procedure for data acquired with multi-channel coils and its impact of diffusion kurtosis.

In chapter 5, a group of patients with Parkinson's disease is investigated with diffusion kurtosis imaging and data are compared to a group of healthy volunteers. 


\section{Chapter 2}

\section{Diffusion MRI: From water diffusion to brain microstructure}

\subsection{Principles of diffusion}

Molecular diffusion is a mass transport process, which describes movement of particles, both at macroscopic and microscopic level. The main feature of this transport process as compared to convection or conduction is the absence of bulk motion. The phenomenon is well illustrated by a daily life situation: when pouring a drop of syrup into water both liquids will mix without stirring until the concentrations of both liquids are homogeneous in the glass. This macroscopic phenomenon was theoretically explained by Adolf Fick in 1855. The so-called "Fick's first law", which relates the flux of the given molecule to the concentration gradient of the same molecule, is given by:

$$
J(x, t)=-D \frac{\partial C(x, t)}{\partial x}
$$

where the $J$ is the flux of the molecule of interest, $C$ its concentration , $D$ the diffusion coefficient, $t$ represents the time and $x$ the position. The "Fick's second law" describes how molecular diffusion makes the concentration change with time:

$$
\frac{\partial C(x, t)}{\partial t}=-D \frac{\partial C(x, t)}{\partial^{2} x}
$$

The diffusion coefficient corresponds to the speed with which the molecules spread on a surface area by unit of time. It depends on the temperature and the viscosity of both the medium and the diffusing molecules. For example, the diffusion coefficient of water in water, also called self-diffusion, at $25^{\circ} \mathrm{C}$ is $2.3 \times 10^{-3} \mathrm{~mm}^{2} / \mathrm{s}$. 
Diffusion can also be described at the microscopic level by considering the "random walks". For the naked eye, nothing seems to happen in a glass of water. However, under a microscope, water molecules are moving around due to thermic energy. This is also called Brownian motion, as a reference to the botanist Brown, who was the first one to observe such diffusion process when observing particles extracted from pollen grains under the microscope (Brown, 1828). Some years later and independently, Albert Einstein described theoretically this displacement (Einstein, 1905). According to his work, the probability of a molecule to go from the position $r_{0}$ to the position $r$ during the time $t$ is given by:

$$
P\left(r \mid r_{0}, t\right)=(4 \pi D t)^{-3 / 2} \exp \left(-\frac{\left(r-r_{0}\right)^{2}}{4 D t}\right)
$$

The mean displacement is null $(<r>=0)$ but the squared root of the displacement is proportional to both the diffusion coefficient and the time of diffusion as described by the Einstein equation in one dimension:

$$
<r^{2}>=2 D t
$$

\subsection{Diffusion in biological tissue}

\subsubsection{Central nervous system}

The human nervous system is organised in two main components: the central nervous system and the peripheral nervous system. The former includes the brain and the spinal cord and receives sensory input and controls behaviour. The latter includes everything that conveys information from the brain to the organs and vice-versa. All the exchanges of information, between brain and organs and within the brain are done via the neurons. The brain itself contains over 100 billion neurons and each neuron may be connected up to 10 thousand neurons, which makes the study of the brain highly complicated. Usually, brain tissues are separated in three categories: the white matter (WM), the grey matter (GM) and the cerebrospinal fluid (CSF). The GM is formed by the bodies of the neurons, where the information is generated. The WM is formed by the axons, which are grouped in bundles and convey the information from one neuron to another. The CSF is a normally clear and cell-free fluid that fills the ventricular system of the central nervous system. In summary, the organisation of WM fibres defines the structural connectivity of the brain, which in turn constraints its functional dynamic. Therefore, being able to study WM in vivo is a very important challenge and has been made possible thanks to diffusion MRI. 


\subsubsection{Anisotropy in the biological tissue}

From the early diffusion studies, diffusion in brain living tissue has been shown to be anisotropic in animals (Moseley et al. 1990) and humans (Chenevert et al., 1990). The diffusion of water molecules in brain tissues is constrained by their structures, which participate to the observed anisotropy at different scales. The main cells surrounding neurons are illustrated in Figure 2.1(a). Neurons represent around $10 \%$ of the total amount of brain cells but the brain is also formed by other type of cells and structures such as oligodendrocytes, which are making the myelin sheaths, astrocytes and microglial cells, which are supporting cells for neurons, microtubules or neurofilaments, etc. Furthermore, most of the axons in the the brain are wrapped by layers of myelin, as shown in Figure 2.1(b), in order to increase information transfer speed. The axonal structure and the myelination (Beaulieu, 2002) are the main reasons for the anisotropy observed in the brain. Electron micrographs of longitudinal and transversal sections of an axon bundle are shown in Figure 2.1(c): the axonal membrane acts like a barrier and limits the diffusion of water molecule in the axial direction, while the diffusion in the direction of the axon is higher. In brain tissues, one can distinguish three different types of diffusion: free, hindered and restricted. Both free and hindered diffusion follow the Gaussian displacement distribution (Johansen-Berg and Behrens, 2009). Free diffusion occurs when absolutely no barriers are present. In brain tissue, this intrinsic diffusivity is extremely difficult to measure with imaging techniques, as it requires very short diffusion time (see section 2.3). Hindered diffusion occurs when diffusion is free in a given time-range and then is reduced by various barriers. That is what we can observe in the brain tissue, with the standard diffusion times. The measured diffusion coefficient is then called apparent diffusion coefficient (ADC), as it is not exactly the free diffusion coefficient. Finally restricted diffusion occurs in closed area, inside a cell for example, where the course of the molecules is limited by the diameter of this cell. Different models have been proposed to describe these aspects. They are described in section 2.4.

\subsection{Acquisition of diffusion images}

\subsubsection{Diffusion weighting in MRI}

In 1950, Carr and Purcell showed that the nuclear magnetic resonance spin echoes discovered by Hahn (1950) could be used as a direct method to measure diffusion coefficient in a sample. Later on, in 1965, Stejskal and Tanner (1965) developed the Pulsed Gradient Spin Echo (PGSE) sequence, which 

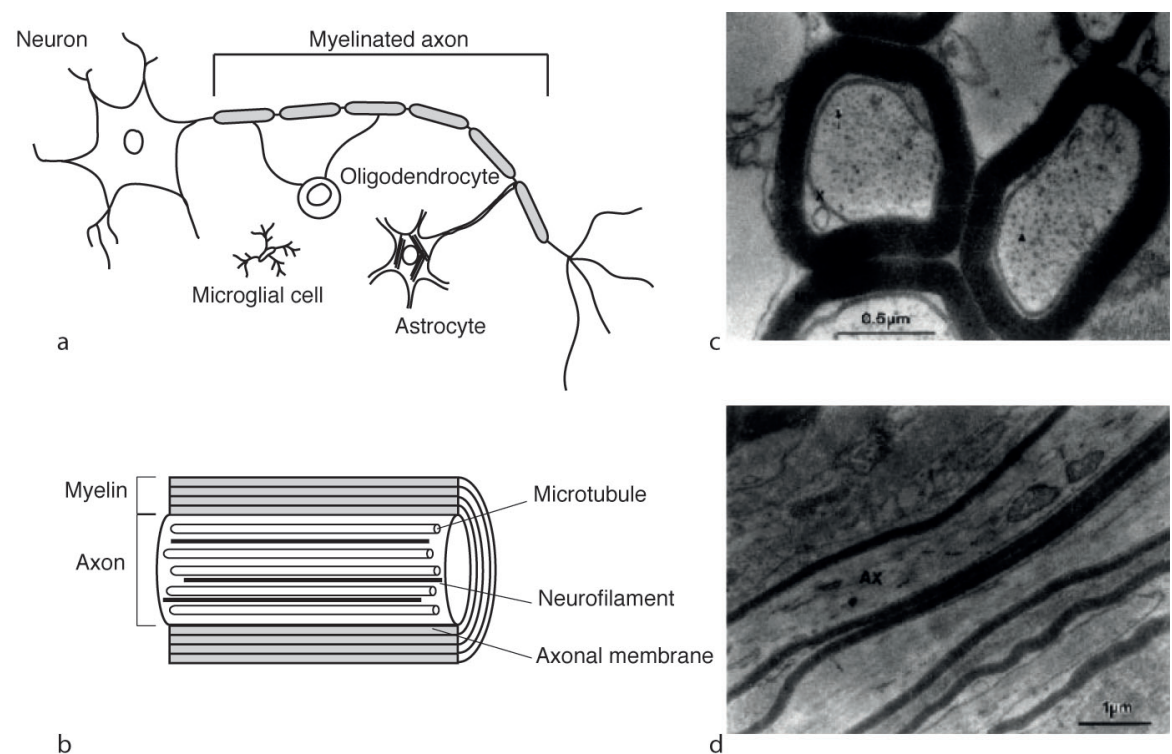

b

Figure 2.1: The main components of the central nervous system. a) Schematic representation of a neuron and the main cellular elements of the white matter, adapted from Johansen-Berg and Behrens (2009), b) Shematic representation of an axon surrounded by myelin. Electron myograph of c) axonal section and d) longitudinal section of an optic nerve. b), c) and d) are reproduced from Beaulieu (2002) with permissions (Copyright (C) 2002 John Wiley 85 Sons, Ltd.)

is still the most common DW imaging sequence. The sequence diagram is shown in Figure 2.2 and the following detailed explanations of DW imaging are based on that sequence.

\section{Diffusion encoding}

The diffusion PGSE sequence is based on a pair of dephasing/rephasing gradients pulses: after the $90^{\circ}$ radiofrequency (RF) pulse, spins of water molecules start to dephase during the first diffusion gradient, as described in Figure 2.2. In the case of narrow pulse approximation, one assumes that $\delta<<\Delta$, where $\delta$ is the diffusion gradient duration and $\Delta$ the time between the beginning of the first and the second gradient directions. In other words, one assumes that the molecular displacement is negligible during the gradient application. The spins are then labeled in one specific direction by their phase $\phi_{1}$ which is defined as:

$$
\phi_{1}=\gamma G \int_{0}^{\delta} r d t=\gamma G r_{1} \delta
$$




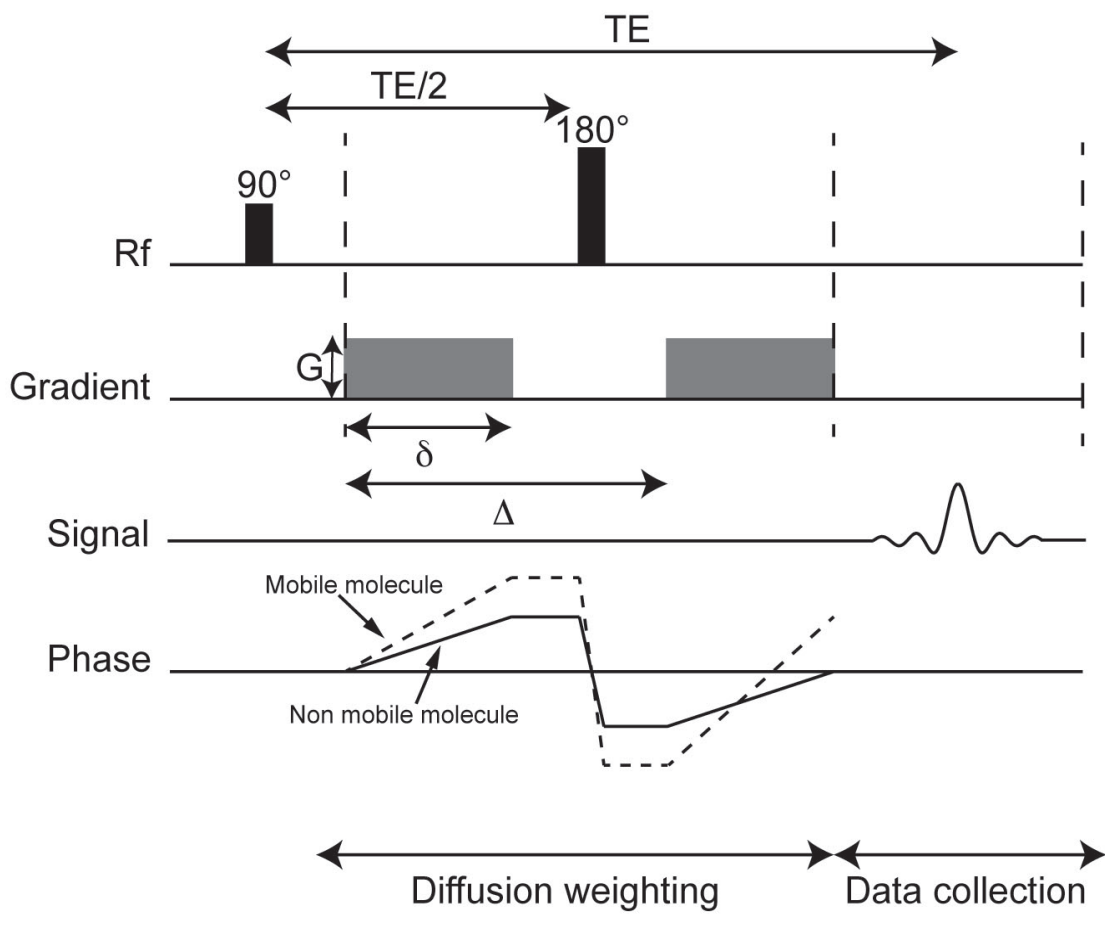

Figure 2.2: The conventional PGSE sequence. The first row represents the radiofrequency $(R f)$ pulses, the second the applied gradients (here for one single direction), the third one the signal and the last one represents the evolution of the phase for two molecules: a non-mobile one (solid line) and a mobile one (dashed line). TE is the echo time, $G$ the gradient strength, $\delta$ the gradient duration and $\Delta$ the diffusion time.

where $\gamma$ is the gyromagnetic ratio and $\delta$ the gradient pulse duration, as described in Figure 2.2. Then the phase is inversed with a $180^{\circ}$ pulse and another gradient with the same strength is applied. The phase $\phi_{2}$ acquired during the second gradient is:

$$
\phi_{2}=\gamma G \int_{\Delta}^{\Delta+\delta} r d t=\gamma G r_{2} \delta
$$

The total dephasing is then equal to:

$$
\phi_{1}-\phi_{2}=\gamma G\left(r_{1}-r_{2}\right) \delta
$$

If molecules are stationary, $r_{1}-r_{2}=0$ and spins rephase completely, meaning that no signal attenuation is observed. This is illustrated by the solid phase line in Figure 2.2. However, if molecules moved, $\left|r_{1}-r_{2}\right|>0$, spins 
rephase partially, as shown with the dashed line in Figure 2.2. It results in a signal attenuation.

In the case of free diffusion, the diffusion propagator is a Gaussian function.

In that case, the MR signal attenuation $S / S_{0}$ is given by (Stejskal and Tanner, 1965):

$$
\log \left(\frac{S}{S_{0}}\right)=-(\gamma \delta G)^{2} D\left(\Delta-\frac{\delta}{3}\right)=-b D
$$

where $\mathrm{b}$ is the so-called $b$-value and is proportional to the gradient strength and the diffusion time. It is used as a marker of diffusion weight and can be analytically expressed for rectangular gradients by:

$$
b=(\gamma \delta G)^{2}\left(\Delta-\frac{\delta}{3}\right)
$$

For a typical diffusion acquisition, this sequence is applied in different directions, by using appropriate combinations of diffusion gradients.

\section{Data collection}

Data collection is usually done using an echo planar imaging (EPI) readout, as described in Figure 2.3. The choice of EPI is mainly motivated by the speed of the acquisition.

a)

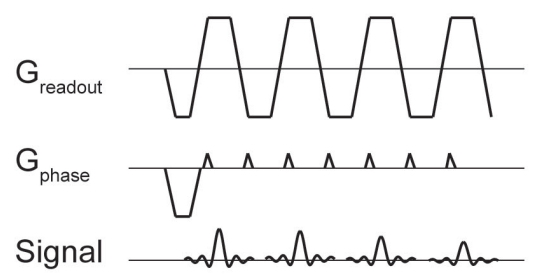

b)

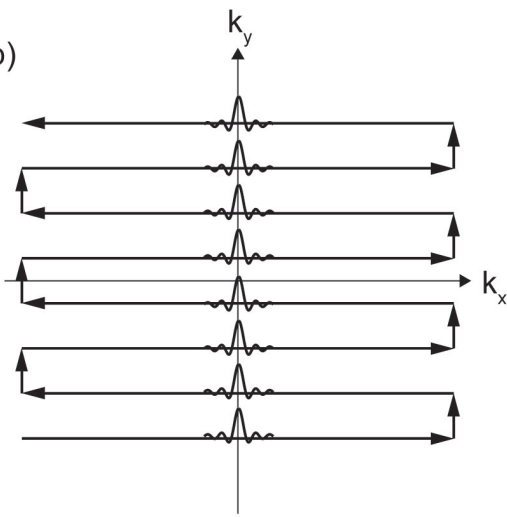

Figure 2.3: a) The echo planar imaging (EPI) readout and b) its corresponding $k$-space trajectory. Each echo is centered in $k$-space (adapted from Le Bihan et al. (2006)) 


\subsubsection{Distortions and artefacts caused by the presence of eddy currents}

The main source of distortions and artefacts in DW imaging originates from the strong gradients applied during both the encoding and readout part of the sequence. Due to the rapidly changing magnetic field during the ramping up and down of the gradients, small currents are induced in the surrounding conductors, which in turn create local magnetic fields. These magnetic fields will add up to the other gradients, modifying their shape, as illustrated in Figure 2.4(a).

(a)

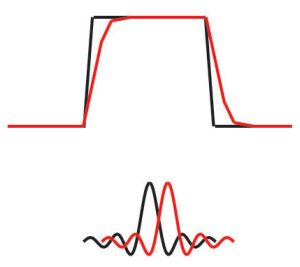

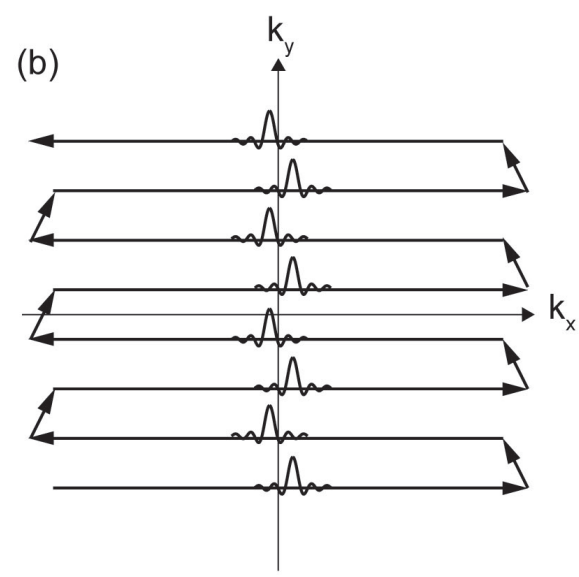

Figure 2.4: The effect of eddy currents with EPI. a) Effect of eddy currents on the gradient shape. The theoretical gradient is shown in black and the distorted gradient in red. b) k-space trajectory with the presence of eddy currents: echoes are not centered anymore (adapted from Le Bihan et al. (2006) with permissions (Copyright (C) 2006 John Wiley 83 Sons, Ltd.))

On one hand, the modified gradient shape of EPI readout gradients results in misalignment between even and odd echoes, as shown in Figure 2.4(b). The resulting image will be duplicated and a second image, called Nyquist ghost, will be superposed with a translation of half the FOV.

On the second hand, the modified gradient shape of diffusion encoding gradients results in distortions. In order to reach high $b$-value, strong and long encoding gradients are necessary. The magnetic field gradient generated by the eddy currents during the ramp up and down of the diffusion gradients may persist during the EPI readout. As a consequence, the k-space trajectory will be modified and the resulting image will be distorted (Jezzard et al., 1998). Three kind of distortions can be easily identified: translation and shear along the phase-encoding direction and a scaling effect. In order 
to reduce these effects, several methods have been proposed. By slightly increasing the currents of the gradient slopes, it is possible to compensate for eddy currents and preserve the gradient shape: this method is called premphasis and is usually implemented by the manufacturer. However, there is always some remaining currents. Reese et al. (Reese et al., 2003) proposed a double-refocused spin-echo sequence which reduces drastically eddy current distortions. This is nowadays the most popular sequence, illustrated in Figure 2.5 and used throughout this work. By using two refocusing pulses and splitting diffusion gradients, produced eddy currents tend to cancel each other and distortions are considerably reduced. In addition, post-processing method have also been suggested to get rid of remaining distortions. These methods are often combined with motion correction by proper realignment of the data (Jezzard et al., 1998), (Mohammadi et al., 2010).

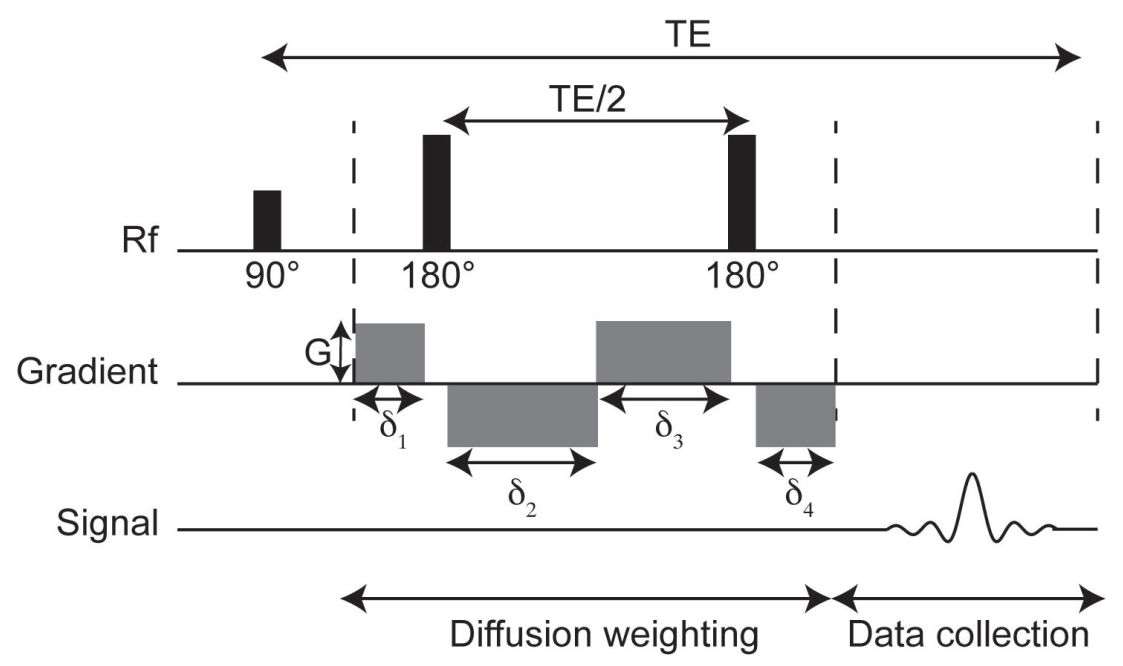

Figure 2.5: The double refocused spin echo sequence. Use of negative gradients reduces the effects of eddy currents

\subsubsection{Other artefacts}

Diffusion MRI is sensitive to a number of other artefacts and pitfalls which have been described in details in some reviews and books (Johansen-Berg and Behrens, 2009, Jones, 2004, Le Bihan et al., 2006). Here is a brief overview of the main sources of artefacts. 


\section{EPI artefacts}

Apart from eddy current induced artefacts, single-shot spin-echo EPI is wellknown to be sensitive to $B_{0}$ field inhomogeneity. Due of the low bandwidth in the phase-encoding direction, $\mathrm{B}_{0}$ field inhomogeneity can cause considerable distortions along that direction (usually anterior-posterior). Depending on the sign of the phase-encoding blips in k-space during the EPI readout, the resulting images are either squeezed or stretched. These distortions do not have an effect on the derived diffusion tensors, as they are not dependent on the diffusion gradients. However, images are not anatomically correct, which makes difficult the matching between diffusion and structural images and the data normalization. A common solution is to acquire a $\mathrm{B}_{0}$ field map and unwarp the image through a phase correction (Jezzard and Balaban. 1995). Low phase-encoding bandwidth makes EPI acquisition also sensitive to chemical shifts, in particular the fat/water one. A fat saturation pulse is systematically used to keep only the signal of interest, water in the case of diffusion imaging.

\section{Motion artefacts}

In DW imaging, as in many other imaging techniques, motion between volumes can be corrected via registration methods and it will be the object of discussion in chapter 3 . However, if the patient moves during a volume acquisition, it is more difficult to correct and it can cause strong artefact. When global motion occurs during the diffusion encoding gradients, additional motion appears at the molecular level. These movements create very large phase shifts, leading to a global drop out of the signal in the slice, as illustrated in Figure 2.6. This kind of artefact is better seen on sagittal view rather than on the axial one: usually only few slices are corrupted, showing a "zebra pattern", as they are usually acquired in an interleaved fashion.

\section{Cardiac pulsation and respiration}

Another potential source of bias in diffusion imaging is the presence of noise induced by cardiac pulsation and respiration. The main effect of cardiac pulsation is a huge signal dropout in certain regions like close to the ventricles (Nunes et al. 2005). As modeling this type of noise and thus correcting for it a posteriori is very challenging, practical methods like cardiac gating have been shown to give better results (Chung et al., 2010, Nunes et al. 2005). Another solution is the application of robust statistics technique such as RESTORE (Chang et al., 2005). 


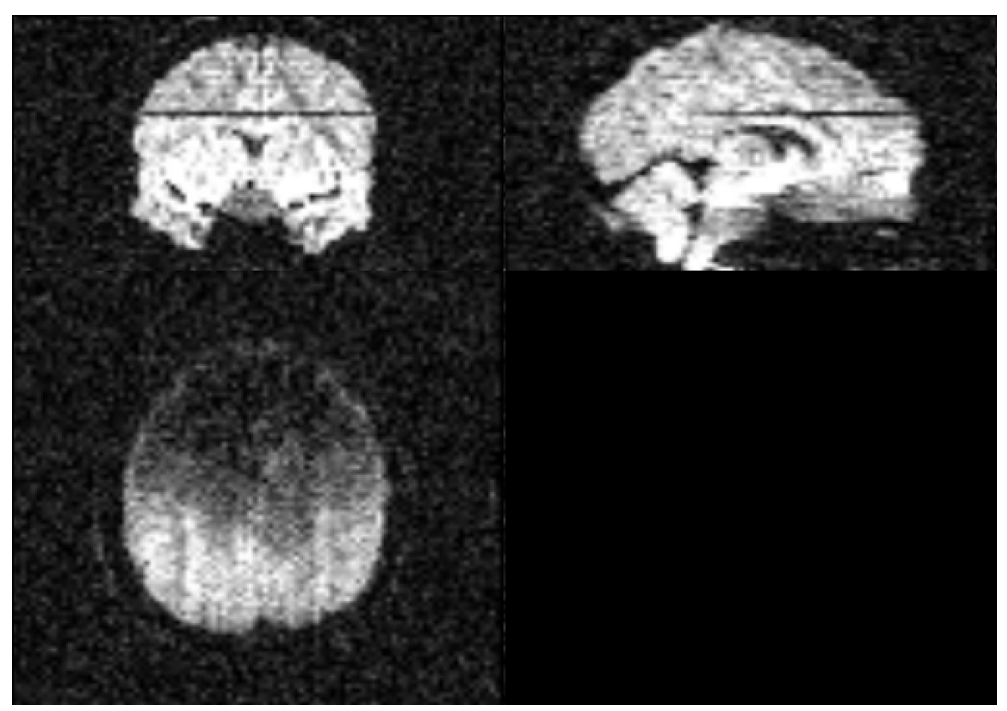

Figure 2.6: Example of a volume corrupted by motion

\subsection{Data modelling}

\subsubsection{Diffusion weighted imaging}

Diffusion weighted imaging aims to find out the molecular displacement in one particular spatial direction by resolving Equation 2.8. However the signal observed in one voxel results from the integration, on a statistical basis, of all the microscopic displacement distributions of the water molecules present in this voxel. Therefore, it has been suggested to replace the intrinsic diffusion coefficient $D$ by an apparent diffusion coefficient $A D C$ (Le Bihan et al. 1986). In clinics, $A D C$ has been shown to be relevant for the diagnostic of ischemic stroke (Baird and Warach, 1998) and this is the most established clinical application of diffusion imaging. However, this model does not take into account diffusion anisotropy, which makes its use quite limited.

\subsubsection{Diffusion tensor imaging}

\section{Diffusion tensor estimation}

Diffusion tensor imaging (DTI) was first introduced by Basser et al. (Basser et al., 1994). It is a good framework to study diffusion anisotropy in the case of the Gaussian approximation. In order to assess the anisotropy which is present in brain tissues, diffusion is no longer characterized by a single diffusion coefficient $D$, but by a second rank symmetrical tensor, called 
diffusion tensor $\mathbf{D}$ :

$$
\mathbf{D}=\left[\begin{array}{lll}
D_{x x} & D_{x y} & D_{x z} \\
D_{x y} & D_{y y} & D_{y z} \\
D_{x z} & D_{y z} & D_{z z}
\end{array}\right]
$$

Diagonal elements represent diffusion in the direction of the axis and offdiagonal elements correspond to the correlation of the movements of molecules between these axis. Similarly, the $b$-value becomes a $3 \times 3$ symmetrical matrix, which is defined for each DW image for different shapes of the field gradient pulse $\mathbf{g}$ and the $b$-value. Analytical expressions for $b$-matrix can be found in Mattiello et al. (1997). The $b$-matrix is written as follow:

$$
\mathbf{B}=\left[\begin{array}{ccc}
b_{x x} & b_{x y} & b_{x z} \\
b_{x y} & b_{y y} & b_{y z} \\
b_{x z} & b_{y z} & b_{z z}
\end{array}\right]=b\left[\begin{array}{ccc}
g_{x}^{2} & g_{x} g_{y} & g_{x} g_{z} \\
g_{x} g_{y} & g_{y}^{2} & g_{y} g_{z} \\
g_{x} g_{z} & g_{y} g_{z} & g_{z}^{2}
\end{array}\right]
$$

Then, the signal attenuation becomes:

$$
\frac{S}{S_{0}}=\exp \left(-\sum_{i=x, y, z} \sum_{j=x, y, z} b_{i j} D i j\right)=\exp \left(-b \mathbf{g} \mathbf{D} \mathbf{g}^{\prime}\right)
$$

where $S$ is the signal acquired at $b \neq 0$ and $S_{0}$ the signal acquired for $b=0$. As the diffusion tensor is symmetric, a typical DTI acquisition requires the acquisition of DW images in 6 non-collinear directions and an additional non-DW image. The number of directions has been a vast object of investigation, see Jones et al. (1999), Skare et al. (2000), Papadakis et al. (2000), Hasan et al. (2001), Jones and Basser (2004), Ni et al. (2006). In general, a set of 30 directions, uniformely distributed on the $3 \mathrm{D}$ sphere is recommended. The set of gradient directions determined by Jones et al. (Jones et al. (1999) by using an analogy with electrostatic repulsion, is usually implemented in standard diffusion sequences.

Concerning the estimation of the tensor itself, the most popular method due to its low computational time, is the linear least-squares estimation (Basser et al. 1994). Assuming a set of $N$ measurements, the problem can be summarized by the following expression:

$$
\mathbf{X}=\mathbf{b d}
$$

where $\mathbf{X}$ is a vector containing the $N$ log-transformed signal intensities:

$$
\mathbf{X}=\left[\begin{array}{llll}
\log \left(S_{1} / S_{0}\right) & \log \left(S_{2} / S_{0}\right) & \ldots & \log \left(S_{N} / S_{0}\right)
\end{array}\right]^{T}
$$


B is a $N \times 6$ matrix containing the elements of the $b$-matrix for each measurements:

$$
\mathbf{b}=\left[\begin{array}{cccccc}
b_{1 x x} & 2 b_{1 x y} & 2 b_{1 x z} & b_{1 y y} & 2 b_{1 y z} & b_{1 z z} \\
b_{2 x x} & 2 b_{2 x y} & 2 b_{2 x z} & b_{2 y y} & 2 b_{2 y z} & b_{2 z z} \\
\cdot & \cdot & \cdot & \cdot & \cdot & \cdot \\
\cdot & \cdot & \cdot & \cdot & \cdot & \cdot \\
\cdot & \cdot & \cdot & \cdot & \cdot & \cdot \\
b_{N x x} & 2 b_{N x y} & 2 b_{N x z} & b_{N y y} & 2 b_{N y z} & b_{N z z}
\end{array}\right]
$$

and $\mathbf{d}$ is a vector containing the diffusion tensor elements:

$$
\mathbf{d}=\left[D_{x x} D_{x y} D_{x z} D_{y y} D_{y z} D_{z z}\right]^{T}
$$

If $N=6$, the equation is simply solved by taking the inverse:

$$
\mathbf{d}=\mathbf{b}^{-1} \mathbf{X}
$$

If $N>6$, the tensor is estimated by computing the pseudo-inverse of $\mathbf{b}$ :

$$
\mathbf{d}=\left(\mathbf{b}^{T} \mathbf{b}\right)^{-1} \mathbf{b}^{T} \mathbf{X}
$$

The main drawback of this approach is that one estimates a symmetrical matrix but there is no guarantee that it is a physically meaningful diffusion tensor, i.e. that it is a definite semi-positive matrix. This constraint can be violated by the presence of noise. More robust methods such as Cholesky decomposition can be used in order to get positive definite tensors (Koay 2011). In addition, more complex method like RESTORE (Chang et al. 2005) have been suggested in order to get more reliable diffusion estimates, less influenced by both thermal and physiological noise (for example, by detecting outliers).

\section{Diffusion tensor representation}

The diffusion tensor is usually diagonalized using eigenvalue decomposition and is defined by:

$$
\mathbf{D}=V \cdot E \cdot V^{-1}
$$

where $V$ is the matrix of eigenvectors, representing the new basis and $E$ is the diagonal matrix of eigenvalues $\lambda_{1}, \lambda_{2}, \lambda_{3}$, representing the diffusion in the frame of the eigenvectors:

$$
E=\left[\begin{array}{ccc}
\lambda_{1} & 0 & 0 \\
0 & \lambda_{2} & 0 \\
0 & 0 & \lambda_{3}
\end{array}\right]
$$




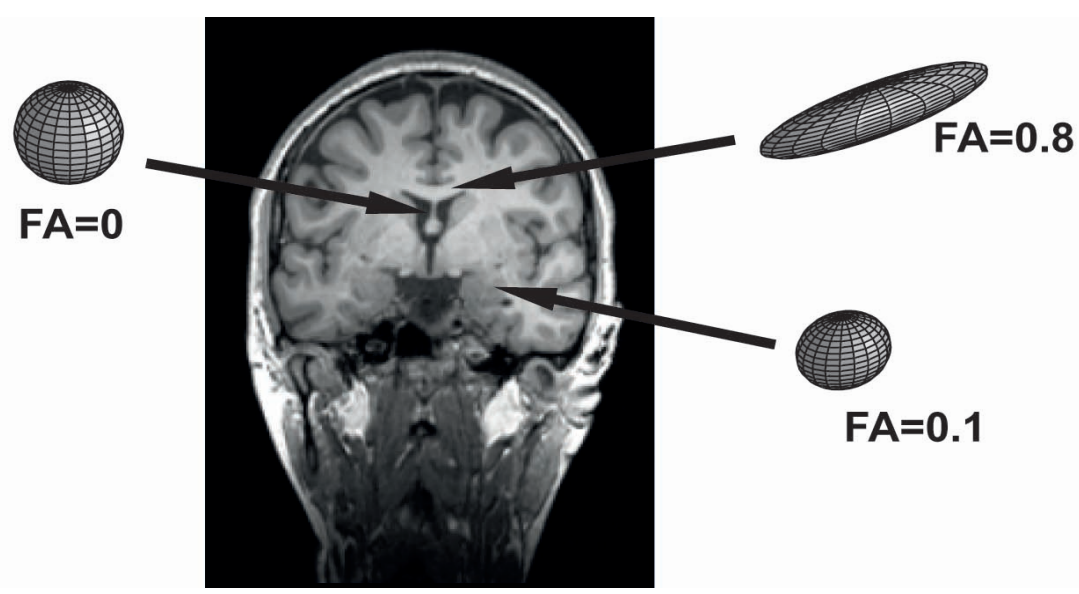

Figure 2.7: Typical diffusion tensor shapes and FA values found in the three kind of brain tissue

In this new framework, the tensor can be represented by an ellipsoid as illustrated in Figure 2.7. The three axes of the ellipsoid are given by the three eigenvalues and they are oriented along each of the three eigenvectors. This ellipsoid is a representation of the diffusion distance covered in space by molecules in a given diffusion time. Isotropic diffusion is represented by a sphere, and anisotropic diffusion by an elongated cigar-shaped ellipsoid. Several rotationally invariant parameters can be derived from the diffusion tensor, such as the mean diffusivity (MD), giving the overall size of the ellipsoid, and the fractional anisotropy (FA), related to the degree of anisotropy of the tensor (Basser, 1995). MD and FA are defined by the following expressions:

$$
\begin{gathered}
M D=\frac{\lambda_{1}+\lambda_{2}+\lambda_{3}}{3} \\
F A=\sqrt{\frac{3}{2}} \sqrt{\frac{\left(\lambda_{1}-\bar{\lambda}\right)^{2}+\left(\lambda_{2}-\bar{\lambda}\right)^{2}+\left(\lambda_{3}-\bar{\lambda}\right)^{2}}{\lambda_{1}^{2}+\lambda_{2}^{2}+\lambda_{3}^{2}}}
\end{gathered}
$$

FA ranges from 0 to 1 . A FA value of zero represents an isotropic medium while a value of 1 represents a unidirectional anisotropy. In WM, FA is closer to 1, demonstrating a strong directionality of the diffusion along the fiber, as shown in Figure 2.7. As illustrated in Figure 2.7, in GM and CSF, diffusion is isotropic (FA ranges from 0 to 0.2 ), meaning molecules have the same probability to diffuse in all directions but MD is much higher in $\mathrm{CSF}$, as the water molecules are less restricted than in GM, meaning that molecules travel in a lower averaged distance. 


\subsubsection{Diffusion kurtosis imaging}

\section{Signal representation}

DTI is built under the assumption that the probability distribution function (PDF) of the displacement of water molecules in the brain is Gaussian. In turn, this predicts a monoexponential attenuation of the diffusion-weighted signal. This assumption is approximately valid for a range of $b$-values up to $1000 \mathrm{~s} / \mathrm{mm}^{2}$. Above this value, the signal decay deviates from the monoexponential and cannot be fitted by Equation 2.8. The PDF is no longer precisely Gaussian, and the diffusion is referred as non-Gaussian. The deviation of a random variable distribution from the Gaussian distribution can be quantified by a dimensionless metric called excess kurtosis, defined by (Jensen et al., 2005, Jensen and Helpern, 2010):

$$
K=\frac{M_{4}}{M_{2}^{2}}-3
$$

where $\mathrm{M}_{n}$ is the $n^{\text {th }}$ moment of a distribution about its mean value. The excess kurtosis is null if the distribution is Gaussian, positive if the distribution is sharper (more weight in the center) and negative when the distribution is flatter as illustrated in Figure 2.8, with three examples of displacement probability functions.

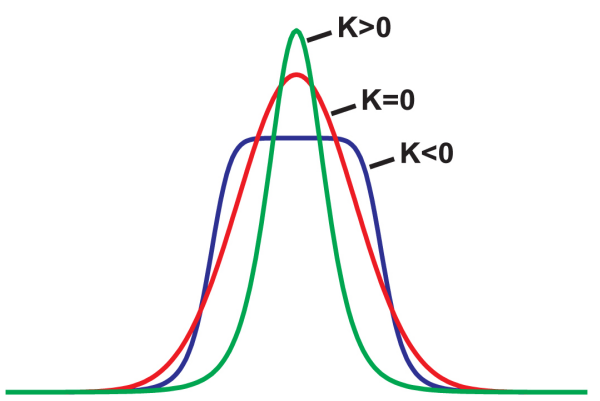

Figure 2.8: Examples of distributions with different excess kurtosis. The red one $(K=0)$ corresponds to a Gaussian distribution.

Based on this metric, diffusion kurtosis imaging (DKI) has been introduced by (Jensen et al., 2005) in order to quantify the effects of the non-Gaussian diffusion propagator in the diffusion-weighted signal. As for DTI, this is a model-free approach, which means there is no assumption about the underlying tissue structure. In that case, the cumulant expansion of the DW signal is taken up to second order on the strength of the diffusion weighting ( $b$-value), and the signal decay $\log \left(S / S_{0}\right)$ is approximated by (Jensen et al. 
2005, Jensen and Helpern, 2010):

$$
\ln \left(\frac{S}{S_{0}}\right) \simeq-b D_{a p p}+\frac{1}{6} b^{2} D_{a p p}^{2} K_{a p p}
$$

where $D_{a p p}$ is the apparent diffusion coefficient, $K_{a p p}$ the apparent kurtosis, and $b$ is the $b$-value, as described in the section 2.4.2. Compared to DTI, DKI requires the acquisition of an additional series of DW images at higher $b$-value, usually in the range of $2000-3000 \mathrm{~s} / \mathrm{mm}^{2}$. The upper range is constrained by the following equation, which ensures a constant negative decay of the fit:

$$
b \leq \frac{3}{D_{a p p} K_{a p p}}
$$

\section{Diffusion kurtosis tensor estimation}

Similar to the $3 \times 3$ elements of the diffusion tensor, one can define a kurtosis tensor $\mathbf{W}$. It is a $3 \times 3 \times 3 \times 3$ elements tensor of the $4^{\text {th }}$ order. The diffusion and kurtosis coefficients, for a given direction $\mathbf{g}$, are defined by:

$$
\begin{gathered}
D(\mathbf{g})=\sum_{i, j=1}^{3} g_{i} g_{j} D_{i j} \\
K(\mathbf{g})=\frac{M D^{2}}{D(\mathbf{g})^{2}} \sum_{i, j, k, l=1}^{3} g_{i} g_{j} g_{k} g_{l} W_{i j k l}
\end{gathered}
$$

where $D_{i j}$ represents the diffusion tensor elements and $W_{i j k l}$ the kurtosis tensor elements.

Equation 2.24 is fitted to the data to estimate $D_{a p p}$ and $K_{a p p}$. Similar to DTI, two linear systems based on equations 2.26 and 2.27 are then solved to get both the diffusion and the kurtosis tensors (Lu et al., 2006). As the diffusion tensor, the kurtosis tensor is symmetrical, meaning that measurements along at least 15 non-collinear directions are required to be able to estimate it.

\section{Representation and metrics}

In order to make the interpretation of the parameters easier, the kurtosis tensor $\mathbf{W}$ is usually projected into the Cartesian frame of reference defined by the eigenvectors of the corresponding diffusion tensor $\mathbf{D}$ : this tensor will be written $\mathbf{W}$.

Mean kurtosis (MK) is defined as the averaged $K_{a p p}$ over all directions and 
is formally written as the surface integral (Jensen and Helpern, 2010):

$$
M K=\frac{1}{4 \pi} \int d \Omega_{\mathbf{g}} K(\mathbf{g})
$$

where $d \Omega_{\mathrm{g}}$ represents the solid angle element for the direction $\mathbf{g}$. This integral can be analytically described by:

$$
\begin{aligned}
M K & =F_{1}\left(\lambda_{1}, \lambda_{2}, \lambda_{3}\right) \tilde{W}_{1111}+F_{1}\left(\lambda_{2}, \lambda_{1}, \lambda_{3}\right) \tilde{W}_{2222}+F_{1}\left(\lambda_{3}, \lambda_{2}, \lambda_{1}\right) \tilde{W}_{3333} \\
& +F_{2}\left(\lambda_{1}, \lambda_{2}, \lambda_{3}\right) \tilde{W}_{2233}+F_{2}\left(\lambda_{2}, \lambda_{1}, \lambda_{3}\right) \tilde{W}_{1133}+F_{2}\left(\lambda_{3}, \lambda_{2}, \lambda_{1}\right) \tilde{W}_{1122}
\end{aligned}
$$

where

$$
\begin{array}{r}
F_{1}\left(\lambda_{1}, \lambda_{2}, \lambda_{3}\right)=\frac{\left(\lambda_{1}+\lambda_{2}+\lambda_{3}\right)^{2}}{18\left(\lambda_{1}-\lambda_{2}\right)\left(\lambda_{1}-\lambda_{3}\right)} \\
{\left[\frac{\sqrt{\lambda_{2} \lambda_{3}}}{\lambda_{1}} R_{F}\left(\frac{\lambda_{1}}{\lambda_{2}}, \frac{\lambda_{1}}{\lambda_{3}}, 1\right)+\frac{3 \lambda_{1}^{2}-\lambda_{1} \lambda_{2}-\lambda_{2} \lambda_{3}-\lambda_{1} \lambda_{3}}{3 \lambda_{1} \sqrt{\lambda_{2} \lambda_{3}}} R_{D}\left(\frac{\lambda_{1}}{\lambda_{2}}, \frac{\lambda_{1}}{\lambda_{3}}, 1\right)-1\right]}
\end{array}
$$

and

$$
\begin{array}{r}
F_{2}\left(\lambda_{1}, \lambda_{2}, \lambda_{3}\right)=\frac{\left(\lambda_{1}+\lambda_{2}+\lambda_{3}\right)^{2}}{3\left(\lambda_{2}-\lambda_{3}\right)^{2}} \\
{\left[\frac{\lambda_{2}+\lambda_{3}}{\sqrt{\lambda_{2} \lambda_{3}}} R_{F}\left(\frac{\lambda_{1}}{\lambda_{2}}, \frac{\lambda_{1}}{\lambda_{3}}, 1\right)+\frac{2 \lambda_{1}-\lambda_{2}-\lambda_{3}}{3 \sqrt{\lambda_{2} \lambda_{3}}} R_{D}\left(\frac{\lambda_{1}}{\lambda_{2}}, \frac{\lambda_{1}}{\lambda_{3}}, 1\right)-2\right]}
\end{array}
$$

$R_{F}$ and $R_{D}$ represent Carlson's elliptic integrals (Carlson, 1979). Axial kurtosis $(\mathrm{AK})$ is defined as:

$$
A K=\frac{\left(\lambda_{1}+\lambda_{2}+\lambda_{3}\right)^{2}}{9 \lambda_{1}^{2}} \bar{W}_{1111}
$$

and radial kurtosis $(\mathrm{RK})$ :

$$
R K=G_{1}\left(\lambda_{1}, \lambda_{2}, \lambda_{3}\right) \bar{W}_{2222}+G_{1}\left(\lambda_{1}, \lambda_{3}, \lambda_{2}\right) \bar{W}_{3333}+G_{1}\left(\lambda_{1}, \lambda_{2}, \lambda_{3}\right) \bar{W}_{2233}
$$

where

$$
G_{1}\left(\lambda_{1}, \lambda_{2}, \lambda_{3}\right)=\frac{\left(\lambda_{1}+\lambda_{2}+\lambda_{3}\right)^{2}}{18 \lambda_{2}\left(\lambda_{2}-\lambda_{3}\right)^{2}}\left(2 \lambda_{2}+\frac{\lambda_{3}^{2}-3 \lambda_{2} \lambda_{3}}{\sqrt{\lambda_{2} \lambda_{3}}}\right)
$$

and

$$
G_{2}\left(\lambda_{1}, \lambda_{2}, \lambda_{3}\right)=\frac{\left(\lambda_{1}+\lambda_{2}+\lambda_{3}\right)^{2}}{3\left(\lambda_{2}-\lambda_{3}\right)^{2}}\left(\frac{\lambda_{2}+\lambda_{3}}{\sqrt{\lambda_{2} \lambda_{3}}-2}\right)
$$




\section{Why using kurtosis ?}

When using DKI, the conventional DTI parameters such as FA and MD are estimated as well as MK. Kurtosis allows one to quantify the degree of non-Gaussianity and provides additional information about the level of organization and the heterogeneity of WM tissue (Jensen and Helpern, 2010). Within the frame of the WM model, kurtosis metrics were related to the axonal water fraction (Fieremans et al., 2011) and the restricted volume fraction using the CHARMED model (De Santis et al., 2011). Furthermore, as MK does not require anisotropic tissue organization, GM microstructure can also be investigated (Jensen and Helpern, 2010, Helpern et al., 2011). In recent years, the interest for DKI has been continuously growing and MK has shown great potential as a biomarker to detect tissue abnormalities, being more sensitive to changes than classical DTI metrics. Promising results have been reported in the study of ischemic stroke in both human (Hui et al., 2008) and animal models (Grinberg et al., 2012), brain gliomas (Van Cauter et al., 2012) and epilepsy (Gao et al., 2012). DKI might thus become a useful clinical tool in the coming years.

\subsubsection{Other Non-Gaussian approches}

\section{Biexponential model}

Biexponential model was one of the first attempt to overcome DTI restriction about free diffusion. This model is based on two compartments both with Gaussian diffusion, but with different diffusion coefficient, referred as slow and fast diffusion. The signal decay is then described as follows (Niendorf et al., 1996):

$$
S=f_{f} \exp \left(-b D_{f}\right)+\left(1-f_{f}\right) \exp \left(-b D_{s}\right)
$$

where $f_{f}$ is the relative fraction of the fast component, $D_{f}$ and $D_{s}$ are the diffusion coefficients of the fast and the slow component.

\section{Structural Connectivity}

The study of structural connectivity using diffusion MRI is often referred as tractography. Basic tractography using DTI consists in following the main diffusion direction (represented by the first eigenvector) which is considered to be the direction of the underlying fiber (Jones et al., 1999, Mori et al. 1999, Conturo et al., 1999). However this method has shown strong limitations, especially to resolve regions of crossing fibers. More recent methods have been developed to overcome this problem. Amongst others, one can mention q-ball imaging (Tuch, 2004) or diffusion spectrum imaging (Wedeen 
et al. 2008). The main advantage of these methods is to fit the data directly without assuming a particular distribution. 


\section{Chapter 3}

\section{Motion correction for diffusion experiments}

\subsection{Introduction}

Diffusion MRI is a popular method to study brain structure and relates it to brain function. Recently, more advanced models, mainly for brain tractography have been proposed to get more complex details and detect smaller changes. For that purpose, acquisition has been pushed towards higher $b$-values and more diffusion directions, in order to resolve diffusion profiles with improved angular accuracy. This increases the acquisition time significantly. While a standard DTI acquisition can be done in few minutes, adding $\mathbf{b}$-values or several diffusion directions can increase the acquisition time to 15 minutes or more. During this long time, it is certainly unlikely that subjects stay still. Correcting for the effect of motion is thus an important step in the processing of diffusion data. The standard method consists simply in the coregistration of DW volumes to the first non-DW volume (Rohde et al., 2004). However, this step is not as trivial as it seems: DW images contain orientation information, and thus each image has its own intensity profile, which does not match the intensity in non-DW images. Moreover, with the use of higher $b$-values, the presence of noise increases the mismatch between images, as the contrast between tissues is very low and thus extremely different from the non-DW contrast.

In this introduction, we will briefly describe basic registration methods. In a second part, we will review the different methods used in diffusion MRI for the intra-subject registration, for motion and distortion correction. 


\subsubsection{Coregistration in MRI}

Registration methods have been reviewed previously in (Brown, 1992, Zitova and Flusser, 2003, Bai, 2009). They rely on three components (Crum et al., 2004):

- Transformation model: how images are transformed to match the source image, which parameters are considered.

- Similarity measure: how well two images match.

- Optimization process: how parameters of the transformation model are estimated to maximize the matching criteria.

\section{Transformation}

To match image $I_{1}$ to image $I_{2}$, several types of transformations can be considered. A transformation is a set of parameters and geometrical operations which brings one object into the space of the second object. These transformations can be rigid-body (shape and size are preserved), affine or non-linear. The latter is not considerer in this work.

- Affine transformation

An affine transformation is described by:

$$
T(v)=R M X v+t
$$

where $\mathrm{v}$ is the location vector in $3 \mathrm{D}$ space, $\mathrm{R}$ is the rotation matrix, $\mathrm{t}$ is the translation vector, $\mathrm{M}$ is the scaling matrix and $\mathrm{X}$ the shearing matrix. The rotation matrix is the matrix product of the rotations around each axis:

$$
\begin{array}{r}
R=\left[\begin{array}{ccc}
1 & 0 & 0 \\
0 & \cos \left(r_{x}\right) & \sin \left(r_{x}\right) \\
0 & -\sin \left(r_{x}\right) & \cos \left(r_{x}\right.
\end{array}\right] \\
{\left[\begin{array}{ccc}
\cos \left(r_{y}\right) & 0 & \sin \left(r_{y}\right) \\
0 & 1 & 0 \\
-\sin \left(r_{y}\right) & 0 & \cos \left(r_{y}\right)
\end{array}\right]} \\
\\
{\left[\begin{array}{cccc}
\cos \left(r_{z}\right) & \sin \left(r_{z}\right) & 0 \\
-\sin \left(r_{z}\right) & \cos \left(r_{z}\right) & 0 \\
0 & 0 & 1
\end{array}\right]}
\end{array}
$$

where $r_{x}, r_{y}, r_{z}$ are the rotations in radians. The rotation around $\mathrm{x}-$ axis is called pitch, around the y-axis roll and around the z-axis yaw. The scaling matrix is written as:

$$
M=\left[\begin{array}{ccc}
m_{x} & 0 & 0 \\
0 & m_{y} & 0 \\
0 & 0 & m_{z}
\end{array}\right]
$$


and the shearing matrix:

$$
X=\left[\begin{array}{ccc}
1 & s_{3} & s_{2} \\
0 & 1 & s_{1} \\
0 & 0 & 1
\end{array}\right]
$$

\section{- Rigid-body transformation}

A rigid-body transformation $\mathrm{T}$ is a particular case of affine transformation where shearing is set to zero and scaling to 1 . It has only 6 parameters ( 3 translations and 3 rotations) and can be formally written like this:

$$
T(v)=R v+t
$$

For realigning brain images of the same subject, a rigid-body transformation should be used, as it preserves shape and size, which are supposed to remain the same during the acquisition. However, the presence of artefacts can cause distortions, and it that case, a full affine transformation can be used.

\section{Similarity measure}

The goal of the similarity measure, also called the cost function is to measure how well two images are aligned. Image $X$ is the reference and image $Y$ the floating image. The problem is then to maximize the similarity between $X$ and $Y$ by finding the optimal parameters of the transformation $T$ applied to $Y$. As it is usually more convenient from a mathematic point of view to minimize a function rather than maximizing it, the cost function quantifies the dissimilarity between the images and then the transformation $T^{*}$ is described by:

$$
T^{*}=\arg \min C(Y, T(X))
$$

where $\mathrm{C}$ is the cost function and $T(X)$ the image $X$ transformed by $T$. Cost functions can be either geometrically-based, using features such as landmarks, corners, curves, or intensity-based, which is the most common option.

In MRI, registration problems can be divided in two categories: intrasubject and inter-subject registration. In this chapter, we will focus only the intra-subject case. Within this category, two subcategories can be distinguished: intra-modality and inter-modality. We will briefly describe the most common cost functions for these cases, which are least squares and normalized correlation for intramodal registration, and mutual information, normalized mutual information and correlation ratio for the intermodal registration. 
- Least square

This is the most basic method. It consists in minimizing the sum of the squared difference over all corresponding voxels in two images.

$$
\left.C^{L S}=\sum_{i=1}^{N}\left(I_{1 i}-I_{2 i}\right)\right)^{2}
$$

where $\mathrm{N}$ is the total number of voxels, $I_{1 i}$ and $I_{2 i}$ the voxel intensities of image $I_{1}$ and $I_{2}$. This function assumes similar intensities in both images, thus it is suitable only for intramodal registration.

- Normalized correlation

Normalized correlation is more robust than least squares, as it is not sensitive to intensity scales, and is defined as:

$$
C^{N C}=\frac{\sum_{i=1}^{N}\left(I_{1 i} \cdot I_{2 i}\right)}{\sqrt{\sum_{i=1}^{N} I_{1 i}^{2}} \sqrt{\sum_{i=1}^{N} I_{2 i}^{2}}}
$$

It assumes a linear relationship between the intensities of the images and thus it is used only for intramodal registration.

\section{- Mutual information}

As intermodal registration is of high interest in medical imaging, more advanced cost functions have been developed. In particular, mutual information (MI) has become quite popular. This method originates from the information theory, which is a general theory about quantification of information, originally applied for storing and compressing data. It is defined as:

$$
C^{M I}=H\left(I_{1}, I_{2}\right)-H\left(I_{1}\right)-H\left(I_{2}\right)
$$

where $\mathrm{H}$ is the standard entropy, which is a measure of the complexity of the information of each images and $H\left(I_{1}, I_{2}\right)$ is the joint entropy. The method is based on the maximization of MI and was first introduced for image registration by Viola and Wells (1995). This method has been the subject of many improvements which are reviewed in $\mathrm{Zi}-$ tova and Flusser (2003). In particular, normalized mutual information has been proposed by Studholme et al. (1999):

$$
N M I=\frac{H\left(I_{1}\right)+H\left(I_{2}\right)}{H\left(I_{1}, I_{2}\right)}
$$




\section{Optimization}

The estimation of parameters is done via optimization. This step consists of determining the set of parameters, which minimizes or maximizes the cost function. This is usually done via complex optimization algorithms such as Gauss-Newton numerical minimization algorithm, gradient descent optimization method, Levenberg-Marquardt optimization method etc. For more details about these algorithms, see Bai (2009), Zitova and Flusser (2003).

\subsubsection{Coregistration of diffusion weighted images}

Coregistration of DW images is challenging for two main reasons: the large contrast difference between low and high $b$-value images and the presence of eddy-current distortions. In this chapter, we will focus only on movement corrections. However eddy-current induced distortions are also an important step and the different correction methods will be introduced briefly.

\section{Basic coregistration using mutual information cost function}

A common approach for motion correction in diffusion MRI is the coregistration of all the DW images $(b \geq 0)$ to the first non-DWI image $(b=0)$. However, the contrast between these two types of images is very different. Mutual information has been suggested as a robust cost function to solve this intermodal problem (Rohde et al. 2004$)$, and this method remains the standard.

\section{Model-based coregistration methods}

As mentioned earlier, basic coregistration methods might fail for realigning DW images properly, as each DW image contains different information, related to the orientation of the associated gradient. Andersson and Skare (2002) introduced a cost function, which minimizes the residual error when fitting data to the diffusion tensor model. In other words, the idea is to minimize the sum-of-square difference between the registered data and the one derived from the tensor. Later on, Bai and Alexander (2008), Nam and Park (2011) and Ben-Amitay et al. (2012) developed similar based-model approaches. The three methods are based on coregistration of each DW image to its corresponding simulated image from the calculated diffusion tensor or, in the case of Ben-Amitay et al. (2012) using the composite hindered and restricted model of diffusion (CHARMED) (Assaf and Basser, 2005), which is an expansion of DTI model taking into account the geometry of the tissues. 


\section{Real-time methods}

Most of the time, motion is corrected retrospectively. Real-time correction during the acquisition offers other possibilities. In the literature, several methods have been proposed, using navigator echoes (Alhamud et al., 2012, Kober et al., 2012), external optical tracking systems (Aksoy et al., 2011), or with simple online registration and reacquisition (Benner et al. 2011 ). However these methods are not standard and are more difficult to apply. The main advantage of these methods is the ability to detect and then reacquire data with strong signal dropout due to fast motion (e.g. within one volume acquisition). This type of artefacts cannot be corrected retrospectively and have to be removed from the analysis, reducing accuracy on the derived quantitative metrics.

\section{Particularity of diffusion imaging : Gradient rotation}

The diffusion direction orientation must be corrected together with the corresponding volume to keep coherence between the intensity and the diffusion direction. In other words, if after movement correction the volume was rotated, the same rotation must be applied to the gradient direction. When this step is omitted, a bias in the orientation information is introduced. Quantitative metrics such as $F A$ are not affected in a large extent but it may be a real issue for tractography studies, where the main diffusion direction is used for the fibers reconstruction (Leemans and Jones, 2009).

\section{The issue of eddy current distortions}

In diffusion MRI, long encoding gradients are necessary to reach high $b$ values as the gradient strength is quite limited on clinical scanners. Due to the rapidly changing magnetic field during the ramping up and down of the gradient, small currents are induced in the surrounding conductors, which in turn creates local magnetic fields. These magnetic fields will add up to the encoding gradient, causing distortions. If gradients are short enough, eddy currents appearing during the ramping up are compensated by those appearing during the ramping down. However, this in not the case in diffusion MRI: long encoding gradients are used in order to reach high $b$-values as the gradient strength is quite limited on clinical scanners. The use of a double spin echo sequence have considerably reduced the effect of eddy currents (Reese et al., 2003), but some residual currents might still be present. To correct for these residual distortions several methods have been proposed: the standard method is a 3D realignment using affine transformations (Rohde et al., 2004). Eddy current distortions are corrected 
through shearing and translations. However, it is advised to use a slice-byslice correction (Haselgrove and Moore, 1996), as eddy currents are not homogeneous through the volume (Jones and Cercignani, 2010).

\subsubsection{Objectives}

In this chapter, we aim to demonstrate that a classic coregistration of DW is not optimal and that iterative processes, as suggested by Nam and Park (2011) are preferable. We present a similar iterative model-based method making use of more specific tensor metrics measuring the distance between two tensors as a stopping criterion.

\subsection{Methods}

\subsubsection{Iterative model-based method}

As in the previous works of Andersson and Skare (2002), Nam and Park (2011), the model presented here is based on the DTI model. Part of this work was done by Furlan (2011). The main steps are:

- Estimation of the diffusion tensors using linear least square.

- Creation of the theoretical diffusion images from the estimated tensors for each direction.

- Coregistration of each individual raw DW images to the corresponding theoretical ones.

- Calculation of the global tensor difference between the current tensor images (from the corrected data set) and the previously calculated ones.

Once the tensor distance starts to increase or that 20 iterations are reached, the algorithm is stopped and the DW images are considered as realigned. The procedure is summarized in Figure 3.1 .

\section{Steps 1 and 2: Realignment of non-DW images and movement interpolation}

As an option, a preliminary step is applied for the realignment of nonDW images. This step can be implemented only in the case where extra non-DW images have been acquired in an interleaved fashion with the DW images (every few images). As non-DW images are identical, the usual coregistration step with LS or MI is very robust. These images are perfectly realigned with a rigid-body transformation. Movements between two nonDW images are assumed to change linearly and the interpolated movements 


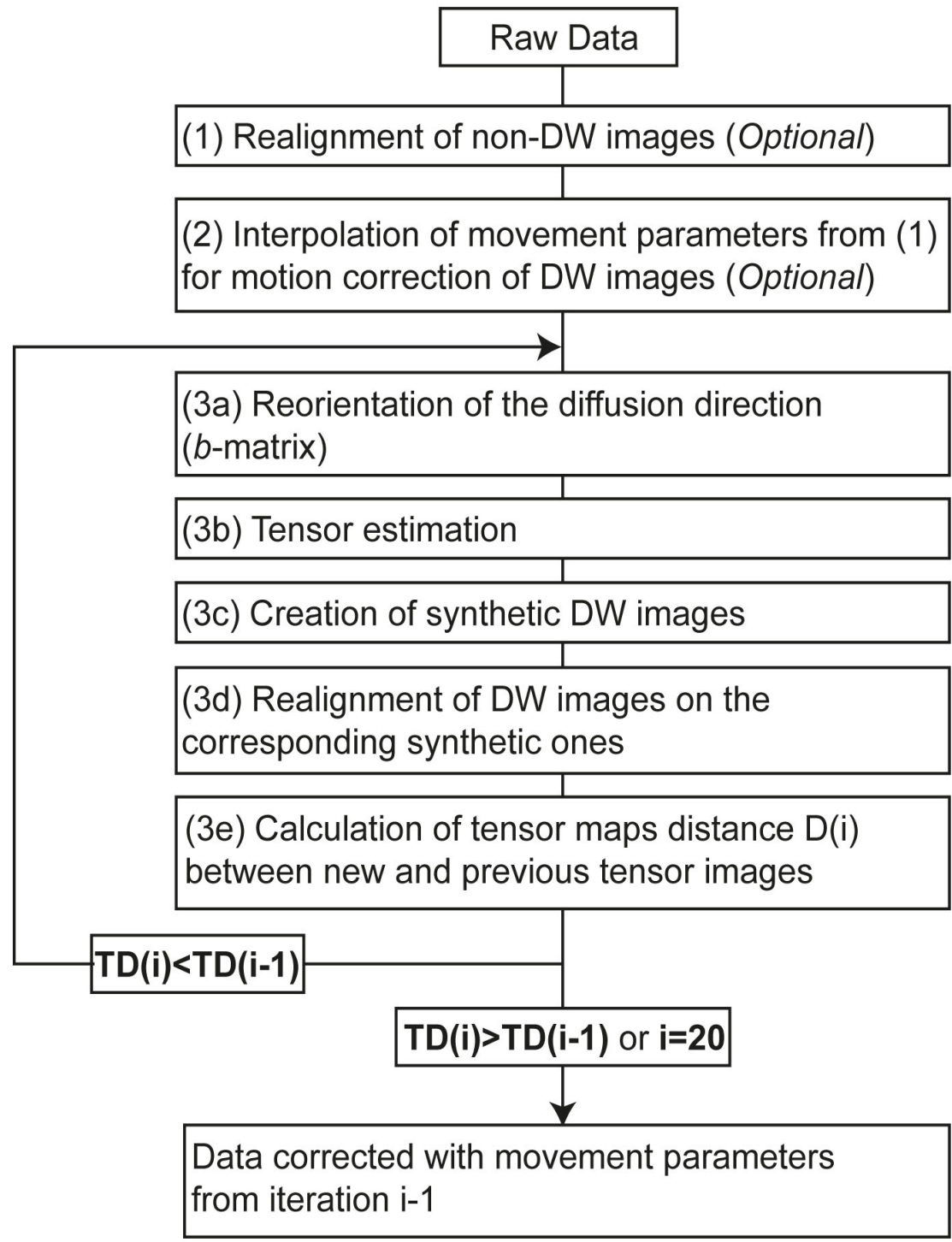

Figure 3.1: Schematic representation of the iterative procedure for realignment of diffusion weighted images

are applied to the corresponding DW images. The estimated parameters will be used to initialize the movement of the first iteration. 


\section{Step 3a: Reorientation of the diffusion directions}

As explained in the introduction, the orientation of the gradient direction is rotated according to the rotation of the image in order to keep the right orientation information.

\section{Step 3b: Tensor estimation}

The estimation of the tensor for each voxel is done via a least square estimation using the general linear model implemented in SPM8. The diffusion toolbox developed by Volkmar Glauche was used 11 .

\section{Step 3c: Synthetic DW image}

From the tensor estimation (step 3b), we get an image without diffusion weight, called $A_{0}$ and 6 diffusion tensor images, one for each parameter of the diffusion tensor (Eq. 2.10). It is then possible to create theoretical diffusion images $I_{g}$ for each diffusion direction $\mathbf{g}$, using the tensor model:

$$
I_{g}=A_{0} \exp \left(-b \mathbf{g}^{\prime} D \mathbf{g}\right)
$$

where $D$ is the diffusion tensor. $b$ is the corresponding $b$-value, in our case we used $b=1000 \mathrm{~s} / \mathrm{mm}^{2}$.

\section{Step 3d: Realignment of DW images}

Each raw image is coregistered to its paired theoretical image derived from step $3 \mathrm{c}$ with a rigid-body transformation and MI cost function. Note that the coregistration is always done on the raw images, to avoid successive resampling, which will cause smoothing in the images. The movement parameters estimated during the previous iteration (except for the first one) are used as initial parameters, to gain efficiency.

Step 3e: Calculation of the distance between two successive tensor images as a stopping criterion:

The originality of our method consists in using a tensor distance as a stopping criterion. Development of mathematical tools specific to the tensor environment is a dynamic field of research and is of particular interest for DTI. Here we applied a technique developed by Anne Collard Collard (2013), Collard et al. (2014). This novel metric is based on the spectral decomposition of the tensor, which separates the orientation information and the intensity

\footnotetext{
${ }^{1} \mathrm{http}: / /$ sourceforge.net/projects/spmtools/
} 
information. In that framework, the distance $d$, between two tensors $S_{1}$ and $S_{2}$ is defined as:

$$
d^{2}\left(S_{1}, S_{2}\right)=d^{2}\left(\Lambda_{1}, \Lambda_{2}\right)+d^{2}\left(V_{1}, V_{2}\right)
$$

where $S_{1}=V_{1} \Lambda_{1} V_{1}^{T}$ and $S_{2}=V_{2} \Lambda_{2} V_{2}^{T}, V_{1}$ and $V_{2}$ are the rotation matrices containing the eigenvectors and $\Lambda_{1}$ and $\Lambda_{2}$ are the diagonal matrices containing the eigenvalues. The eigenvalues are ordered in decreasing value in the diagonal matrices, i.e. $\lambda_{1}>\lambda_{2}>\lambda_{3}$. Eigenvectors are ordered in the same way in $V$. The distance between two tensor images containing $\mathrm{N}$ voxels is defined by:

$$
T D=\frac{1}{N} \sum_{k=1}^{N} d^{2}\left(S_{1, k}, S_{2, k}\right)
$$

where $S_{1, k}$ is the tensor of the first image at location $k$ and $S_{1, k}$ the tensor of the second image at the same location.

\subsubsection{Method assessment}

\section{Real datasets}

A standard data set was acquired on a trained and collaborative volunteer who stayed as still as possible during the acquisition. Data were acquired with a 3T head-only scanner (Magnetom Allegra, Siemens) using a twice refocused spin-echo diffusion sequence, known to significantly reduce the effect of eddy currents on image distortion (Reese et al., 2003). 60 DW images with 60 non-collinear diffusion encoding directions with a $b$-value of $1000 \mathrm{~s} / \mathrm{mm}^{2}$ were acquired together with 6 non-DW images interleaved with the DW ones. Other parameters were set to: TR/TE $=7100 / 76 \mathrm{~ms}$, matrix size : $96 \times 96 \times 64$. Voxels were $2.2 \mathrm{~mm}$ isotropic.

A second data set acquired on a Parkinson's patient was also used to observe the effect of our method compared to the standard coregistration. Data were also acquired with a $3 \mathrm{~T}$ head-only scanner (Magnetom Allegra, Siemens) using a twice refocused spin-echo diffusion sequence. 120 DW images with 120 non-collinear diffusion encoding directions with a $b$-value of $1000 \mathrm{~s} / \mathrm{mm}^{2}$ and with a $b$-value of $2500 \mathrm{~s} / \mathrm{mm}^{2}$ were acquired for a total of $240 \mathrm{DW}$ images, together with 22 non-DW images interleaved with the DW ones. As we focused on DTI model, only the first 131 images, corresponding to the acquisition at $b=1000 \mathrm{~s} / \mathrm{mm}^{2}$ were analysed. Other parameters were set to: $\mathrm{TR} / \mathrm{TE}=6800 / 91 \mathrm{~ms}$, matrix size: $88 \times 88 \times 54$. Voxels were $2.4 \mathrm{~mm}$ isotropic. 


\section{Simulated data}

In order to assess objectively the performance of the different methods, synthetic data were generated. For that purpose we used the data set acquired on a trained and collaborative volunteer described in the previous paragraph. The derived tensor images were assumed to be uncorrupted by motion and were used to generate the synthetic data set the same way as described in step $3 \mathrm{c}$ of our procedure. Realistic rigid-body movements (translations and rotations in the range of $[0-2] \mathrm{mm}$ and $[0-2]^{\circ}$ ) were generated for each DW image. These movements were applied to both the image and the gradient direction, in order to keep the orientation corresponding to each intensity profile. Two sets of data were simulated: one set with non-DW images interleaved with the DW ones and one with all the nonDW images at the beginning. These datasets allowed us to have a reference to be able to compare the different methods.

\subsubsection{Method comparison}

We compared 5 different approaches:

- Basic coregistration (BC)

- Pre-realignment of non-DW images and interpolation (Step 1 and 2 in Figure 3.1), that will refer as the interpolation method (Int)

- Iterative procedure for realignment (IPR)

- Interpolation combined with IPR (Int+IPR)

- Basic coregistration combined with IPR (Coregistration replaced steps 1 and 2 in Figure 3.1) (BC+IPR)

For all the approaches above, only rigid-body tranformations were considered.

In order to quantify the performance of each approach, several parameters were compared:

- Comparison of the estimated parameters (3 translations and 3 rotations) with the reference, as movements are known. The root mean square (RMSE) difference was calculated as the global error over $\mathrm{N}$ volumes, for each parameter $P$ :

$$
R M S E(P)=\sqrt{\sum_{i=1}^{N}\left(P_{r e f}-P_{i}\right)^{2}}
$$


and the global error on translations was defined by:

$$
R M S E\left(T_{X Y Z}\right)=\sqrt{\sum_{i=1}^{N}\left(X_{r e f}-X_{i}\right)^{2}+\left(Y_{\text {ref }}-Y_{i}\right)^{2}+\left(Z_{\text {ref }}-Z_{i}\right)^{2}}
$$

A similar error was calculated for rotations.

- Tensor distance between the reference tensors and the corrected ones as defined by Eq. 3.13 .

- FA root mean squared error defined by:

$$
F A_{\text {err }}=\sqrt{\sum_{i=1}^{N}\left(F A_{r e f}-F A_{i}\right)^{2}}
$$

where $\mathrm{N}$ is the number of voxels

- Main orientation of the fiber, defined as the angle between the reference direction $d_{r e f}$ and the corrected ones $d_{m e t h}$ :

$$
\alpha=\arccos \left(\left\langle d_{\text {ref }} \cdot d_{m e t h}\right\rangle\right)
$$

\subsection{Results}

\subsubsection{Synthetic data: non-DW images interleaved with the DW images}

\section{Estimated movements versus theoretical movements}

The rigid-body parameters characterizing the movement of the subject have been estimated with the different approaches (5 in total). Results are displayed in Figure 3.2. Theoretical movements are shown with a solid black line as a reference. The best approach is to combine an interpolation step with IPR. This is confirmed in Table 3.1, where RMSE over all volumes are reported for each parameters. Interpolation combined with IPR exhibits the best averaged scores with a value of 0.27 for the translations and a value of 0.22 for the rotations. Taken independently, interpolation performs better than IPR with a RMSE of 0.34 for translations and 0.28 for rotations. On the contrary, both approaches including a coregistration step show lower performances with RMSE values of $0.97 / 0.79$ for the translations and $1.19 / 0.87$ for the rotations. 

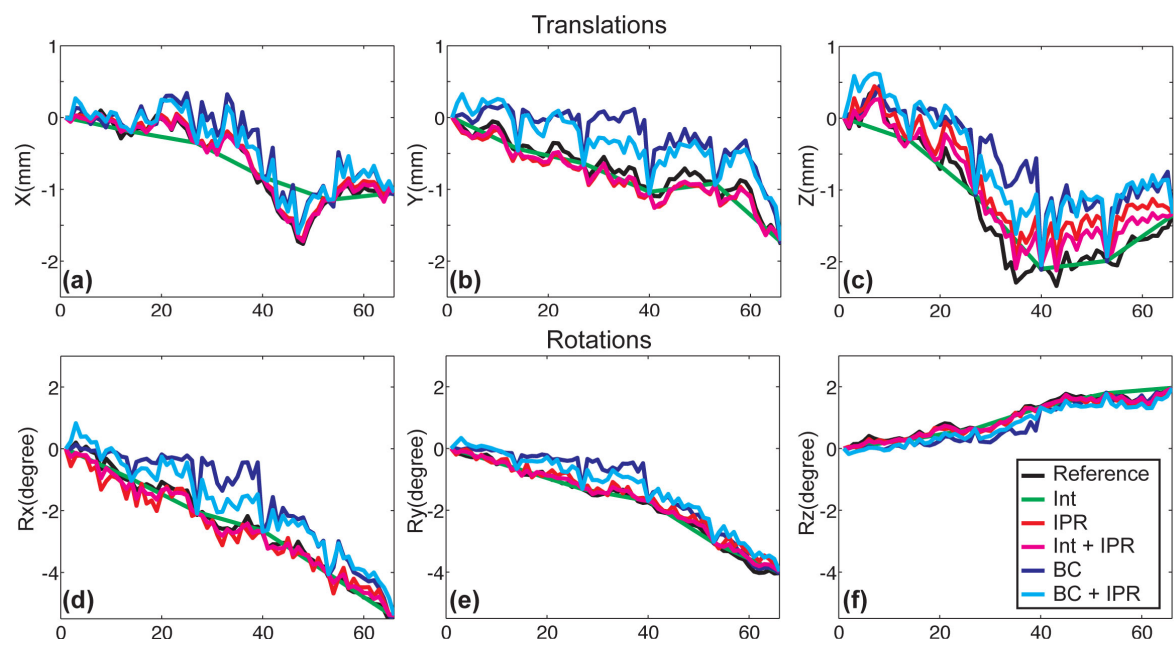

Figure 3.2: Estimated movements obtained the different approaches compared to the reference, in the case where non-DW images are interleaved with the DW images. The x-axis represents the volume number and can be seen as a time representation.

\begin{tabular}{l|cccc|cccc} 
Approach & $\mathrm{T}_{\mathrm{X}}$ & $\mathrm{T}_{\mathrm{Y}}$ & $\mathrm{T}_{\mathrm{Z}}$ & $\mathbf{T}_{\mathbf{X Y Z}}$ & $\mathrm{R}_{\mathrm{X}}$ & $\mathrm{R}_{\mathrm{Y}}$ & $\mathrm{R}_{\mathrm{Z}}$ & $\mathbf{R}_{\mathbf{X Y Z}}$ \\
\hline Int & 0.23 & 0.14 & 0.21 & $\mathbf{0 . 3 4}$ & 0.21 & 0.11 & 0.16 & $\mathbf{0 . 2 8}$ \\
IPR & 0.06 & 0.13 & 0.39 & $\mathbf{0 . 4 1}$ & 0.39 & 0.23 & 0.10 & $\mathbf{0 . 4 2}$ \\
Int + IPR & 0.04 & 0.11 & 0.24 & $\mathbf{0 . 2 7}$ & 0.18 & 0.11 & 0.06 & $\mathbf{0 . 2 2}$ \\
BC & 0.32 & 0.56 & 0.73 & $\mathbf{0 . 9 7}$ & 1.00 & 0.55 & 0.35 & $\mathbf{1 . 1 9}$ \\
BC + IPR & 0.23 & 0.38 & 0.66 & $\mathbf{0 . 7 9}$ & 0.67 & 0.49 & 0.26 & $\mathbf{0 . 8 7}$
\end{tabular}

Table 3.1: Root mean square errors calculated over all volumes for each approach and each parameter with the non-DW interleaved data set

\section{Tensor distance maps}

The tensors maps obtained with the synthetized images prior the addition of movements are considered as the reference and the distances between these tensors and those obtained with the various procedures are calculated voxel-by-voxel, to get tensor distance maps. Figure 3.3(a) shows the tensor distances if no correction is applied. It is on average quite high, 0.42 for the whole volume. After realigning data with all the different methods the tensor distance is highly reduced: down to 0.15 for Int+IPR procedure and 0.18 for BC. These differences are better highlighted when looking at specific regions of interest (ROI). The first ROI is the WM volume, defined as the area where $F A>0.2$ in the reference FA map. Two more ROIs were drawn on one slice, in regions of higher noise, according to the noisy distance map 


\begin{tabular}{l|cccc} 
Approach & Whole volume & WM volume & ROI 1 & ROI 2 \\
\hline Noisy & 0.42 & 0.62 & 2.29 & 0.19 \\
Int & 0.15 & 0.26 & 0.71 & 0.02 \\
IPR & 0.16 & 0.26 & 0.76 & 0.03 \\
Int + IPR & 0.15 & 0.26 & 0.74 & 0.02 \\
BC & 0.18 & 0.29 & 0.84 & 0.04 \\
BC + IPR & 0.18 & 0.29 & 0.83 & 0.04
\end{tabular}

Table 3.2: Mean tensor distances after and before motion correction in four different volumes of interest - Case of interleaved non-DW images

as shown in green in Figure 3.3. ROI1 is in the corpus callosum and ROI2 in a white matter area on the right part of the brain. In particular, TD is very high in the corpus callosum $(T D=2.29)$. This value goes down to 0.74 with Int+IPR procedure and 0.83 for BC. It confirms the observations made in the previous section. Interpolation and IPR procedure perform similar or lower than the combination Int+IPR except in ROI1 where TD is slightly lower (0.71 compared to 0.74). Adding IPR to BC does not improve the results.

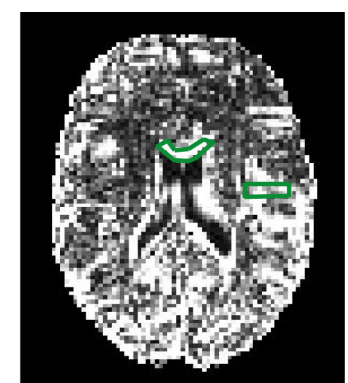

(a) Noisy VS Reference

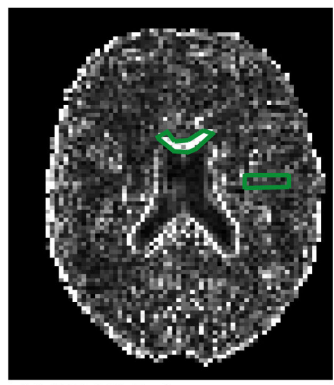

(d) IPR VS Reference

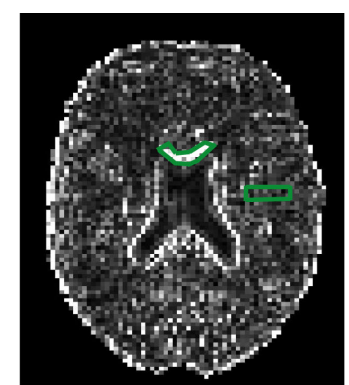

(b) Int VS Reference

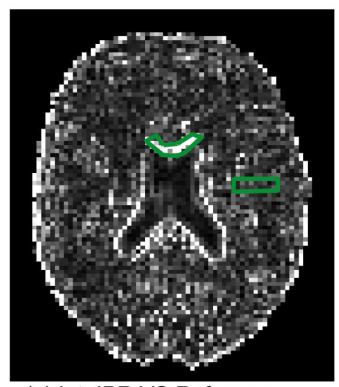

(e) Int+IPR VS Reference

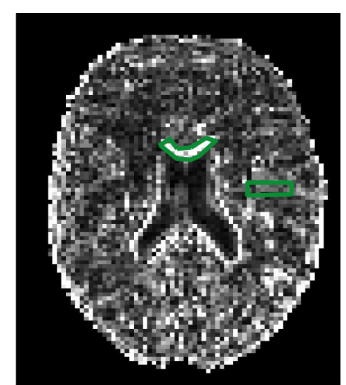

(c) BC VS Reference

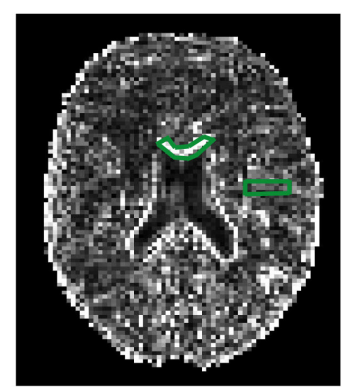

(f) BC+IPR VS Reference

Figure 3.3: Tensor distance maps for the non-DW interleaved case. 


\begin{tabular}{l|cccc} 
Approach & Whole volume & WM volume & ROI 1 & ROI 2 \\
\hline Noisy & 59.10 & 54.16 & 1.41 & 0.98 \\
Int & 36.45 & 34.58 & 0.59 & 0.36 \\
IPR & 37.19 & 34.80 & 0.65 & 0.39 \\
Int + IPR & 36.15 & 34.26 & 0.61 & 0.37 \\
BC & 43.48 & 40.24 & 0.78 & 0.52 \\
BC + IPR & 42.34 & 39.29 & 0.75 & 0.50
\end{tabular}

Table 3.3: Errors on FA after and before motion correction in four different volumes of interest - Case of interleaved non-DW images

\begin{tabular}{l|cccc} 
Approach & Whole volume & WM volume & ROI 1 & ROI 2 \\
\hline Noisy & 32.26 & 24.57 & 4.05 & 21.87 \\
Int & 14.24 & 9.55 & 3.25 & 6.13 \\
IPR & 17.52 & 11.37 & 3.04 & 7.68 \\
Int + IPR & 14.71 & 9.69 & 3.04 & 6.43 \\
BC & 23.12 & 16.00 & 3.11 & 10.32 \\
BC + IPR & 21.17 & 14.58 & 3.15 & 9.81
\end{tabular}

Table 3.4: Errors on the main fiber direction after and before motion correction in four different volumes of interest - Case of interleaved non-DW images

\section{FA and main diffusion direction}

Tensor distance embraces both the difference in anisotropy and the orientation of the diffusion. Obviously, the errors in the estimation of the tensors will propagate in the estimations of FA and the main diffusion orientation for tractography experiments. Both FA and main angle discrepancies are summarized in Tables 3.3 and 3.4. For tractography, only the orientation of the main diffusion vector is of interest, not the direction. For that reason, angles were constrained in the range of 0 to $90^{\circ}$. The effect of the correction procedure follows the same trend as for the measure of TD: Int+IPR performs the best and $\mathrm{BC}$ the worse. Adding IPR to BC slightly improves the results: $F A_{\text {err }}=42.34$ compared to $F A_{\text {err }}=43.48$ for the whole brain volume and $\alpha=21.17^{\circ}$ instead of $\alpha=23.12^{\circ}$. However it is not as good as using Int+IPR, where $F A_{\text {err }}=36.15$ and $\alpha=14.71^{\circ}$. Interestingly, in ROI1 (the corpus callosum), the error in the main direction is very low, even without any correction $\left(\alpha=4.05^{\circ}\right)$. However the difference in term of FA is much more significant: 1.41 without motion correction, 0.78 for $\mathrm{BC}$ and 0.61 for Int+IPR. These differences were well described by TD. Inversely in the second ROI, the difference in term of TD is quite small ( 0.04 for $\mathrm{BC}$ and 0.02 for Int+IPR) but a bit more higher in term of FA or angle discrepancies (respectively 0.52 and $10.32^{\circ}$ for $\mathrm{BC}$ and 0.37 and $6.43^{\circ}$ for Int + IPR. 


\begin{tabular}{l|cccc|cccc} 
Approach & $\mathrm{T}_{\mathrm{X}}$ & $\mathrm{T}_{\mathrm{Y}}$ & $\mathrm{T}_{\mathrm{Z}}$ & $\mathbf{T}_{\mathbf{X Y Z}}$ & $\mathrm{R}_{\mathrm{X}}$ & $\mathrm{R}_{\mathrm{Y}}$ & $\mathrm{R}_{Z}$ & $\mathbf{R}_{\mathbf{X Y Z}}$ \\
\hline IPR & 0.80 & 0.58 & 0.61 & $\mathbf{1 . 1 6}$ & 1.06 & 1.39 & 1.22 & $\mathbf{2 . 1 3}$ \\
BC & 0.32 & 0.50 & 0.53 & $\mathbf{0 . 7 9}$ & 0.59 & 0.46 & 0.35 & $\mathbf{0 . 8 3}$ \\
BC + IPR & 0.33 & 0.38 & 0.58 & $\mathbf{0 . 7 7}$ & 0.55 & 0.56 & 0.43 & $\mathbf{0 . 9 0}$
\end{tabular}

Table 3.5: Root mean square errors calculated over all volumes for each approach and each parameter with the non-interleaved data set

\subsubsection{Synthetic data: non-DW images at the beginning of the acquisition}

Estimated movements versus theoretical movements

The second set of synthetic data was simulated with 7 non-DW images at the beginning of the acquisition, it is referred as the non-interleaved data set. In that case, none of the three tested methods (IPR, coregistration and coregistration combined with IPR) was giving highly accurate results. This is confirmed by the RMSE summarized in Table 3.5. The coregistration approaches (BC and $\mathrm{BC}+\mathrm{IPR}$ ) give similar results to the first case with interleaved non-DW images $\left(T_{X Y Z}=0.79 / 0.77\right.$ and $\left.R_{X Y Z}=0.83 / 0.90\right)$ compared to BC $\left(T_{X Y Z}=0.97 / 0.79\right.$ and $\left.R_{X Y Z}=1.19 / 0.87\right)$. The IPR approach fails to give accurate results with $T_{X Y Z}=1.16$ and $R_{X Y Z}=2.13$.
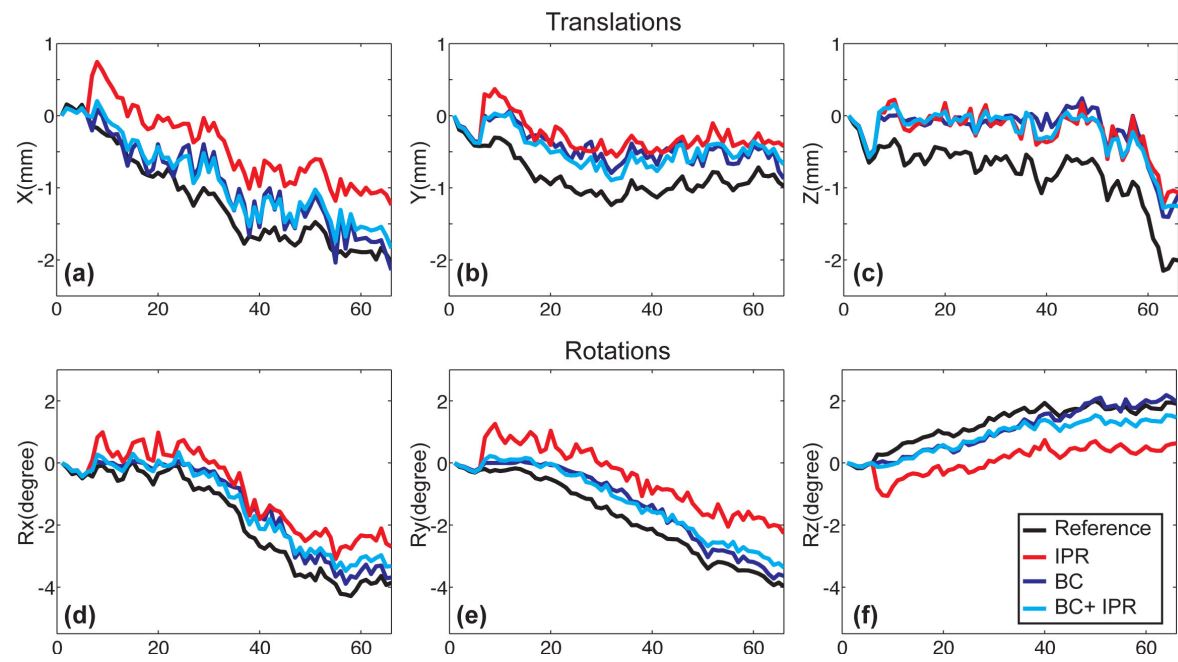

Figure 3.4: Estimated movements obtained the different approaches compared to the reference, in the case where all non-DW images were acquired before the $D W$ images. The $x$-axis represents the volume number and can be seen as a time representation. 


\section{Tensor distance maps}

The tensor distance maps confirmed that IPR is performing poorly as shown in Figure 3.5 and Table 3.6. The map is very similar to the one quantifying the distances without any realignment, as confirmed by a mean tensor distance of 0.22 compared to 0.23 . After $\mathrm{BC}$ or $\mathrm{BC}+\mathrm{IPR}$, the global distance is reduced and similar $(T D=0.15)$ : there is no difference between these two procedures. The two ROIs are delineated in green (ROI1 in the corpus callosum, ROI2 in the WM). For the first ROI in the corpus callosum, TD is only slightly reduced with BC (0.51 compared to 0.54 for IPR) which is not the case in the second ROI where the difference is high (0.04 compared to 0.22$)$.

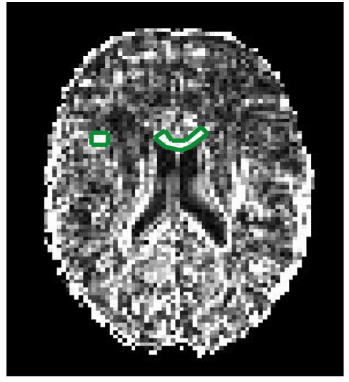

(a) Noisy VS Reference

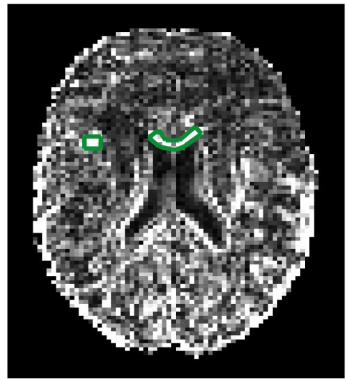

(c) IPR VS Reference

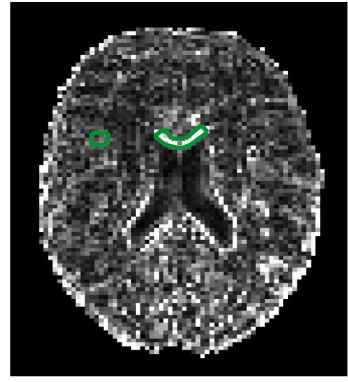

(b) BC VS Reference

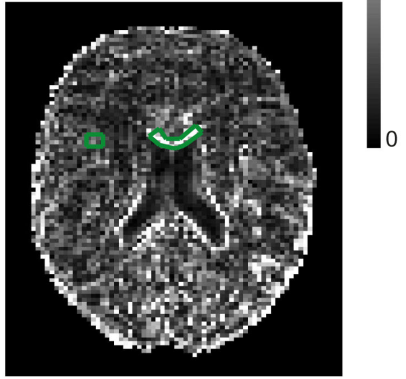

(d) BC + IPR VS Reference

Figure 3.5: Tensor distance maps for the case where all non-DW images are acquired before the DW images. ROI1 and ROI2 are indicated in green.

FA and main diffusion direction

As for TD, the errors in FA estimation as well as in the main diffusion direction are higher after correction with IPR, respectively 49.56 and $30.49^{\circ}$ for the whole brain volume. Both $\mathrm{BC}$ and $\mathrm{BC}+\mathrm{IPR}$ perform better with an error of 41.38 and 40.82 for FA and an error or $21.09^{\circ}$ and $21.19^{\circ}$ for the main direction. In the corpus callosum (ROI1), all the approaches perform 


\begin{tabular}{l|cccc} 
Approach & Whole volume & WM volume & ROI 1 & ROI 2 \\
\hline Noisy & 0.23 & 0.36 & 0.89 & 0.28 \\
IPR & 0.22 & 0.31 & 0.54 & 0.22 \\
BC & 0.15 & 0.24 & 0.51 & 0.04 \\
BC + IPR & 0.15 & 0.24 & 0.51 & 0.04
\end{tabular}

Table 3.6: Mean tensor distances after and before motion correction in four different volumes of interest - Case of non interleaved non-DW images

\begin{tabular}{l|cccc} 
Approach & Whole volume & WM volume & ROI 1 & ROI 2 \\
\hline Noisy & 57.95 & 51.04 & 1.10 & 0.80 \\
IPR & 49.56 & 44.15 & 0.77 & 0.75 \\
BC & 41.38 & 38.55 & 0.73 & 0.36 \\
BC + IPR & 40.82 & 37.94 & 0.72 & 0.36
\end{tabular}

Table 3.7: Error on FA after and before motion correction in four different volume of interest - Case of non interleaved non-DW images

the same in term of angle discrepancy $(\alpha=3.7)$. The FA error is almost the same with a value of 0.77 for IPR and 0.73 for BC. However in the WM area (ROI2), the errors are reduced by half for BC: 0.36 and $14.98^{\circ}$ compared to 0.75 and $34.18^{\circ}$.

\subsubsection{Stopping criterion}

An important step of IPR processing is the stopping criterion. Figure 3.6 shows the evolution of the global tensor distance after each iteration in the second case (non-interleaved data set): the new tensor maps are compared to the previous ones. The distance clearly decreases after few iterations to reach a plateau. Thus, we decided to stop it when the distance starts to increase. In that case, it is after 12 iterations: the corrected data will be then obtained after 11 iterations.

\begin{tabular}{l|cccc} 
Approach & Whole volume & WM volume & ROI 1 & ROI 2 \\
\hline Noisy & 33.49 & 26.16 & 5.14 & 50.35 \\
IPR & 30.14 & 22.74 & 3.70 & 34.18 \\
BC & 21.09 & 14.52 & 3.72 & 14.98 \\
BC + IPR & 21.19 & 14.69 & 3.67 & 14.13
\end{tabular}

Table 3.8: Error on the main fiber direction after and before motion correction in four different volumes of interest - Case of non interleaved non-DW images 


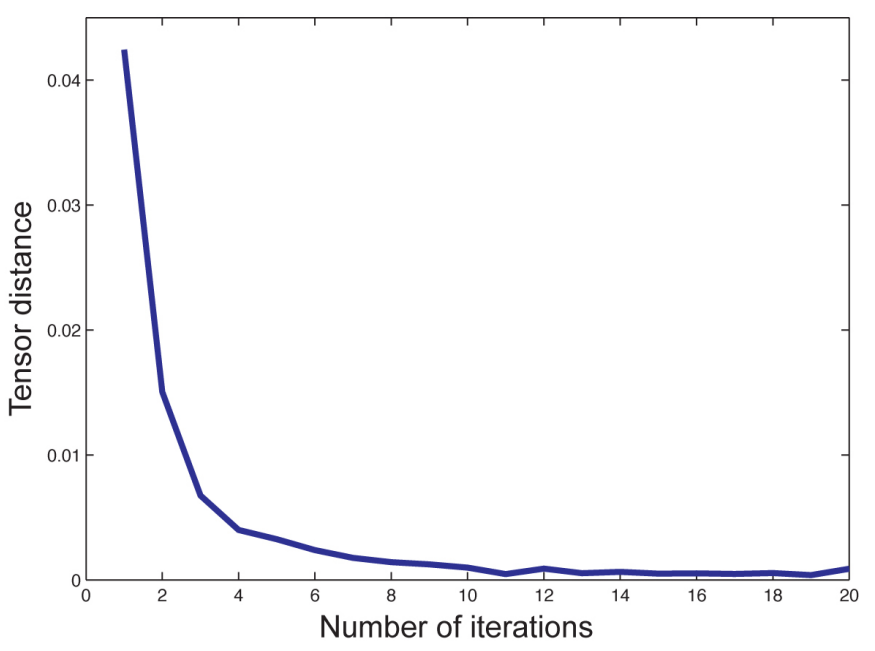

Figure 3.6: Stopping criterion evolution over 20 iterations.

\subsubsection{Real data}

\section{Comparison of estimated movements}

As those are real data, there is no reference in that case. However, the results shown in Figure 3.7 seem to confirm that interpolation and IPR are a good combination. If interpolation is assumed to do a good job for non-DW data, movements between two non-DW image should more or less follow the interpolated data (green line). BC procedure fails, in particular for the rotation around the $\mathrm{z}$-axis where the estimated rotations for DW images is shifted compared to the ones estimated for non-DW images.

\section{Effect on TD maps}

In order to visualize where are the differences in the tensor images after each correction, we compared the tensor distances, as shown in Figure 3.8 . The tensor distances between the tensors estimated after interpolation correction or after interpolation combined with IPR are very low, meaning the tensors and thus the derived parameters are very similar. When comparing the tensors estimated after coregistration correction with the two other methods, differences appear in the occipital area and on the boundaries with the CSF. 

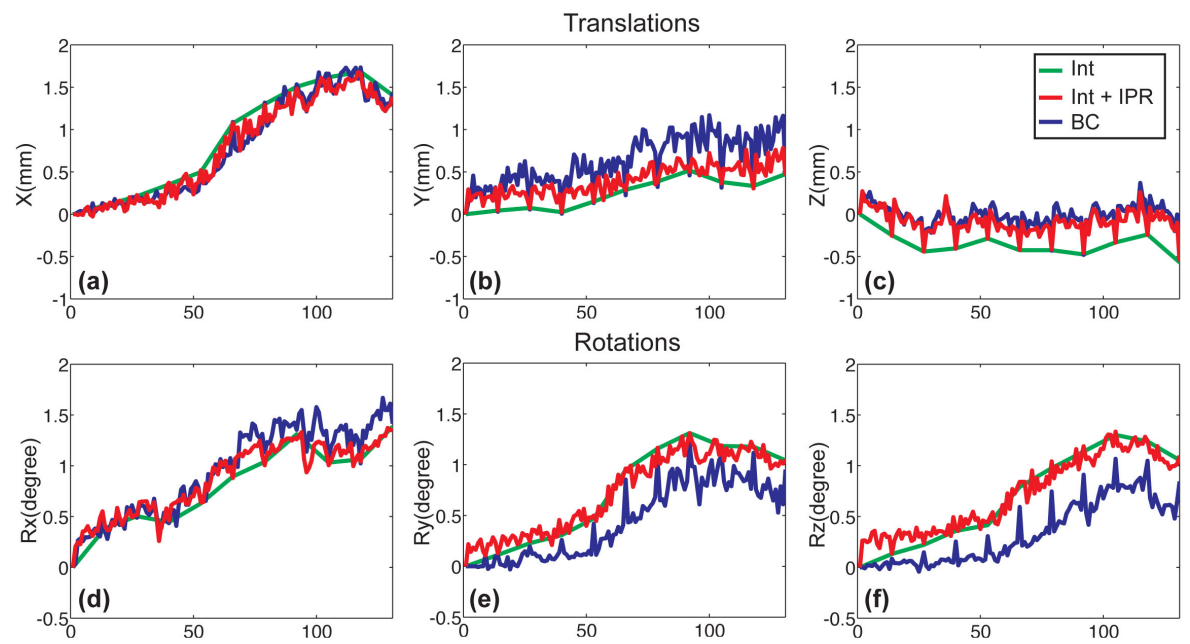

Figure 3.7: Estimated parameters for a real data set. The x-axis represents the volume number and can be seen as a time representation.

\subsection{Discussion}

In this chapter, a novel model-based method combined with a new tensor distance measure was investigated. This tensor distance can also be used as an error measure and using this distance we have quantified how misregistration creates bias in the tensor estimation. These errors propagate in term of traditional metrics such as FA or/and influence the estimated main direction of the fiber. It is thus important to correct for it.

In an ideal simulated case, this method outperforms other tested approaches, in particular the coregistration procedure, which is the most popular approach. We have also shown that a basic approach using coregistration of

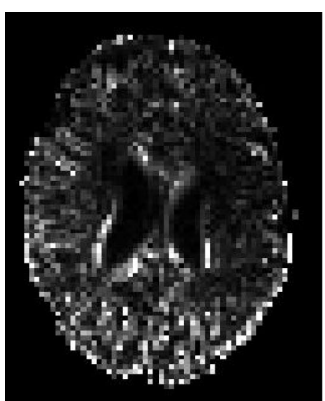

(a) BC VS Int

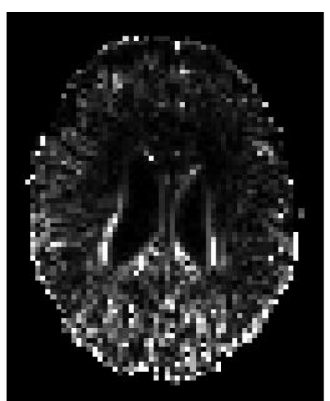

(b) BC VS Int+IPR

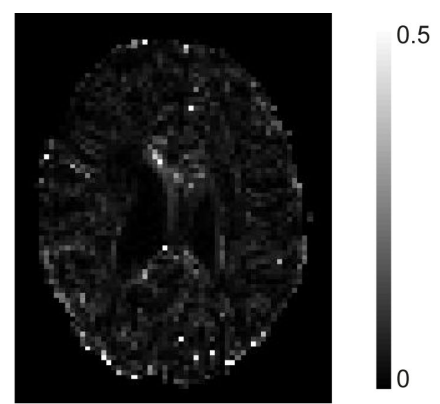

(c) Int VS Int+IPR

Figure 3.8: Tensor distance maps between the three methods 
interleaved non-DW images and interpolated movements is quite reliable and is a good approximation for real cases. The data set used as an example is very representative of the type of data acquired for clinical studies. The movement of the patient stays relatively smooth over the acquisition period, which confirms that our simulations are close to reality and that interpolation can be a good approximation.

The main limit of this work is that IPR performs well only when non-DW images were interleaved, which is not the case in most acquisitions, where non-DW images are all acquired at the beginning of the acquisition or only one image is acquired. However, as observed in Figure 3.4, DW images are better realigned between themselves than using coregistration (the red line is more parallel to the black on, as compared to the blue ones), despite a significant shift with the first nDW images. The scan-to-scan movements seem properly captured but there is a realignment error between the DW and nDW images. A possible explanation comes from the way theoretical images are generated using Eq. 3.11. The initial tensor from raw data is computed in the frame of the averaged position over all the volumes. The non-DW images being all at the beginning do not have the same averaged position, creating a shift between the two kinds of images. With the interleaved approach the averaged non-DW volumes are +/- in line with the DW volumes and tensor estimates are ok. To overcome this issue, an outlier detection step could be added in order to get a good tensor estimation after the first step, as suggested earlier by Bai (2009).

This study focused on DTI, where images are acquired in a low range of $b$-value, and the resulting SNR is quite high. In acquisition with higher $b$ values (up to $5000 \mathrm{~s} / \mathrm{mm}^{2}$ ), SNR is lower and due to long diffusion time, the signal drops into the noise level in most of the diffusion directions. The edges between WM and CSF, or between the brain and the air around are totally blurred, which will make coregistration even more difficult. One study by Ben-Amitay et al. (2012) using a similar model-based method, using a nongaussian model confirms this effect. They demonstrated a high impact of motion correction method on the resulting parameter maps, which confirms our hypothesis. Morevore, in order to improve our method, correction of distortions such as eddy-current distortions should be added.

Another realignment issue for group analysis is the inter-subject realignement. We did not treat this problem here but one can imagine using the tensor distance metric used here as a stopping criterion to coregister or to evaluate the mean of a group, instead of coregistering FA maps to a template which is currently the most popular method.

To conclude, we have demonstrated that our procedure is a reliable and efficient way to motion correct DW images, and that, with a proper ac- 
quisition setup, it performs better than standard coregistration procedures. The method must be improved to take into account any kind of acquisition schemes and should be extended to non-gaussian models as well. 


\section{Chapter 4}

\section{Noise correction for DKI experiments}

This chapter is based on: E.D. André, F. Grinberg, E. Farrher, I.I. Maximov, N.J. Shah, C. Meyer, M. Jaspar, V. Muto, C. Phillips and E. Balteau. Influence of Noise Correction on Intra- and Inter-subject Variability of Quantitative Metrics in Diffusion Kurtosis Imaging, PlosOne 9(4): e94531, 2014

\subsection{Introduction}

MRI is, intrinsically, a low SNR technique and the acquired signal is often heavily influenced by noise. The main source of noise is the thermal noise, also called Johnson noise. It originates from the thermal agitation of the charges in the coil and in the sample, the latter being the strongest source (Hoult and Lauterbur, 1979). Amplifiers and electronics are a source of numerical noise as well, however these sources are usually negligible. The noise depends also on acquisition parameters: image resolution, field of view, number of averaging, etc. The noise variance as a function of these parameters has been discussed by Parker and Gullberg (1990), Macovski (1996). In particular, Macovski (1996) emphasized that SNR is proportional to the voxel volume and the squared root of the acquisition time and is independent of the bandwidth of the received signal.

Knowledge about noise variance is useful for a number of applications. First, it is a good measure of image quality and reflects also the system performance. The estimation of the noise standard deviation is used to characterize image quality and to measure SNR (McGibney and Smith, 1993, Dietrich et al., 2007). Secondly, it is very often used as an input in image processing. In practice, accurate knowledge of the noise standard deviation can be required in several cases: segmentation (Brummer et al., 1993), filtering methods for denoising (Maggioni et al., 2013, Raya et al., 2008, 
Manjon et al., 2010, Golshan et al., 2013, Lam et al., 2013), estimation of diffusion tensor (Tristan-Vega et al., 2012, Veraart et al., 2011). In diffusion MRI, noise can be a real issue, especially for DKI due to the need of DW signal at higher diffusion weighting coefficients ( $b$-values up to 2500 $3000 \mathrm{~s} / \mathrm{mm}^{2}$ ). For conventional DTI ( $b$-values up to $1000 \mathrm{~s} / \mathrm{mm}^{2}$ ), SNR is still relatively high and the impact of noise on the estimation of parameters such as FA or MD is relatively small (Jones and Basser, 2004). However, as diffusion weightings become larger the signal drops rapidly, so is the SNR and the signal can easily reach the noise floor (Koay et al. 2009). This is particularly true in regions experiencing fast signal decay because of the free diffusion of the molecules (CSF) or because of the high degree of directionality along a specific direction (e.g. along the fibers in the WM). When the signal is about or below the noise floor, the noise introduces a significant bias artificially enhancing the measured signal intensity (Kristoffersen 2012). The noise is then interpreted as true signal and, if not corrected, leads to an overestimation of kurtosis. Thus in clinical applications, where the number of repetitions is limited by acquisition time, the low SNR and resulting noise bias can strongly affect the reliability and sensitivity of the diffusion experiment. For diagnosis purposes, as well as to derive medical inferences on the pathological alterations of the brain tissue, the accuracy and reproducibility of the estimated diffusion metrics are essential, which require to account or correct for noise bias.

In this work, we investigated the influence of noise correction on the estimation of DTI and DKI metrics, such as MD, FA or MK, in human data in vivo and their dependence on SNR. Two noise correction methods have been applied and compared with corrected and non-corrected data. Noise level was estimated prior model fitting, in order to fit the data to an estimated noise-free signal, and improve accuracy and precision of the diffusion model estimators. Two experiments were carried on:

Experiment 1 Intra-subject reproducibility of the DTI and DKI parameters estimation as a function of SNR was investigated. SNR was manipulated by repositioning the head of the subject within the multichannel head coil, taking advantage of the spatially varying sensitivity of each coil in the array, and by varying the spatial resolution.

Experiment 2 Inter-subject variability of DTI and DKI parameters was investigated in a group of 25 healthy volunteers to provide insight on the suitability and reliability of DTI and DKI metrics for group comparison in clinical studies. 
Hypothesis: noise correction reduces spurious intra- and inter-subject variability of the estimated parameters, providing more accurate and reproducible biomarkers.

\subsection{Signal distribution in magnitude MR images}

\subsubsection{Single channel coil: Rician distribution}

For a quadrature detector, it is generally assumed that the noise in each channel is a white Gaussian noise and is independent with zero-mean (Henkelman, 1985), meaning there is no noise correlations between the coils. After complex Fourier transform, Gaussian characteristics are preserved and the resulting complex signal is still Gaussian. However, real images are often replaced by magnitude images, to avoid phase shifts artifacts caused by eddy currents amongst others. In that case the signal $M$ follow a Rician distribution (Henkelman, 1985, Bernstein et al., 1989, Gudbjartsson and Patz, 1995):

$$
p(M)=\frac{M}{\sigma^{2}} \exp \left(-\frac{M^{2}+A^{2}}{2 \sigma^{2}}\right) I_{0}\left(\frac{A M}{\sigma^{2}}\right)
$$

where $\sigma^{2}$ is the variance of the Gaussian noise in the real and imaginary images, $A$ the noise-free signal and $I_{0}$ in the modified zero ${ }^{\text {th }}$ order Bessel function of the first kind. In the background, where signal is assumed to be null, this distribution transforms to a Rayleigh distribution:

$$
p(M)=\frac{M}{\sigma^{2}} \exp \left(-\frac{M^{2}}{2 \sigma^{2}}\right)
$$

At high SNR, the probability distribution of signal $M$ is well approximated by a Gaussian distribution with variance $\sigma^{2}$ and mean $\sqrt{A^{2}+\sigma^{2}}$ (Gudbjartsson and Patz, 1995):

$$
p(M)=\frac{1}{\sqrt{2 \pi \sigma^{2}}} \exp \left(-\frac{\left(M-\sqrt{M^{2}+A^{2}}\right)^{2}}{2 \sigma^{2}}\right)
$$

This is illustrated in Figure 4.13.

\subsubsection{Multichannels coil: Noncentral $\chi$ distribution}

In multiple coils experiment, the signal $S$ after inverse Fourier transform is described by (Aja-Fernandez and Tristan-Vega, 2012):

$$
S_{l}=A_{l}+\mathcal{N}_{l}\left(0, \sigma_{n}^{2}\right) \quad l=1, \ldots, L
$$



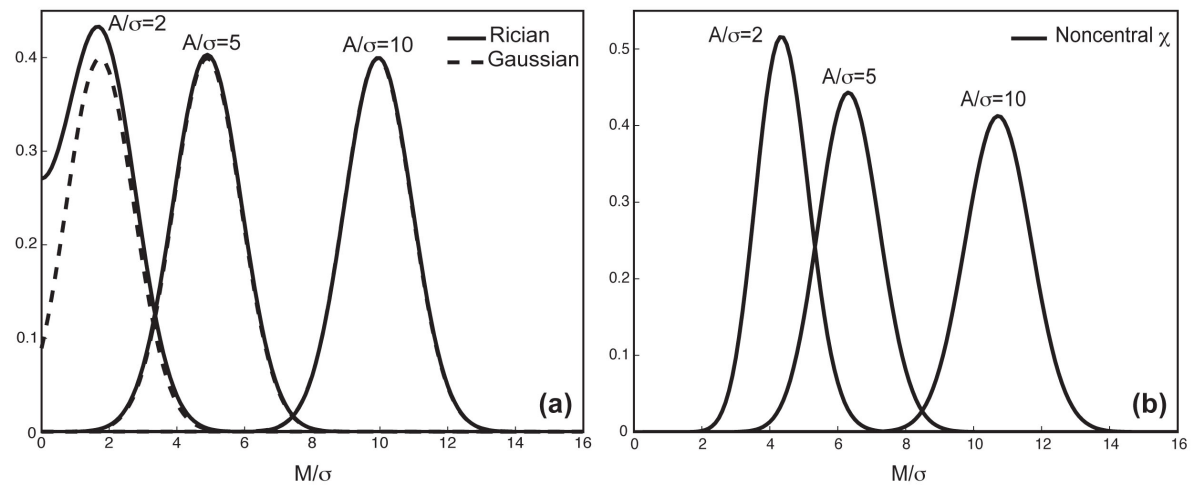

Figure 4.1: Probability density function of the signal for different SNR and different acquisitions, (a) Rician distribution can get approximated by a Gaussian distribution with mean $\sqrt{A^{2}+\sigma^{2}}$ and standard deviation $\sigma$ for higher SNR, (b) Noncentral- $\chi$ distributions for sum-of-square reconstruction with multichannels coil $(L=8)$

where $A_{l}$ is the noise-free signal, $\mathcal{N}_{l}$ is the complex white gaussian noise, with zero-mean and variance $\sigma_{n}^{2}$ and $L$ the total number of coils. After sum-of-square reconstruction, the signal $M_{L}$ is written as:

$$
M_{L}=\sum_{j=1}^{L}\left|S_{l}^{2}\right|
$$

This signal can be modeled by a noncentral $\chi$ (nc- $\chi$ ) distribution (Constantinides et al., 1997):

$$
p\left(M_{L}\right)=\frac{\eta_{L}}{\sigma^{2}}\left(\frac{M_{L}}{\eta_{L}}\right)^{L} \exp \left(-\frac{M_{L}^{2}+\eta_{L}^{2}}{2 \sigma^{2}}\right) I_{L-1}\left(\frac{M_{L} \eta_{L}}{\sigma^{2}}\right)
$$

where $L$ is the number of coils, $M_{L}$ is the measured signal, $\eta_{L}$ is the signal in the absence of noise (the "true" signal), $\sigma$ is the standard deviation of the noise and $I_{L-1}$ is the modified $(\mathrm{L}-1)^{\text {th }}$ order Bessel function of the first kind. The analytical expressions of the first and second moments of this distribution are given by Constantinides et al. (1997):

$$
\overline{M_{L}}=\sqrt{\frac{\pi}{2}} \frac{(2 L-1) ! !}{2^{L-1}(L-1) !}{ }_{1} F_{1}\left(-\frac{1}{2}, L,-\frac{\eta_{L}^{2}}{2 \sigma^{2}}\right) \sigma
$$

and

$$
\left\langle M_{L}^{2}\right\rangle=2 L \sigma^{2}+\eta_{L}^{2}
$$


respectively, where ${ }_{1} F_{1}$ is the confluent hypergeometric function and $(2 L-$ $1) ! !=1 * 3 * 5 * \ldots *(2 L-1)$. In the absence of signal $\left(\eta_{L}=0\right)$, the background noise follows a central $\chi$ distribution (Constantinides et al., 1997):

$$
p\left(M_{L}\right)=\frac{2^{1-L}}{\Gamma(L)} \frac{M_{L}^{2 L-1}}{\sigma^{2 L}} \exp \left(-\frac{M_{L}^{2}}{2 \sigma^{2}}\right)
$$

This distribution is a good approximation for sum-of-square (SoS) combination of the complex images from each of the coils (Constantinides et al. 1997), which is the reconstruction method used in this thesis. However, this approximation is valid only if the correlations between coils can be neglected (Aja-Fernandez and Tristan-Vega, 2012), which is not always the case, especially with modern multichannel coil with 32,64 or more channels. Parallel imaging techniques (Deshmane et al., 2012), in which $k$-space is partially sampled to speed the acquisition and reduce distortions, induce more complicated noise behaviour. For example, the noise can no longer be considered as spatially invariant, which makes background estimation invalid. This will be explained in more details in the following section.

\subsection{Noise correction methods}

\subsubsection{Noise estimation}

\section{Single acquisition methods}

The most straightforward method to estimate the noise is to use the background, where the signal is null. The delineation of the background has to be done with great care as it might be affected by ghosting artefacts. There are several ways to do so, either by manually delineating the region of the background or by using an automated procedure which get rids of artefacted voxels. The bigger the region, the more reliable the estimation is. Another way to get a background free from artefacts is to acquire a noise image, acquired with the radio-frequency $(\mathrm{RF})$ transmit amplitude set to zero for all RF pulses. A number of estimators have been proposed previously, which are reviewed in Aja-Fernandez et al. (2009). They are usually based on the first or second moments of the rician or nc- $\chi$ distribution. Another method based on histogram fitting can be used. However this method is not suitable for low SNR images, as the signal might fall into noise level and thus the noise peak of the histogram is mixed with the signal peak, resulting in biased estimation.

In this work, the estimation the noise standard deviation $\sigma$ was performed either from a noise image when available or from voxels extracted from the background of the DW images. In the latter case, a mask was created by 
automatically thresholding the mean non-DW image, excluding both signal and Nyquist ghost voxels. $\sigma$ was estimated prior to any processing using the following expression (Constantinides et al., 1997)

$$
\sigma=\sqrt{\frac{\sum_{i \in \text { background }} S_{i}^{2}}{2 L N}}
$$

where $S_{i}$ is the measured signal for each voxel in the background area, $N$ is the total number of background voxels and $L$ is the number of coils. The validity of Eq. 4.10 was confirmed in our experimental setup by inspecting the noise distribution in the acquired noise images. The expression given in Eq. 4.9 (with $\mathrm{L}=8$ ) closely fitted the histogram of measured noise voxel intensities, with a standard deviation closely matching the value calculated from Eq. 4.10 as shown in Figure 4.2(A) . The difference observed between the standard deviation estimated on the noise images and the standard deviation estimated on the background of the images in Experiment 1 did not exceed $1 \%$ when using DW images for background estimation and $6 \%$ when using non- DW images, as shown in Figure 4.2(B). This range of error has no significant impact on the noise correction procedure and the background noise estimation can therefore be used reliably when no noise image is available. In addition, the noise standard deviation is used to estimate the apparent local SNR in the images, calculated voxel-by-voxel as the mean signal of the non-DW images (prior noise correction) divided by the standard deviation of the noise.
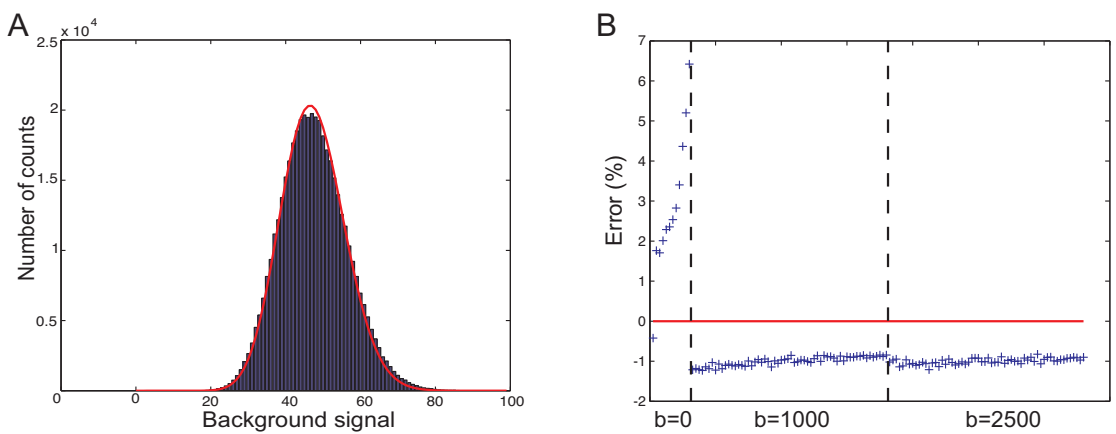

Figure 4.2: A. Histogram of a noise image with central $\chi$ distribution fit (solid red line). Fitting the histogram with the central $\chi$ distribution gives $\sigma=12,02$ and using the formula $\sigma=12.08$. B. Comparison of the noise estimation on the background of diffusion images compared to noise-only image using the background formula. Estimation on noise-only image is considered as the correct approximation. 


\section{Double acquisition methods}

Standard deviation can also be estimated using a double acquisition. These methods have been shown to be more robust (Sijbers et al., 1998) and can be used when no background is available: for example because it has been automatically suppressed by the manufacturer or because there is a restricted FOV (e.g. cardiac imaging). The difference between the two identical images is assumed to be a noise-only image (NEMA, 2001, Sijbers et al., 1998, Murphy et al., 1993) and the noise standard deviation can be estimated on that image. Artefacts such as ghosting or ringing do not influence this method, however, it is sensitive to misalignment between the two images and coregistration of the images will influence the resulting estimation due to smoothing and interpolation. This method also requires a double acquisition, which implies a double acquisition time: it is usually not clinically feasible.

Noise estimation in the case of parallel imaging reconstruction: spatial stationnarity

In the previous section 4.2.2, noise is assumed to be spatially invariant, that is, the standard deviation $\sigma$ is the same in each $k$-space acquisition point. This assumption is not always true and $\sigma$ might vary in space. It has been shown for parallel reconstruction (SENSE and GRAPPA) by Thunberg and Zetterberg (2007) with the help of numeric simulations. The particular case of SoS reconstruction has been investigated by Aja-Fernandez and Tristan-Vega (2012). They have shown that the signal distribution can be approximated by a nc- $\chi$ distribution where the real number of coil is replaced by an effective number of coil $L_{\text {eff }}$ and the standard deviation by an effective standard deviation $\sigma_{\text {eff }}$. This effective standard deviation is signal dependent: it depends thus on the location of the voxel. This means that the effect of correlations is the virtual reduction of the number of coil. This effect is more or less pronounced, depending on the number of coils (a large number of coils leads to stronger correlations) and if the $k$-space if fully sampled or not. In this work, the spatial variability of the noise standard deviation was assumed to be negligible and the background noise histogram fits the central- $\chi$ distribution pretty well (see Figure 4.2 (A)).

\subsubsection{Image denoising: non local mean filtering}

Image denoising, also called filtering has been a full area of research in MRI. Gaussian filtering is the most natural one but suffers from edge blurring. In order to preserve the information, other methods such as anisotropic 
filter (Gerig et al., 1992), total variation (Keeling, 2003), bilateral filtering (Wong et al., 2004), wavelet filtering (Nowak, 1999, Wood and Johnson 1999, Alexander et al., 2000) and non-local mean filtering (Raya et al., 2008. Coupe et al., 2008, Maggioni et al., 2013) have been developed. In general, these algorithms assume a Gaussian noise distribution and thus are not optimal for modern acquisitions, as described in the previous sections or must be applied to the complex raw data, which is not easily implemented.

In this work, a method similar to the one presented by Raya et al. (2008), will combined a denoising step and a noise bias correction, to get an unbiased denoising. In other words, the denoising step consists in getting a better estimation of the measured signal $S$, while correcting for the noise bias means estimating the true signal in absence of noise $\eta$. Prior to noise correction, a nonlocal mean filter (BM4D) (Maggioni et al., 2012) was optionally applied to the data. This filter provides a better estimation of the first and second moments of the measured magnitude, while preserving fine structures and details of the images. Its effect on the estimation of diffusion and kurtosis parameters was investigated at the individual and group level.

\subsubsection{First method for noise bias correction: the first moment correction}

This method, M1, is based on the first moment $\bar{M}_{L}$ of the noncentral chi distributed signal (Eq. 4.6). After realignment of the DW images (see Section 2.4), a look-up table of $\bar{M}_{L}$ versus $\eta_{L}$ was built for each individual image, using the estimated standard deviation and values of between the noise floor and the maximum measured intensity in the image. The noise floor is the minimum value taken by $\bar{M}_{L}$, corresponding to $\eta_{L}=0$. For example with $N=8$, the noise floor is equal to $3.94 \sigma$. For each pixel, the magnitude of the measured signal (after non local mean filtering if applied) was used as an estimate of the first moment $\tilde{M}_{L}$ while the true signal estimate $\tilde{\eta}_{L}$ was calculated by interpolation of the look-up table. The corrected signal intensity $\tilde{\eta}_{L}$ was set to zero whenever the measured intensity $\tilde{M}_{L}$ was below the noise floor.

\subsubsection{Second method for noise bias correction: Power image correction}

The second method, M2, is based on the expression of the second moment

(Eq 4.8). This method was first introduced by Miller and Joseph (1993) for single channel acquisitions and Gaussian signal distribution. The same approach is used here in the case of multichannel receiver coil and nc- $\chi$ distribution. The second moment of the signal distribution $E\left(M_{L}^{2}\right)$ is approximated by the square of the measured image $\tilde{M}_{L}^{2}$ (power image) after non-local mean filtering (if applied). Then, the correction is applied to the 
power image:

$$
\tilde{\eta}_{L}^{2}=\tilde{M}_{L}^{2}-2 L \tilde{\sigma}^{2}
$$

where $\tilde{\eta}$ is the true signal amplitude estimate, $\tilde{M}_{L}$ is the estimate of the first moment of the measured signal, $L$ is the number of coils, and $\sigma$ is the estimate of the noise standard deviation. When the measured signal intensity is below the noise floor, the squared true signal amplitude estimate is negative, leading to imaginary numbers in the corrected magnitude image. In such cases the corrected signal intensity $\tilde{\eta}_{L}$ was set to zero.

\subsection{Experimental protocol}

\subsubsection{Intra-subject inter-session variability}

The first experiment investigates the intra-subject variability of the DKI parameters as a function of SNR and relies on the assumption that for a single subject, the estimation of the DKI parameters should be reproducible over multiple sessions and SNR independent. The SNR was manipulated either by repositioning the head of the subject within the head coil, over several repetitions of the same measurement, taking advantage of the spatially varying sensitivity of each coil in the array (Protocol 1a), or varying the spatial resolution (Protocol 1b). Acquisition parameters are summarize in Table 4.1 .

\begin{tabular}{l|c|c|c} 
Parameters & Protocol 1a & Protocol 1b & Protocol 2 \\
\hline Number of repetitions & 1 & 1 & 3 \\
$b$-value $\left(\mathrm{s} / \mathrm{mm}^{2}\right)$ & $0 / 1000 / 2500$ & $0 / 1000 / 2500$ & $0 / 1000 / 2800$ \\
TR $(\mathrm{ms})$ & 7400 & 6800 & 7400 \\
TE $(\mathrm{ms})$ & 91 & 88 & 89 \\
FoV $(\mathrm{mm})$ & 211 & 192 & 192 \\
Number of slices & 54 & 44 & 58 \\
Matrix size & $88 \times 88$ & $64 \times 64$ & $96 \times 96$ \\
Voxel size $\left(\mathrm{mm}^{3}\right)$ & $2.4 \times 2.4 \times 2.4$ & $3 \times 3 \times 3$ & $2.2 \times 2.2 \times 2.2$
\end{tabular}

Table 4.1: Summary of the parameters of each protocols

Protocol 1a was repeated five times on the same volunteer (27 years old female) for different head positions (see Figure 4.3). Due to the spatially varying sensitivity of the coil array and the different positions of the head relatively to each coil element, the SNR was spatially dependent, and its spatial distribution varied from one session to the next. As a result, the SNR 
in a given brain area varied from one session to the next. In the following, the experiments related to Protocol 1a for different head positions will be referred to as P1 (center of the coil), P2 (shift up), P3 (shift down), P4 (shift to the right) and P5 (shift to the left). In Protocol 1b, data were acquired on the same volunteer with centered head position and larger voxel size in order to reach a global 2-fold increase in SNR. This high SNR protocol is expected to show moderate noise correction effects as compared to lower SNR situations. In the following, this protocol will be referred to as P6.

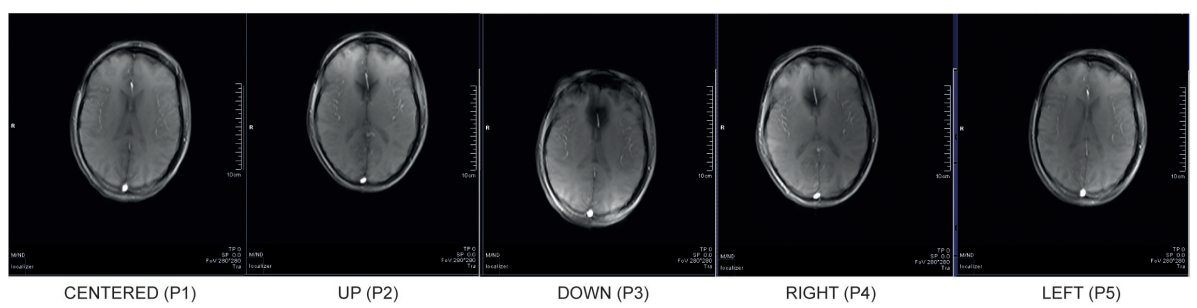

Figure 4.3: The different head positions for Protocol 1a. Images are displayed in radiological reference (left is right, right is left)

\subsubsection{Inter-subject variability}

Protocol 2 was acquired on 25 healthy male volunteers. Details are given in Table 4.1. In order to reduce the genuine inter-subject variability in the population, data sets were selected from a homogeneous population consisting of male volunteers recruited for an ongoing study with the following criteria: age range, 18-30 years old (mean $23 \pm 3$ ); non smokers with no history of neurological or psychological diseases and no medication or drug abuse. The amplitude and spatial distribution of the inter-subject variability was studied as a function of the noise correction procedure and SNR spatial distribution.

\subsubsection{Ethics statement}

The experimental procedures received approval from the Ethics Committee of the University of Liege and signed informed consent was obtained from all participants prior the beginning of the experiment.

\subsection{Global data processing}

\subsubsection{Data processing}

In all protocols, data were processed with the following steps: 
Motion correction According to the previous chapter about motion correction, images were realigned using linear interpolation. The IPR procedure could not be applied, as the DTI model is not applicable with high $b$-values. Thus non-DW images were first realigned with rigid body transformation using SPM8 (Wellcome Trust Centre for Neuroimaging, UCL, UK), for each individual session and movements between two non-DW images were interpolated. Transformations were applied to the corresponding DW images. Moreover, the diffusion directions were rotated accordingly (Leemans and Jones, 2009).

Noise correction The non-local mean filter was optionally applied and images were optionally corrected for noise with the two correction schemes described above. In total, six different procedures are compared:

- no correction (NC)

- non-local mean filtering only $(\mathrm{BM} 4 \mathrm{D}+\mathrm{NC})$

- first moment method (M1)

- non-local mean filtering and M1 (BM4D+M1)

- second moment method (M2)

- non-local mean filtering and M2 (BM4D+M2)

Kurtosis estimation As described in the introduction (section 2.4.3), the normalized signal intensities were fitted with the kurtosis model (Eq. 2.24 for each diffusion direction, on a voxel-by-voxel basis, using a nonlinear least square algorithm. Then, the diffusion tensors are estimated by solving a linear system for the tensor components (Basser et al., 1994) and diagonalized. In a similar fashion, kurtosis tensors were estimated voxel-by-voxel (Jensen et al., 2005). The kurtosis tensors were transformed from the laboratory coordinate system to a coordinate system defined by the three eigenvectors of the diffusion tensor (Hui et al., 2008). Conventional DTI (FA and MD) as well as DKI metrics (Jensen and Helpern, 2010) were evaluated. Among three kurtosis metrics (axial, radial, and mean), we focused our presentation on MK as the most representative and frequently used one. MK was calculated as the averaged apparent kurtosis (evaluated from the kurtosis tensor) over the unit sphere, as described by equation (55) in (Jensen and Helpern, 2010). 


\subsubsection{Assessment of intra-subject variability}

\section{Normalization}

In order to compare the different sessions, non-DW images were realigned in the image space of the first session and the same spatial transformations were applied to the parameter maps. Few outlier voxels with extremely high fitted values of MK were reassigned with the averaged neighboring values.

\section{Region of interest analysis}

Region of interest (ROI) analysis was performed on eight independent regions. These regions were delineated in six different WM and two GM areas using the Harvard-Oxford subcortical structural atlas and the JHU white-matter tractography atlas available in FSL (Analysis Group, FMRIB, Oxford, UK (Jenkinson et al., 2012)), Temporal Lobe (TL), Internal Capsule (IC), Anterior Corona Radiata (ACR), Globus Pallidus (GP),both left and right. These ROIs are illustrated in Figure 4.4. MK values from these ROIs were extracted and compared. Pearson's linear correlation tests were performed to determine if the mean MK was significantly correlated or not to SNR for each ROI and each correction separately.

\section{SNR maps}

SNR maps were derived for each session as the ratio of the mean non-DWI signal and the standard deviation of the noise.

\section{Z-scores map}

Z-scores (or standard scores) are a widely used method to the deviation of each individual measure from the average, in unit of standard deviation. The $\mathrm{z}$-score of a individual score $\mathrm{x}$ is:

$$
z=\frac{x-\mu}{\sigma}
$$

where $\mu$ is the mean of the sample and $\sigma$ is the standard deviation of the sample. This score was calculated voxel-by-voxel for MK maps for each of the corrections.

\subsubsection{Assessment of inter-subject variability}

\section{Normalization}

For group analysis, data from the 25 subjects were normalized to MNI spaces. The mean non-DW images were individually segmented and warped 

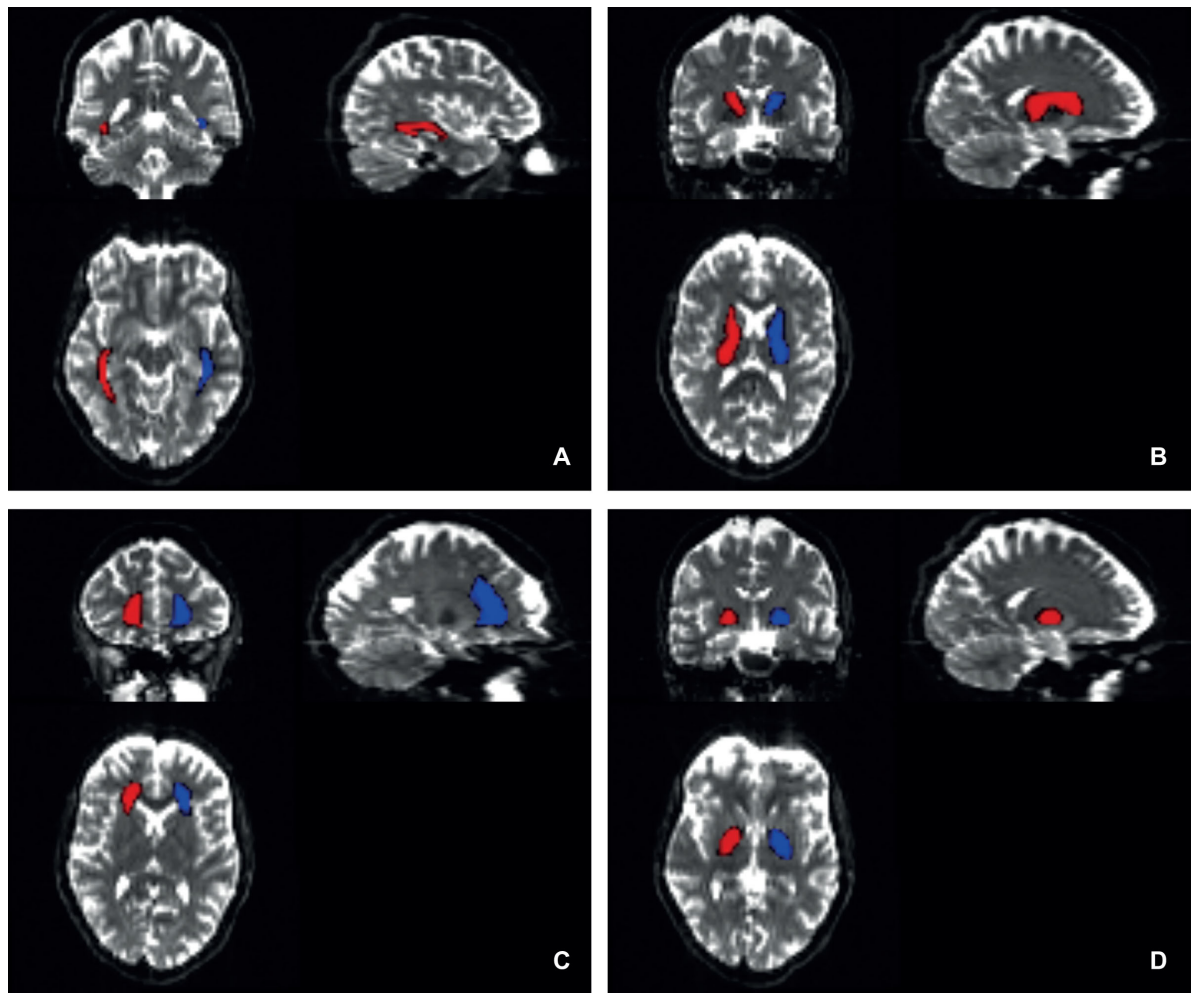

Figure 4.4: Localization of the four different ROIs on a non-DW image in the left in red and in the right in blue. A. Temporal lobe. B. Internal capsule. C. Anterior corona radiata. D. Globus pallidus.

into MNI space using the new segment tool in SPM8 (Wellcome Trust Centre for Neuroimaging, UCL, UK). The same non-linear transformations were applied to the scalar parameter maps.

Global analysis

For MD, FA and MK maps respectively, standard deviation maps across the group were calculated for each noise correction scheme and histograms of the MD, FA and MK maps averaged over the 25 subjects were also calculated. GM and WM histograms of MK maps were calculated using corresponding masks. These masks were created using FA maps thresholded at $<0.25$ for GM and $>0.25$ for WM. 


\section{Region of interest analysis}

ROI analysis using the ROIs described above (Experiment 1) was also performed on each of the 25 subjects, and the ROI mean and standard deviation of MK over the 25 subjects were calculated for each noise correction scheme and compared.

\subsection{Results}

\subsubsection{Effect of noise correction}

Figure 4.5 shows the effect of the different correction schemes on the signal decay for two voxels with significantly different SNR (26.3 and 16.7) in WM. The fitted values of $D_{a p p}$ and $K_{a p p}$ (Eq. 2.24) for the different correction schemes are reported in Table 4.2. Correction has a stronger effect for higher $b$-values and/or for low SNR data points. As a result, the estimation of the apparent kurtosis Kapp appears strongly affected by the noise bias: the difference between non corrected and corrected values reaches about 25-30\%. In contrast, the estimation of $D_{a p p}$ is less strongly affected in all correction schemes (up to 10\%). This is primarily due to significantly higher SNR and smaller noise bias observed for data points acquired at low diffusion weightings ( $b=0$ or $1000 \mathrm{~s} / \mathrm{mm}^{2}$ ), which predominantly determine the estimated $D_{a p p}$ value. The influence of BM4D filtering on $D_{a p p}$ and $K_{a p p}$ estimates appears negligible which is not surprising since the filtering procedure only reduces the local variance but does not correct for the noise bias.

\begin{tabular}{lcccc} 
& \multicolumn{2}{c}{$\mathrm{SNR}=26.3$} & \multicolumn{2}{c}{$\mathrm{SNR}=16.7$} \\
\hline & $K_{a p p}$ & $D_{a p p}$ & $K_{a p p}$ & $D_{a p p}$ \\
$\mathrm{NC}$ & $0.97 \pm 0.03$ & $0.84 \pm 0.02$ & $1.29 \pm 0.01$ & $0.93 \pm 0.01$ \\
$\mathrm{BM} 4 \mathrm{D}+\mathrm{NC}$ & $0.99 \pm 0.02$ & $0.85 \pm 0.01$ & $1.27 \pm 0.01$ & $0.84 \pm 0.01$ \\
$\mathrm{M} 1$ & $0.74 \pm 0.03$ & $0.85 \pm 0.01$ & $0.89 \pm 0.01$ & $1.00 \pm 0.01$ \\
$\mathrm{BM} 4 \mathrm{D}+\mathrm{M} 1$ & $0.76 \pm 0.02$ & $0.87 \pm 0.01$ & $0.84 \pm 0.01$ & $0.90 \pm 0.01$ \\
M2 & $0.67 \pm 0.04$ & $0.83 \pm 0.01$ & $0.89 \pm 0.01$ & $1.00 \pm 0.01$ \\
BM4D+M2 & $0.75 \pm 0.01$ & $0.87 \pm 0.01$ & $0.79 \pm 0.02$ & $0.90 \pm 0.01$
\end{tabular}

Table 4.2: $K_{\text {app }}$ and $D_{\text {app }}\left(10^{-3} \mathrm{~mm}^{2} / \mathrm{s}\right)$ values for two voxels with different SNR, corresponding to Figure 4.5, with their standard errors.

A map of $M K$ obtained using the noise correction method M1 is shown in Figure 4.7(a), as an example. The corresponding histograms of $M K, M D$, and $F A$ are shown in Figures 4.7(b), 4.7(c), and 4.7(d), respectively. In the absence of correction, $M K$ values are strongly overestimated as demonstrated by the histograms in Figure 4.7(b). For both correction schemes, 

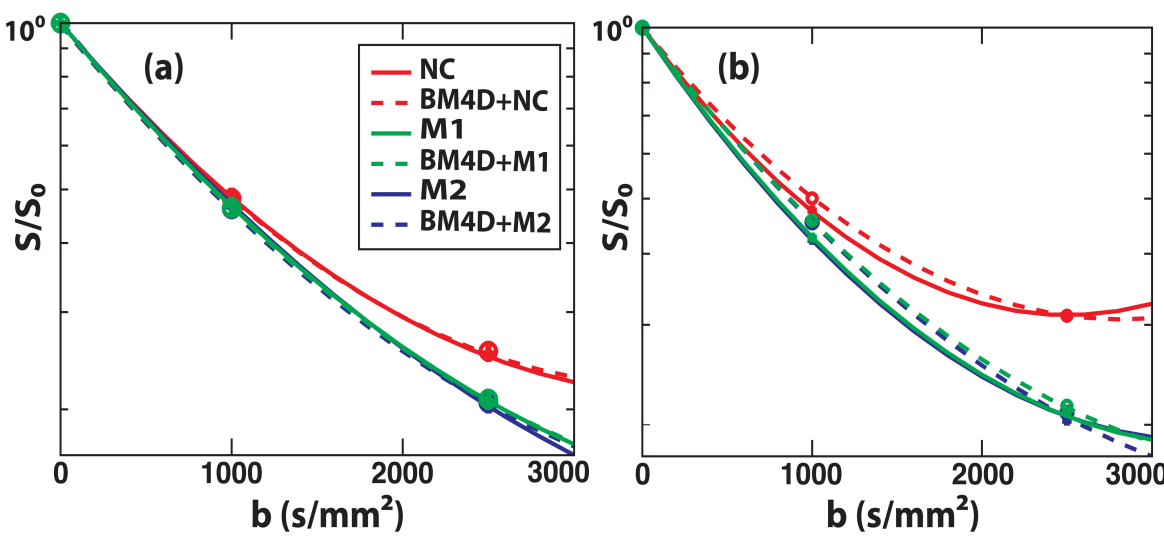

Figure 4.5: Normalized signal attenuation curves before and after noise corrections together with their DKI fits for the different correction schemes. The data correspond to a single diffusion direction in WM areas, for two different voxels with apparent SNR values equal to 26.3 (a) and 16.7 (b) respectively. Corresponding values of $K_{a p p}$ and $D_{a p p}$ are summarized in Table 4.2.

M1 and M2, the histograms exhibit significant shifts of both WM and GM peaks towards lower $M K$ values as compared to non-corrected data: from 1.4 to 1.05 for WM and from 0.75 to 0.6 for GM. The two correction methods show similar results whereas BM4D filtering produces no significant effect. Figure 4.8 compares Protocol 1a (P1) with low SNR acquisition and Protocol 1b (P6) with higher SNR acquisition for the same slice as in Figure 4.7. After correction (M1) $M K$ maps are similar for low and high SNR acquisition, as shown in Figures 4.7(a) and 4.8(a). The corresponding $M K$ histograms for three corrections schemes (NC, M1 and M2) are shown in Figure 4.8(b). Similar histograms were obtained with corrections schemes using BM4D filter (not shown). The histogram of non-corrected data at high SNR (dashed red line) exhibits a clear shift compared to non-corrected data at lower SNR (solid red line) towards lower $M K$ values. Moreover, the difference between non-corrected and corrected data becomes smaller at high SNR. In accordance with the analysis of the signal decay in Figure 4.5 the impact of the noise correction on the estimation of $M D$ and FA derived from the diffusion tensor is practically negligible, as demonstrated by their histograms in Figures 4.7(c) and 4.7(d). A slight shift towards higher mean diffusivity is observed in the MD histograms, since noise correction generally tends to increase the slope of the DW signal decay. The fractional anisotropy is almost unaffected, indicating a much smaller bias in estimated $F A$ maps when no noise correction is applied. 


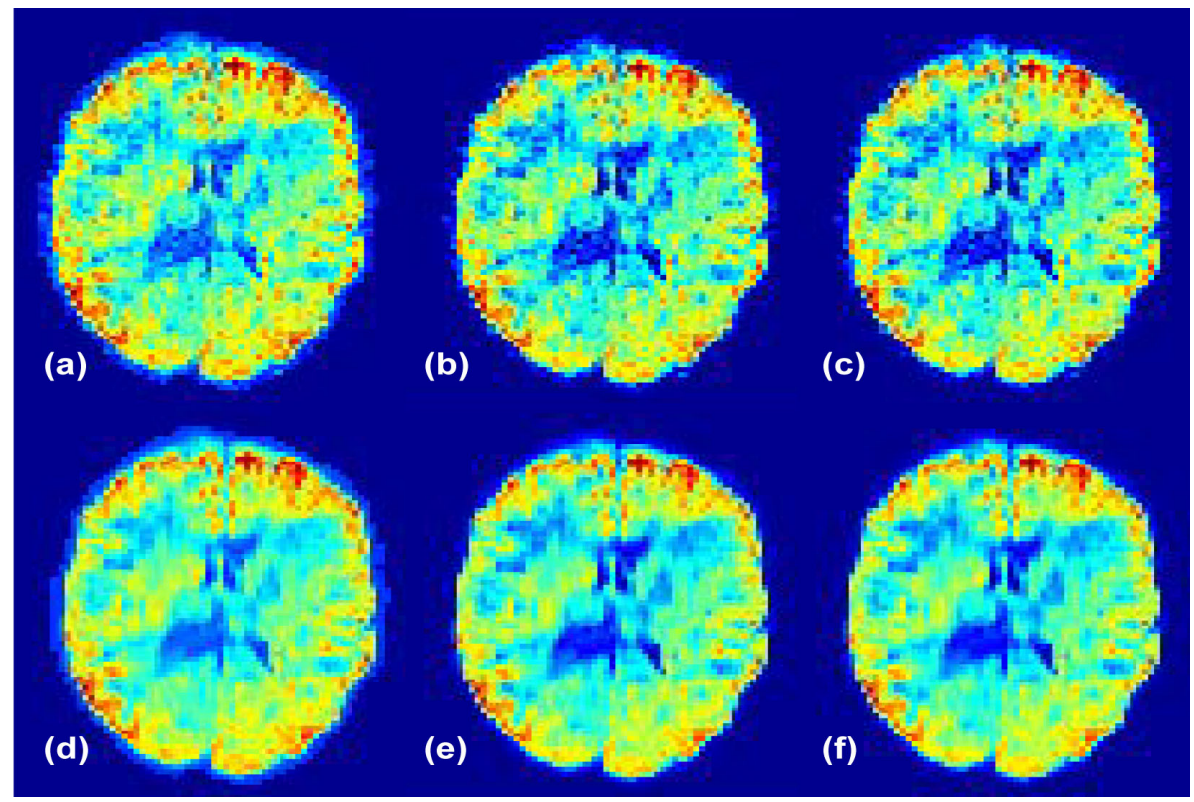

Figure 4.6: Visual inspection of the effect of noise corrections on a $D W$ image $\left(b=1000 \mathrm{~s} / \mathrm{mm}^{2}\right.$. (a) Raw image (NC), (b) M1, (c)M2, (d) $B M 4 D+N C$, (e) $B M 4 D+M 1$, (f) $B M 4 D+M 2$

\subsubsection{Intra-subject variability}

Results from the experiment 1 are shown in Figure. 4.9, 4.10 and 4.11, In this experiment, the same measurement protocol was repeated 5 times for various positions of the head of the subject in the coil.

\section{SNR dependence of $M K$}

The spatial distribution of SNR across the head was different in each of the measurements (P1 to P5), as illustrated in Figure 4.9. The lower spatial resolution in $\mathrm{P} 6$ leads to a global 2-fold increase of SNR. In Figure 4.9, we compared the $M K$ maps obtained after BM4D filtering and with and without noise correction. $M K$ maps obtained without BM4D filtering were similar and therefore not shown. In the absence of noise correction, the $M K$ estimate is systematically higher and depends significantly on the SNR. For example, the maps obtained at lower SNR (P1 to P5) exhibit higher values than the one obtained at higher SNR (P6). Besides, we observe that the regions with enhanced $M K$ values correlate with lower SNR regions depending on the head position. This effect is further illustrated in Figure 4.9(b), by a closer view of the region delineated in Figure 4.9(a), for experiments 
$\mathrm{P} 1, \mathrm{P} 4, \mathrm{P} 5$ and $\mathrm{P} 6$. In that region, the SNR (and the $M K$ estimates accordingly) varies strongly from one acquisition to the next when no correction is applied (Figure 4.9(b), second column). The regions of lower SNR exhibit higher $M K$ values. This effect is particularly marked in the region delineated by the black rectangle (Figure 4.9(b)). M1 and M2 corrections lead to very similar $M K$ maps.

\section{$\mathrm{ROI}$ analysis}

The influence of noise correction on the evaluated metrics was quantitatively assessed by statistical analysis in 8 ROIs. Examples are represented in Figure 4.10 for the right GP and left TL where the $M K$ values averaged over the indicated ROIs are compared for all correction schemes (see different bar groups) and positions (see bars within a given group). The averaged SNR values of the non-DW images corresponding to each session are indicated on the plots. In all sessions, non-corrected $M K$ values remain higher than corrected ones. One can observe also that higher $M K$ values correlate with low SNR values when no correction is applied. For example, in the left

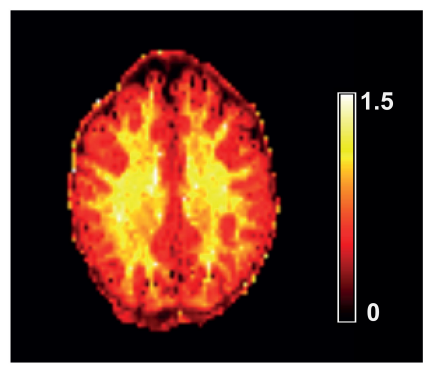

(a)
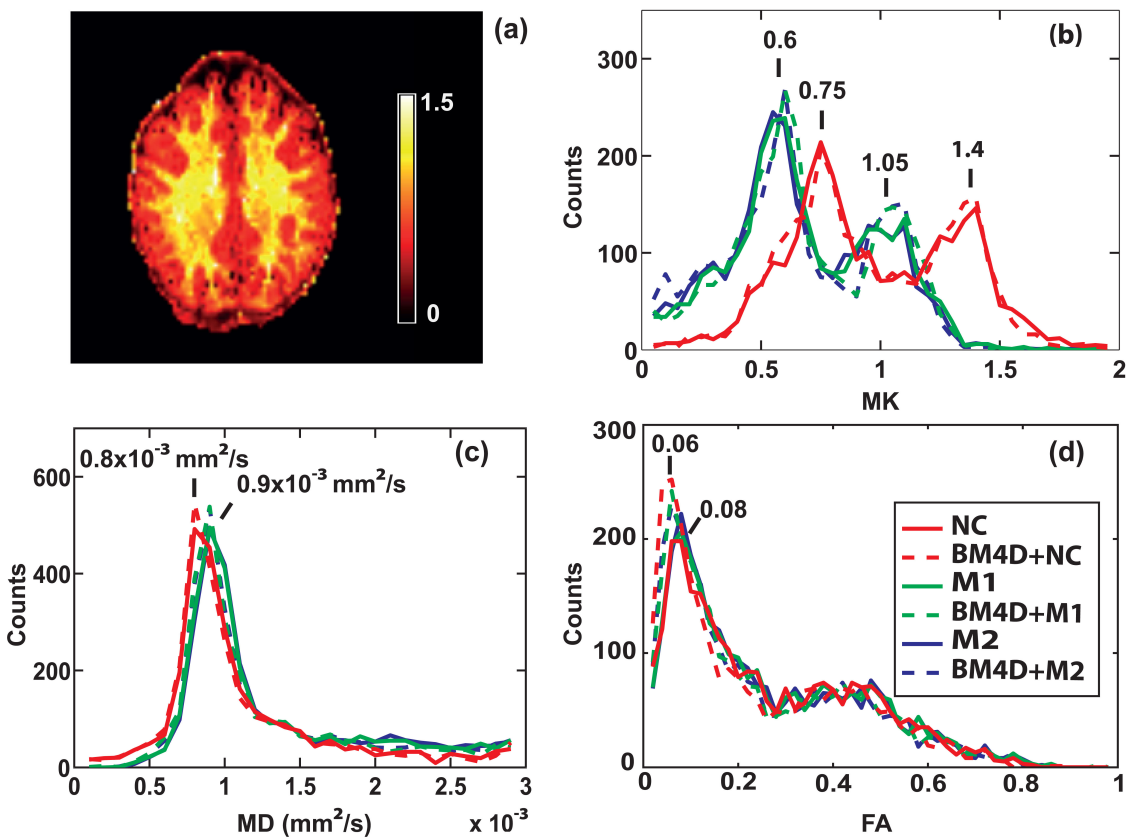

Figure 4.7: (a) MK map for one single subject (position P1) and one selected slice corrected with method M1. (b) MK, (c) MD and (d) FA histograms of the same slice for each noise correction scheme. 
(a)

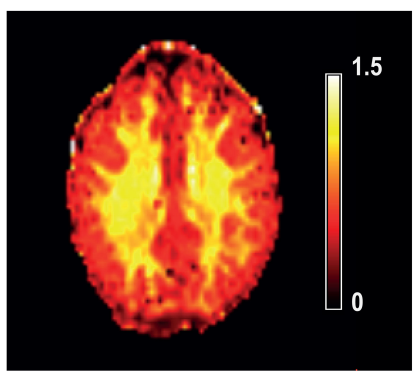

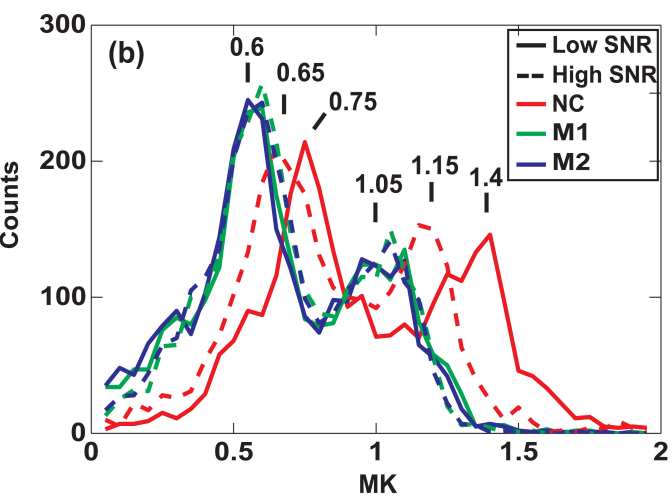

Figure 4.8: (a) MK map of the selected slice for high SNR acquisition (P6) corrected with $M 1$ and (b) the MK histograms of the same slice for three correction schemes (dashed lines). The corresponding histograms for P1 (low SNR acquisition) are also shown (solid curves). The histograms are practically overlapping after the noise correction (blue and green curves). The non-corrected histograms are shifted with respect to each other. However, the difference between corrected and non-corrected values is smaller for higher SNR.

TL (Figure 4.10(b)), lower SNR of 17.88 is associated with significantly higher $M K$ of 1.34 in comparison to the value of 1.19 evaluated at higher SNR of 23.30, that is a significant increase of $13 \%$. In contrast, when noise correction is applied, the MK estimate is globally lower and no longer dependent on SNR. For example, practically the same $M K$ values of 0.92 and 0.9 were obtained for SNR values of 17.8 and 23.3, respectively. Moreover, Figure 4.10 shows that the influence of noise is reduced at high SNR (e.g. $\mathrm{SNR}=34.53$ in $\mathrm{P} 6$ ) where the difference between $M K$ values estimated with and without noise correction exhibits an increase of about $16 \%$ while corresponding values obtained in sessions P1-P5 exhibits differences up to $50 \%$.

The results obtained for different ROIs are summarized in 4.3 , where the stars indicate the results of the Pearson's correlation tests. Generally, kurtosis values depend on the tissue (WM or GM) and can vary within an area consisting of a given tissue type. The mean $M K$ over the ROI for each position (P1 to P5) is significantly correlated to SNR for non-corrected data $(p<0.01)$ for all investigated ROIs. On the contrary, no correlations are reported for corrected data, except for two cases where a weak correlation is observed $(p<0.05)$. 


\begin{tabular}{lcccccc} 
& $\mathrm{NC}$ & $\mathrm{BM} 4 \mathrm{D}+\mathrm{NC}$ & $\mathrm{M} 1$ & $\mathrm{BM} 4 \mathrm{D}+\mathrm{M} 1$ & $\mathrm{M} 2$ & $\mathrm{BM} 4 \mathrm{D}+\mathrm{M} 2$ \\
\hline Right TL & $1.25 \pm 0.06^{* *}$ & $1.21 \pm 0.06^{* *}$ & $0.90 \pm 0.01$ & $0.90 \pm 0.01$ & $0.90 \pm 0.01$ & $0.87 \pm 0.01$ \\
Right GP & $1.36 \pm 0.06^{* *}$ & $1.37 \pm 0.06^{* *}$ & $0.92 \pm 0.02^{*}$ & $0.92 \pm 0.02$ & $0.88 \pm 0.01^{*}$ & $0.90 \pm 0.02$ \\
Right IC & $1.27 \pm 0.03^{*}$ & $1.25 \pm 0.04^{*}$ & $0.97 \pm 0.01$ & $0.93 \pm 0.01$ & $0.94 \pm 0.01$ & $0.91 \pm 0.01$ \\
Right ACR & $1.35 \pm 0.08^{* *}$ & $1.34 \pm 0.08^{* *}$ & $0.99 \pm 0.01$ & $0.99 \pm 0.01$ & $0.97 \pm 0.01$ & $0.96 \pm 0.01$ \\
Left TL & $1.18 \pm 0.08^{* *}$ & $1.15 \pm 0.07^{* *}$ & $0.89 \pm 0.02$ & $0.88 \pm 0.02$ & $0.87 \pm 0.02$ & $0.86 \pm 0.02$ \\
Left GP & $1.36 \pm 0.08^{* *}$ & $1.37 \pm 0.08^{* *}$ & $0.94 \pm 0.01$ & $0.97 \pm 0.01$ & $0.91 \pm 0.01$ & $0.95 \pm 0.01$ \\
Left IC & $1.27 \pm 0.05^{* *}$ & $1.25 \pm 0.05^{* *}$ & $0.97 \pm 0.01$ & $0.95 \pm 0.01$ & $0.96 \pm 0.02$ & $0.95 \pm 0.02$ \\
Left ACR & $1.31 \pm 0.06^{* *}$ & $1.29 \pm 0.06^{* *}$ & $0.97 \pm 0.01$ & $0.97 \pm 0.02$ & $0.95 \pm 0.02$ & $0.95 \pm 0.02$
\end{tabular}

Table 4.3: Mean MK values and standard deviations for each correction scheme and each ROI. Pvalues for Pearson correlation with SNR are indicated by: ${ }^{*} p<0.05$ and ${ }^{* *} p<0.01$. All correlations were negative.

\section{Z-score analysis}

The z-score maps shown in Figure 4.11 emphasize this effect at the voxel level. Z-score maps indicate the positive or negative deviation of individual $M K$ maps from the average MK map over protocols P1 to P5 for a given noise correction scheme, in units of the standard deviation. When no correction is applied, the z-score maps exhibit strong spatial heterogeneity (white arrows) in contrast to the homogeneous appearance of the z-score maps after correction.

\subsubsection{Inter-subject variability}

In this section we examine the influence of noise correction on inter-subject variability and on the contrast between WM and GM in $M K$ maps.

\section{Mean and standard deviation maps}

As an example, Figure 4.12 shows $M K$ maps of one selected slice (at the level of Corpus Callosum) averaged over 25 subjects (first and third rows) and the corresponding standard deviations across all subjects (second and fourth rows). As shown in the previous section on a single subject, the $M K$ is globally lower and the contrast-to-noise ratio (CNR) between WM and GM is higher with noise bias corrected data (Figure 4.12(b), 4.12(c), 4.12(e) and 4.12(f)) as compared to non-corrected data (Figure 4.12(a) and 4.12 (d)). Quantitatively, these results are illustrated by the histograms in Figure 4.13. After noise correction, the peaks of MK distribution are at 0.5 and 1.0, for GM and WM respectively, whereas, without noise correction, they are at 0.9 and 1.4 for GM and WM respectively. After correction, the peaks are significantly sharper and better separated, providing a better 
distinction between GM and WM. The effect of the BM4D filter is very subtle, leading to a slight increase of $M K(+0.05)$.

Inter-subject variability is investigated through the standard deviation maps of MK across the group in Figure. 4.12(a) 4.12(f) (second rows) and the mean SNR map of the non-diffusion weighted image over the 25 subjects (Figure $4.12(\mathrm{~g}))$. On the one hand, standard deviation maps show that non-corrected maps are much more heterogeneous than corrected ones. The standard deviations reach a value of 0.4 in the frontal area that is twice as large as a value of 0.2 in the occipital area. On the other hand, on the mean SNR map, we observe a spatial gradient of SNR from top to the bottom, that is, SNR tends to increase towards the bottom of the image. This effect is related to hardware properties, more precisely to the spatially varying sensitivity of the coil array. When comparing both standard deviation maps and SNR maps, one can infer that the enhancement of standard deviation in the upper regions of the non-corrected maps correlates with observed SNR gradient from top to bottom. This result provides evidence that higher intersubject variability of non-corrected MK maps is, in part, due to hardwarerelated SNR heterogeneity, and not only a genuine inter-subject variability.

\section{ROI analysis}

These results are supported by the ROI analysis averaged over 25 subjects. Numerical values of the MK are summarized in Table 4.4. In all ROIs and in the absence of noise correction, the MK averaged over all subjects is globally higher (by as much as about 50\%) in comparison to the corrected value. The standard deviation of the MK is also systematically higher if no correction is applied.

\begin{tabular}{lcccccc} 
& $\mathrm{NC}$ & $\mathrm{BM} 4 \mathrm{D}+\mathrm{NC}$ & $\mathrm{M} 1$ & $\mathrm{BM} 4 \mathrm{D}+\mathrm{M} 1$ & $\mathrm{M} 2$ & $\mathrm{BM} 4 \mathrm{D}+\mathrm{M} 2$ \\
\hline Right TL & $1.36 \pm 0.07$ & $1.34 \pm 0.07$ & $0.84 \pm 0.05$ & $0.85 \pm 0.03$ & $0.83 \pm 0.05$ & $0.81 \pm 0.04$ \\
Right GP & $1.85 \pm 0.17$ & $1.87 \pm 0.18$ & $0.81 \pm 0.04$ & $0.92 \pm 0.05$ & $0.78 \pm 0.03$ & $0.85 \pm 0.05$ \\
Right IC & $1.49 \pm 0.07^{*}$ & $1.87 \pm 0.18$ & $0.96 \pm 0.04$ & $0.94 \pm 0.04$ & $0.94 \pm 0.03$ & $0.90 \pm 0.02$ \\
Right ACR & $1.49 \pm 0.07$ & $1.87 \pm 0.18$ & $0.96 \pm 0.04$ & $0.94 \pm 0.02$ & $0.94 \pm 0.03$ & $0.90 \pm 0.02$ \\
Left TL & $1.30 \pm 0.08$ & $1.28 \pm 0.07$ & $0.86 \pm 0.04$ & $0.88 \pm 0.06$ & $0.86 \pm 0.05$ & $0.84 \pm 0.03$ \\
Left GP & $1.76 \pm 0.15$ & $1.79 \pm 0.17$ & $0.81 \pm 0.07$ & $0.92 \pm 0.04$ & $0.79 \pm 0.05$ & $0.86 \pm 0.04$ \\
Left IC & $1.45 \pm 0.06$ & $1.44 \pm 0.05$ & $0.96 \pm 0.03$ & $0.97 \pm 0.02$ & $0.96 \pm 0.02$ & $0.93 \pm 0.02$ \\
Left ACR & $1.41 \pm 0.07$ & $1.41 \pm 0.07$ & $0.89 \pm 0.04$ & $0.95 \pm 0.04$ & $0.89 \pm 0.04$ & $0.91 \pm 0.04$
\end{tabular}

Table 4.4: Mean MK values and standard deviation over 25 subjects for each correction scheme. 


\subsection{Discussion}

In this study, we considered two noise correction approaches and compared their performance under different SNR in terms of reproducibility of DTI and DKI metrics, such as $F A, M D$ and $M K$, at both individual and group levels. We demonstrated the importance of these corrections for the reproducibility of the $M K$ estimation, which then becomes independent of the SNR level. Classical DTI parameters were much less influenced. This is explained by the fact that the apparent diffusion coefficient estimation is mostly based on the lowest $b$-value data points where the SNR is higher and the noise correction has a smaller effect.

\subsubsection{Comparison of the different correction schemes}

Two approaches for noise correction have been tested here, one based on the analytical expression of the first moment of the noncentral chi distribution (M1) and the other based on the second moment (M2). These corrections have been implemented with or without the non-local mean filter (BM4D). No significant differences between these methods have been detected in terms of parameters characterizing variability and reproducibility. However, by considering the number of brain voxels (including WM, GM and CSF) under the noise floor and thus forced to zero during the noise correction procedure, small differences were observed that can indicate variations in the robustness of the respective procedures. The non-local mean filter was expected to provide a more robust estimate of the first moment of the noncentral chi distribution. When applied, especially to higher $b$-value images, the number of voxels forced to zero during the M1 correction were reduced from $10 \%$ down to $2 \%$, and from $11 \%$ to $3 \%$ during the M2 correction, indicating that a number of voxels with intensity below the noise floor have been correctly assigned to a value equal or slightly above the noise floor after filtering, which was the expected effect of the BM4D filter. This was confirmed by visual inspection of filtered versus non-filtered images (not shown). This result is slightly improved with M1 as compared to M2 (2\% compared to 3\%). M2 is a straightforward and easy to implement method. However, the squared data might amplify potential errors. M1 with BM4D filtering is therefore a preferred and recommended option.

\subsubsection{MK variability}

Reported $M K$ values in the literature are very inhomogeneous. In WM, mean $M K$ values ranging from 0.51 in children (Gao et al., 2012) to 1.08 (Grinberg et al., 2011), 1 (Szczepankiewicz et al., 2013), 1.15 (Latt et al. 
2013), and 1.39 (Grossman et al., 2011) in adults, have been reported. In GM, the same studies reported MK values from 0.37 (Gao et al., 2012) to 0.73 (Grinberg et al. 2011) and 0.6 (Latt et al., 2013). Correlation of $M K$ with age for both WM and GM have been showed by Falangola et al. (2008), reporting values from 0.7 to 0.82 in GM and from 1.04 to 1.20 in WM. Noise bias correction was only applied in one of these studies (Grinberg et al., 2011). Our results after correction fall into this range, as we found WM peak for $M K=0.98$ and GM peak for $M K=0.58$ (M1+BM4D correction), for averaged data over 25 subjects. The discrepancies observed within the same age groups can be explained by the use of different approaches for image acquisition and data processing, and in particular by differences in SNR levels, which usually are not explicitly indicated. In order to reduce these differences and provide more robust and reproducible DKI estimates, including noise correction as a standard processing step, would certainly be beneficial. In particular, we have shown that two different acquisitions, with different SNRs due to increased voxel size $(2.4 \mathrm{~mm}$ isotropic for the first one and $3 \mathrm{~mm}$ isotropic for the second one) give significantly different $M K$ estimates when no correction is applied. However, this difference becomes non significant after correction. Noise correction methods, thus, allow the reproducibility and accuracy of the results at lower SNR. Acquisition at higher spatial resolution is thus clinically feasible without increasing acquisition time: partial volume effects are reduced and the chances to find small differences between two groups will increase.

The effect of noise correction was also confirmed by the ROI analysis in the selected regions, such as the Globus Pallidus. This region in particular is considered a good indicator of the SNR-related distortions in DKI analysis (Jensen and Helpern, 2010). $M K$ values in this region should be close to $M K$ values in GM. However at low SNR, the combination of the noise bias effect and the comparatively short transverse relaxation time might result in a significantly elevated $M K$ value. This "enhancement" effect was clearly demonstrated in Experiment 2 involving 25 subjects. $M K$ values in the left and right GP are extremely high when data are not corrected (respectively 1.85 and 1.76 for $\mathrm{NC}$ ) with a high inter-subject variability, and reach values close to GM areas for corrected data (respectively 0.81 and 0.81 for M1). We have shown that the noise contribution varies with the acquisition protocol and can influence the total inter-subject variability. We have also shown that this variability is spatially variable and can be influenced by the position of the head in the scanner for example. The impact of variability of DKI parameters on study design and statistical power has been studied recently by Szczepankiewicz et al. (2013). In this work, they suggested that increasing the number of subjects will reduce the variability and is more beneficial than increasing scan time to gain SNR. However, by doing so, 
the total variability might be reduced but the noise bias is still remaining, leading to erroneous estimates. Reducing variability due to noise is thus very important and noise correction is highly recommended to get better estimates and derive reliable inference in group analysis.

\subsubsection{Applicability to clinical studies}

In terms of group analysis, although only few clinical studies have been done yet with DKI, promising results have been shown. For example, DKI has proven to be a good marker for Parkinson's disease with an increase of 10 to $20 \%$ of $M K$ values in the patient group as compared to the control group in the Caudate, the Globus Pallidus, the Putamen and the Substancia Nigra (Wang et al., 2011). $M K$ has also been shown to increase with higher tumor grades (Raab et al., 2010) with a minimum of 30\% difference in MK values of different grades. Recently, DKI has been shown to be a good potential biomarker for Alzheimer's disease (Falangola et al., 2013). In animal studies, significant differences, however, no larger than $10 \%$, were reported, for example in the detection of Huntington disease (Blockx et al., 2012). Again, few of these studies included noise correction in their data processing and the SNR is not known. By decreasing the spurious inter-subject variability due to the noise bias, noise correction certainly will improve the statistical power of clinical studies, by allowing higher spatial resolution acquisition and smaller population samples.

\subsubsection{Limitations}

\section{Spatially varying noise}

The estimation of the noise standard deviation has been shown to be an important issue as it affects the subsequent noise correction procedure. Noise in SoS reconstructed magnitude images can be spatially varying as a result of the noise correlation between the channels of the receiver system (Hutton et al., 2012). In our study, the resulting spatial heterogeneity in the noise distribution had no noticeable effect due to the relatively small spatial variation of the noise standard deviation. The noise correlation was thus assumed to be of negligible impact on our noise procedure and the noise was considered spatially independent. As described in the methods section, the background noise distribution closely matches a noncentral chi distribution with a standard deviation that can be reliably estimated from Eq. 4.10. confirming the reliability of this assumption. In this work, spatially varying noise fields were thus not considered. Several factors like the use of parallel imaging and acceleration techniques Aja-Fernandez and Tristan-Vega 
2012 ) can increase the amplitude of noise spatial variations which then require more sophisticated methods of corrections (Koay and Basser, 2006 Veraart et al., 2013), already applied to DTI (Maximov et al., 2011).

\section{Physiological noise}

Another potential source of bias in diffusion imaging is the physiological noise. The main effect of this noise is the appearance of artefacts close to the ventricles, which makes the estimation of diffusion and kurtosis parameter less accurate in those areas (Kristoffersen, 2012). As modeling this type of noise and thus correcting for it a posteriori is very challenging, practical methods like cardiac gating have been shown to give better results (Chung et al. 2010, Nunes et al., 2005). However, such methods increase acquisition time which is not convenient for clinical studies. Another solution is the application of robust statistics technique adapted to the kurtosis model of diffusion to detect and remove outliers due to physiological noise (Maximov et al. 2011, 2012).

\subsection{Conclusion}

We have proposed two noise correction approaches for DW images acquired with multichannel coils, with SoS reconstruction, in the context of DKI data analysis. Our results show that noise bias correction has a strong impact on $M K$ estimation and that noise bias can lead to erroneous conclusions when conducting group studies. We demonstrated that the procedures described herein significantly reduce noise-related intra- and inter-subject variability and should not be neglected in DKI studies. Evaluation including noise correction provides accurate and reproducible results independent of the SNR and the head position. Otherwise, the final $M K$ maps are subject to biased errors depending on the spatial distribution of SNR caused both by differences in tissue relaxation and diffusion properties and, more crucially, by the spatially varying sensitivity characterizing multi-channel coils. The simplicity of the method described here allows a straightforward implementation and can be readily included in the regular pipeline for DKI analysis. Moreover, with such methods, the gain in reproducibility and accuracy of the results makes higher spatial resolution and lower SNR accessible, reducing partial volume effects in clinically feasible acquisition time. The statistical power is improved, increasing a confidence in the output results, or allowing one to reach significant conclusions with smaller population samples. 


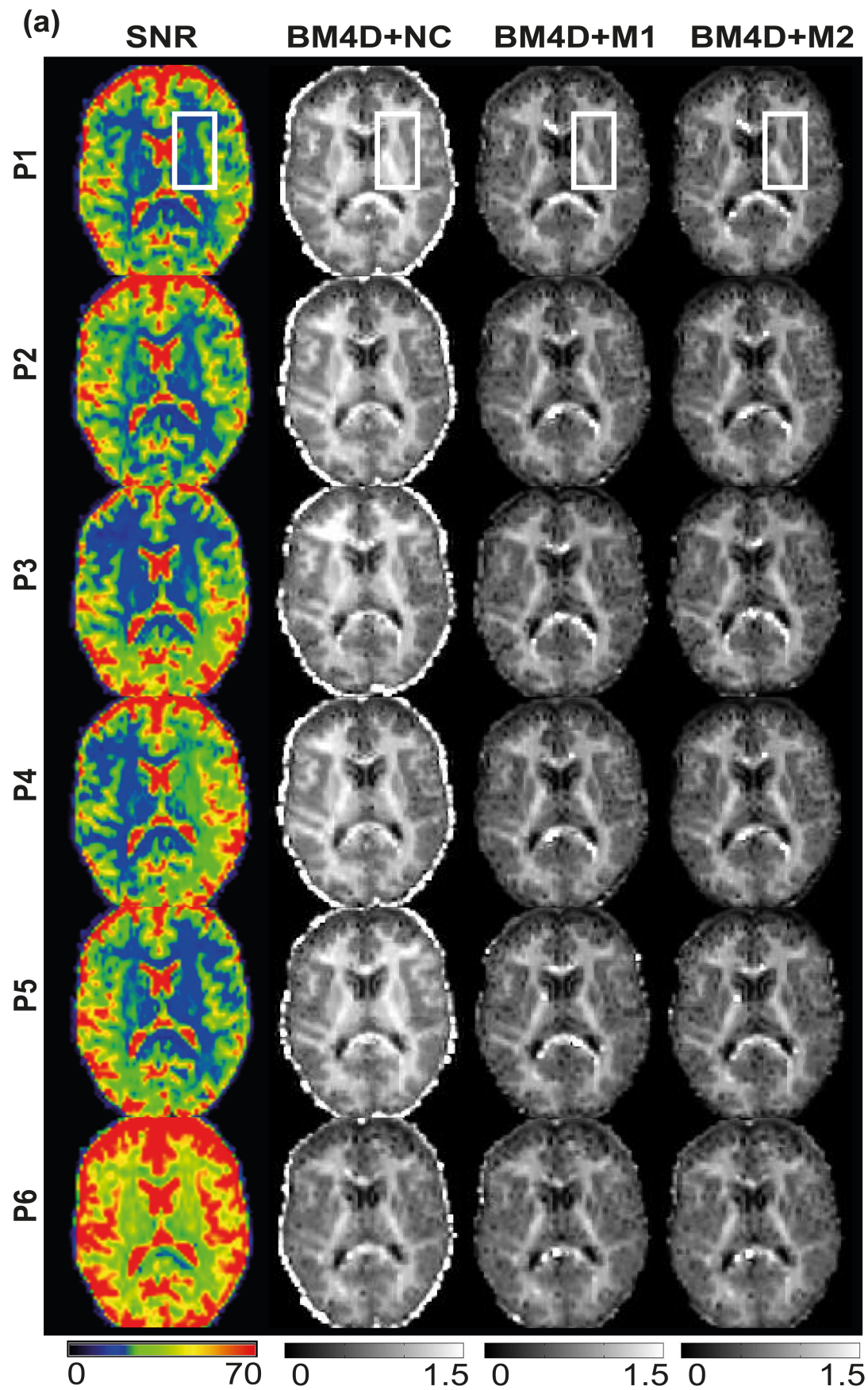

Figure 4.9: SNR of non-DWI images and corresponding MK maps for low SNR acquisitions (P1 to P5) and high SNR acquisition (P6). (a) Maps for the 6 positions and 3 different corrections. The region delineated by a white rectangle is zoomed in (b) for 4 selected positions (P1, P4, P5 and P6) and the same correction schemes (shown in color for better visualization). 


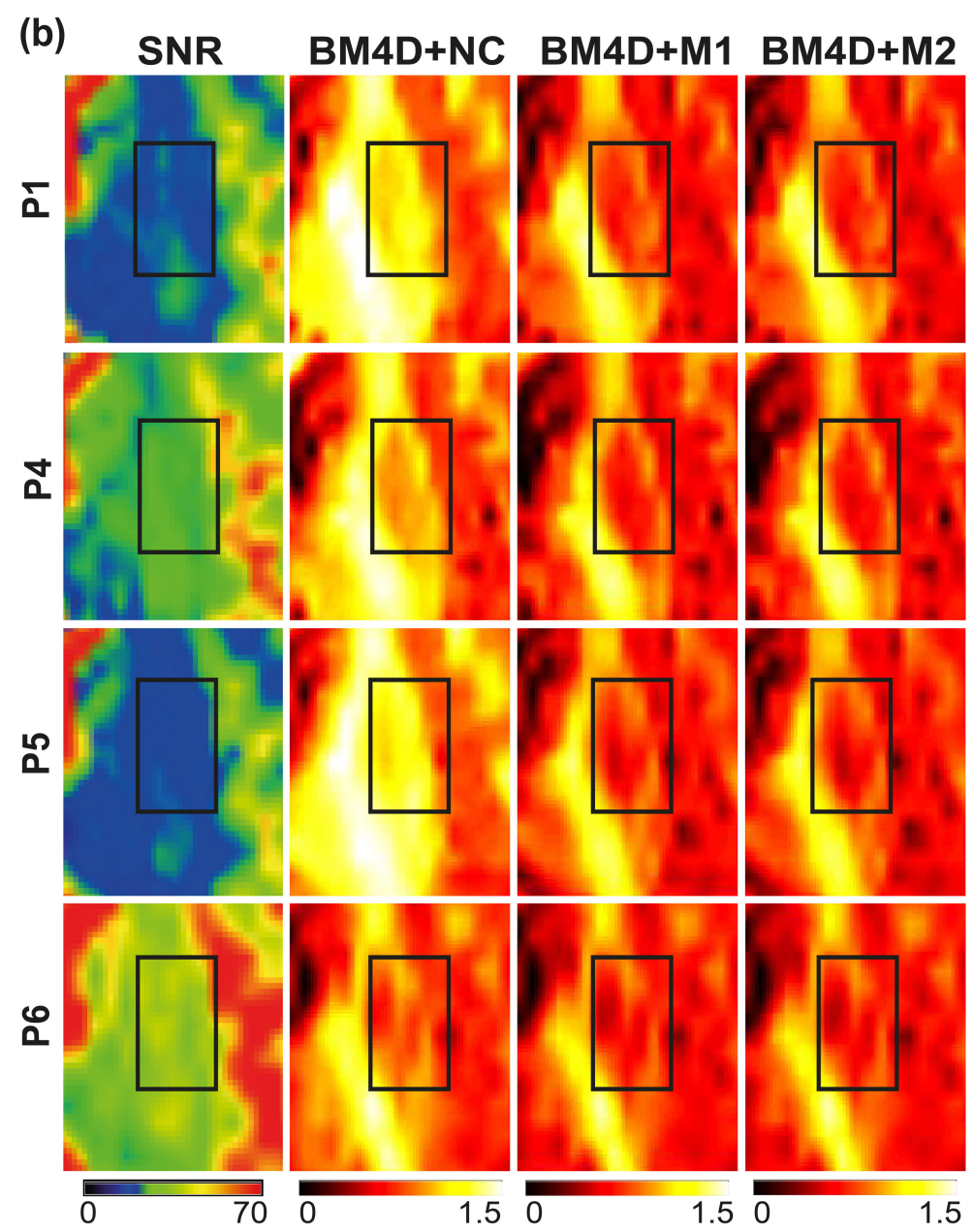

Figure 4.9: SNR of non-DWI images and corresponding MK maps for low SNR acquisitions (P1 to P5) and high SNR acquisition (P6). (a) Maps for the 6 positions and 3 different corrections. The region delineated by a white rectangle is zoomed in (b) for 4 selected positions (P1, P4, P5 and P6) and the same correction schemes (shown in color for better visualization). 

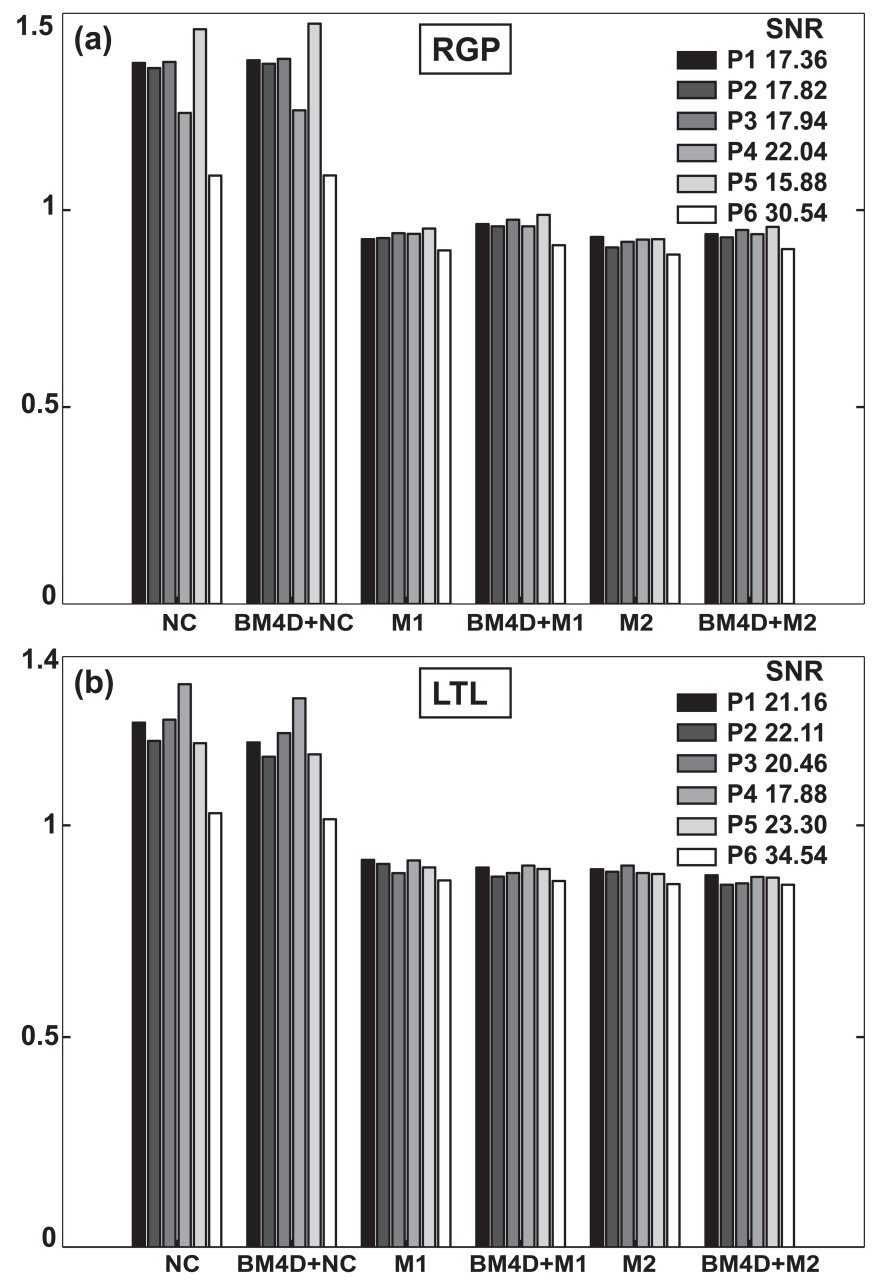

Figure 4.10: Averaged MK values for the different correction schemes in two different ROIs (a) Right Globus Pallidus and (b) Left Temporal Lobe. Results are shown for the same subject with 6 different levels of SNR corresponding to acquisitions P1 to P6. 


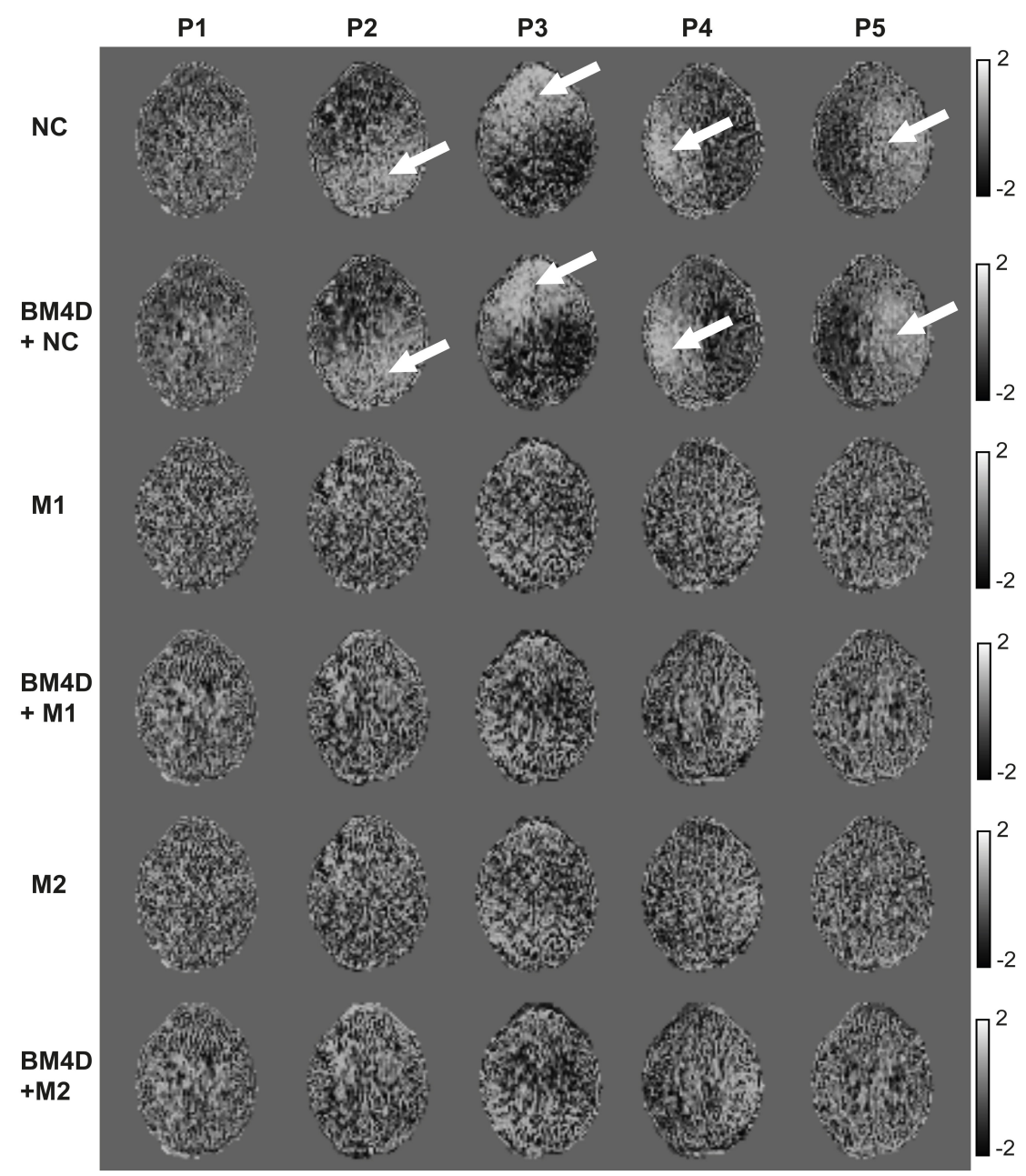

Figure 4.11: Z-score maps for each correction scheme (rows) and low SNR positions (P1 to P5). The reference is the average MK map over the 5 positions for each correction schemes. Arrows indicate regions of positive bias of $M K$ due to lower SNR. 


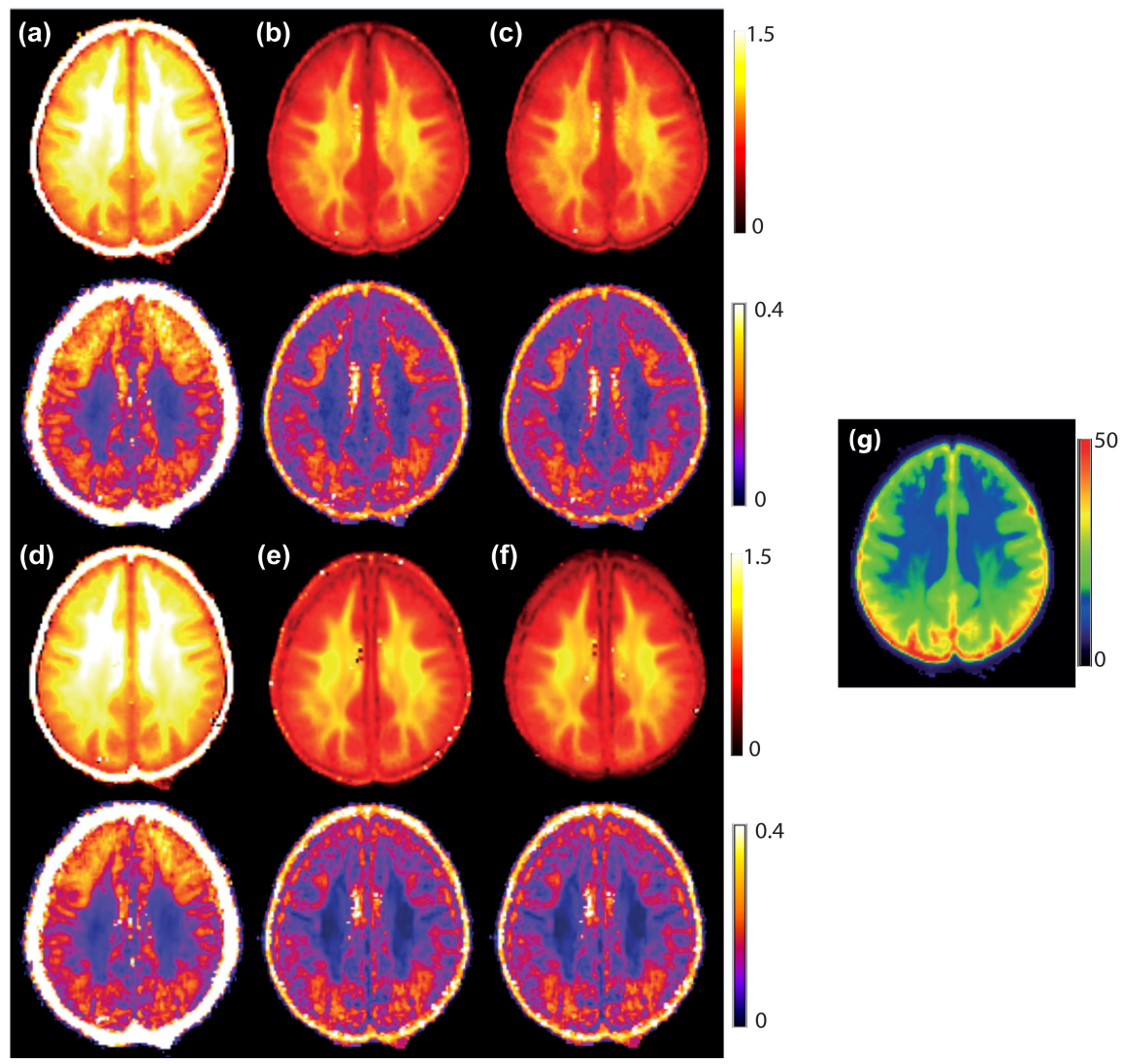

Figure 4.12: Averaged MK maps over 25 subjects for one selected slice (first and third rows) and the corresponding standard deviation maps (second and fourth rows) for (a) $N C$, (b) M1, (c) M2, (d) BM4D+NC, (e) BM4D+M1, (f) BM4D+M2. (g) Mean SNR maps of the averaged non-DW image. 

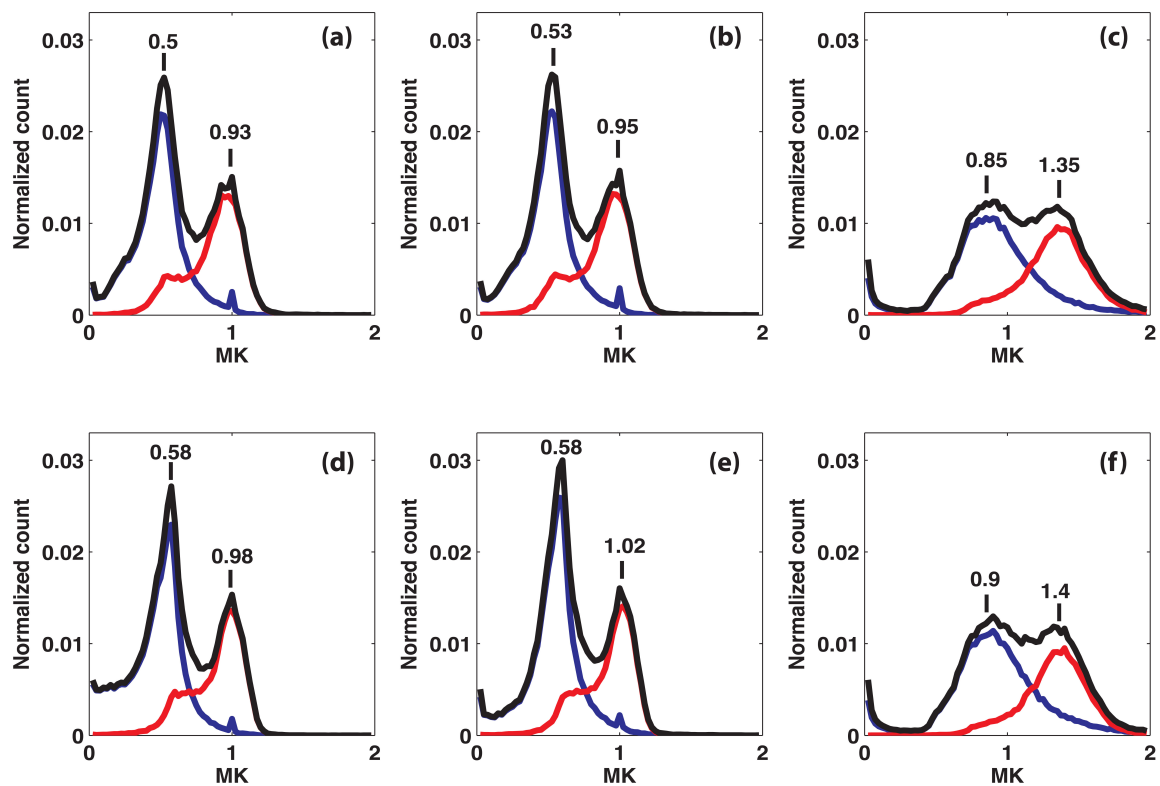

Figure 4.13: Histograms of the MK maps averaged over all subjects for all the different correction schemes for one selected slice (the same as in Figure 7): (a) M1, (b) M2, (c) NC, (d) BM4D+M1, (e) BM4D+M2, (f) $B M 4 D+N C$. Red and blue lines are the histograms for the WM and GM, respectively. 


\section{Chapter 5}

\section{Medical application to Parkinson disease}

\subsection{Introduction}

Parkinson's disease (PD), first described in 1917 by James Parkinson, is the second most common adult-onset neurodegenerative disorder after Alzheimer's disease. It mainly affects movement and may result in tremor, rigidity, bradykinesia or speech disorders. It may also result in non-motor symptoms like mood, cognitive or sleep disturbances, anosmia, amongst others. The main pathological hallmarks of the disease are the loss of dopaminergic neurons in the substantia nigra (SN), the presence of $\alpha$-synuclein containing Lewy bodies and neurites in the SN (Mhyre et al., 2012). The mention of the SN as the main locus of PD was first made by Brissaud (1899) and confirmed some years later by post-mortem studies (Tretiakoff, 1919, Foix and Nicolesco, 1925). Lewy bodies are structures located within the cytoplasm of neurons and have been shown to accumulate in the cytoplasm of dopamine neurons as the disease course increases. However, despite several major progress, PD remains a very complex disease, which is not well understood. The monitoring of the disease progression and the assessment of the treatment's efficiency is done via clinical tools, as no biomarkers have been discovered yet. The early diagnostic of PD is thus a real challenge, as the disease progression and the symptoms are extremely heterogeneous from a patient to an other.

The recent progresses in neuroimaging are promising for PD characterization to get more accurate diagnosis (Stoessl et al., 2011). In particular, strucutural MRI has been shown to be a good tool to make a difference between several types of PD: multiple system atrophy, progressive supranuclear palsy, corticobasal degeneration or neurodegeneration NBIA1 Mhyre 
et al. 2012). The main locus of the disease is the SN, more precisely in the pars compacta of the $\mathrm{SN}$ (SNpc), which is a very small region located in the midbrain. At the onset of the motor symptoms of the disease, approximately $60-70 \%$ of dopamine neurons in the SN are already lost (Mulkern et al., 2009). It is thus the targeted region for MRI studies. As the disease progresses, more regions, mainly located in the basal ganglia are also affected. These regions are the caudate, the putamen, the globus pallidus, the ventral part of the thalamus, amongst others. However there is no neuronal loss in these regions but rather a loss of dopaminergic terminals, which is rather difficult to detect.

Diffusion MRI has also been proposed as a potential way to diagnose the disease or to measure the effect of a treatment. FA has been shown to correlate negatively with the number of neurons in the $\mathrm{SN}$ in a mouse model of PD (Boska et al., 2007). Similar results could be expected in humans, but currently the results are quite heterogeneous as described in a recent meta-analysis (Cochrane and Ebmeier, 2013). The lack of reproducible results could be explained by the differences of protocols (number of diffusion directions, image resolution), processing (ROI analysis or voxel-based analysis), number of patients, disease severity, medication intake, etc.

Recently a new diffusion method, DKI, was proposed (Jensen and Helpern, 2010). One can refer to the introduction of this thesis (section 2.4.3) for more detailed explanation. One advantage of applying this method to PD is the increased sensitivity of the derived kurtosis parameters to microstructure organisation in GM. Until now, only two studies have published results showing differences in kurtosis parameters in a group of PD patients. Increase of MK in the group of patients in both putamen and the SN was reported by Wang et al. (2011). Kamagata et al. (2013) investigated the alteration of cingulate fibers in patients with PD. MK and FA in the anterior cingulum were significantly lower in PD patients than in healthy controls and the diagnostic performance of MK compared to FA was higher.

In this chapter, we will study a population of 27 early-stage PD patients with 26 age and sex-matched controls using DKI and compare the local values of kurtosis metrics in a voxel-based analysis (VBA). Previous methods described in chapter 3 and 5 will be applied to improve accuracy of the results.

\subsection{Methods}

\subsubsection{Population}

A total of 27 (14 males; mean age: $65.6 \pm 7.5)$ patients clinically diagnosed with Parkinson's disease and 26 (14 males; mean age: $64.4 \pm 7.8$ ) healthy 
control subjects were recruited. The groups were matched by age, sex, and highest achieved education level. Patients went through a number of tests to assess the disease stage and severity. For this purpose, three classical tests were used. The Hoehn and Yahr scale (Hoehn and Yahr, 1967) was used to assess the stage of the disease, which scales from 1, unilateral disease to 5, wheelchair/bed bound. The Unified Parkinson's Disease Rating Scale (UPDRS) (Fahn and Elton, 1987) was used to investigate the motor symptoms severity: in addition to the motor symptoms, behavior, mood and daily life of the patient were evaluated giving a bigger picture of the effects of the disease. The 39-question Parkinson's Disease Questionnaire (PDQ39) (Jenkinson et al., 1997), which assesses quality of life was also used.

In addition, subjects were also tested to evaluate various aspects of their psychological state: global cognitive function [Mattis Dementia Rating Scale (Mattis, 1988), MMSE (Mini Mental State Examination) (Folstein et al. 1975), Symbol Digit Modalities test, verbal fluency test], inhibition [Stroop test, random number generation (Jahanshahi et al., 2006)], episodic memory [Rey auditory verbal learning test (Rey, 1964), updating of working memory [letter running span memory task], cognitive flexibility [Modified Wisconsin Card Sorting Test], visuospatial judgment [Judgment of Line Orientation (Benton et al., 1978)], and anxiety [Hospital Anxiety and Depression Scale (Zigmond and Snaith, 1983)].

Twenty-three of the 27 patients were taking a combination of several classes of drugs: levodopa (immediate and controlled release), nonergot-derived dopamine receptor agonists (Pramipexole, Ropinirole), and a monoamine oxidase B inhibitor (Rasagiline). The remaining 4 patients were not taking antiparkinsonian medication at the time of scanning. Levodopa and dopamine agonist dosages were pooled and summarized as the equivalent daily dosage of levodopa [LEDD, (Hobson et al., 2002)]. Total daily L-dopa equivalent dosages ranged from 0 to $900 \mathrm{mg}$. A summary of the study cohort can be found in Table 1. Written informed consent was obtained from all participants in accordance with the Declaration of Helsinki. The Ethics Committee of the University of Liège approved the study.

\subsubsection{Data processing}

\section{Acquisition protocol}

Diffusion data were acquired with a 3T head-only scanner (Magnetom Allegra, Siemens) using a twice refocused spin-echo diffusion sequence. Two sets of 120 DW images with non-collinear diffusion encoding directions were acquired with respectively a $b$-value of $1000 \mathrm{~s} / \mathrm{mm}^{2}$ and a $b$-value of 2500 
$\mathrm{s} / \mathrm{mm}^{2}$. In addition 22 non-DW images were interleaved with the DW ones for motion correction (see Chapter 3). Other parameters were set to: $\mathrm{TR} / \mathrm{TE}=6800 / 91 \mathrm{~ms}$, matrix size : $88 \times 88 \times 54$. Voxels were $2.4 \mathrm{~mm}$ isotropic.

For both display purposes and calculation of the intracranial volume, a multi-parameter protocol based on a 3D multi-echo fast low angle shot (FLASH) sequence (Weiskopf and Helms, 2008) was also used to calculate structural and quantitative maps of $\mathrm{T} 1, \mathrm{~T} 2^{*}$, proton density $(\mathrm{PD})$ and magnetization transfer $(\mathrm{MT})$ at $1 \times 1 \times 1 \mathrm{~mm}^{3}$ resolution. Three co-localized $3 \mathrm{D}$ multi-echo FLASH datasets were acquired with predominantly proton density weighting ( $\mathrm{PDw}$ : $\mathrm{TR} / \alpha=23.7 \mathrm{~ms} / 6^{\circ}$ ), $\mathrm{T} 1$ weighting ( $\mathrm{T} 1 \mathrm{w}: 18.7$ $\mathrm{ms} / 20^{\circ}$ ), and MT weighting (MTw: $23.7 \mathrm{~ms} / 6^{\circ}$; excitation preceded by an off-resonance Gaussian MT pulse of $4 \mathrm{~ms}$ duration, $220^{\circ}$ nominal flip angle, $2 \mathrm{kHz}$ frequency offset). Volumes were acquired in 176 sagittal slices using a $256 \times 224$ voxel matrix. A B1 map was calculated using the actualflip-angle imaging method (Yarnykh, 2007) based on two interleaved 3D FLASH acquisitions (repetition times TR $1=33 \mathrm{~ms}$, TR2 $=165 \mathrm{~ms}$, nominal flip angle $=60^{\circ}$, acquisition time $=5 \mathrm{~min}$.). The B1 map was used to correct the multi-parameter maps for B1 field bias (Volz et al., 2010).

\section{Preprocessing}

DW images were first realigned for motion using the interpolation method as described in Chapter 3 . Non-DW images were coregistered to the first image and movements were interpolated in-between and applied to each individual DW image. Diffusion directions were rotated accordingly. The iterative procedure described in Chapter 3 could not be applied here, as it is not adapted for high $b$-value acquisition, where signal decay is no more monoexponential. Then, BM4D filtering (Maggioni et al., 2013) was applied and images were corrected for the noise bias using the method M2 developed in Chapter 4

\section{Kurtosis tensor estimation}

The kurtosis tensors were then estimated using ExploreDTI (Leemans et al. 2009). A non-linear least square approach with outlier detection has been applied to directly, simultaneously, and robustly estimate the diffusion and kurtosis tensors (Veraart et al., 2013). FA, MK, AK and RK parameter maps were derived from the tensors using the equations described in section 2.4 .3 . 


\section{Inter-subject spatial normalization}

In order to compare data, FA maps were spatially normalized using the method described in FSL (Smith et al. 2004) for TBSS (Tract-Based Spatial Statistics (Smith et al., 2006)) analysis. The FA template was transformed to be symmetrical by processing the averaged between the template and its flipped version. Same transformations were applied to MD, MK, AK and RK maps. In addition data of patients affected mainly on the left side were left-right flipped to have the main affected hemishere on the left for all the patients.

\section{Processing of structural images}

Magnetization transfer (MTsat) saturation maps were calculated within SPM8 (Draganski et al., 2011) and processed using unified segmentation in order to create masks of GM, WM, and CSF (Ashburner and Friston 2005). The total intracranial volume (ICV) in cubic millimeters was calculated for each subject using the segmented volumes. Diffeomorphic registration (DARTEL) was used to bring the MTsat maps into MNI space Ashburner, 2007). Intensity modulation of the MTsat maps was performed using a combined weighting and smoothing procedure (Draganski et al., 2011). The MTsat images of the 10 patients with left-side dominant symptoms were also left-right mirrored. Finally, they were averaged to create a study-specific anatomical template on which to overlay the statistical maps.

\subsubsection{Statistical analysis}

Statistical analysis of scalar images (FA, MD, MK, RK and AK) were performed using SPM8 with parametric methods (Friston et al., 2007), using two-sample $t$-test to compare the two groups. To insure that remaining variance due to age or total intracranial volume is taken into account, age and ICV were added as nuisance covariates: regression were thus set up for all the subjects together as no interaction between the covariates and the groups were assumed. No additional smoothing was applied to the images, the voxels in normalized images are $1 \mathrm{~mm}^{3}$ isotropic and the measured smoothness was $3.7 \mathrm{~mm} \times 4.7 \mathrm{~mm} \times 4.4 \mathrm{~mm}$ for MK maps and of the same order for the other parameters. Unequal variance was assumed between the two groups. Cluster-level familywise error rate corrected pvalues $\left(p_{\mathrm{FWE}}<0.05\right)$ were used to identify regions of significant difference between groups. A single-voxel uncorrected height threshold of $p<0.001$ was used for cluster forming and exploration of the data.

An additional small volume correction analysis was performed for regions previously mentioned in the literature for DKI in the case of PD Wang 
et al., 2011, Kamagata et al., 2013). These regions include: the brainstem, the globus pallidus, the caudate, the putamen and the cingulate. The same statistical analysis were performed using these regions as an explicit mask in the analysis. The regions were delineated using the Harvard-Oxford subcortical structural atlas and the JHU white-matter tractography atlas available in FSL (Analysis Group, FMRIB, Oxford, UK (Jenkinson et al., 2012)).

\subsection{Results}

\subsubsection{Cognitive evaluation}

In our cohort the Parkinson's disease patients scored significantly lower than controls on the Mattis scale, mini-mental state examination, and the episodic memory test (Rey auditory verbal learning test), suggesting mild cognitive disturbances. Apart from these, there were no statistically significant differences between the patients and controls on any of the psychological assessments. Every patient's performance was above the standard cutoff threshold for the Mattis dementia rating scale (Llebaria et al., 2008), and none of them met standard criteria for dementia associated with Parkinson's disease. A summary of the test results as well as general information about the groups is presented in table 5.1 .

\subsubsection{Voxel based analysis of diffusion parameters}

Diffusion tensor metrics: MD and FA

No significant differences were found in MD maps. Group differences in FA were found in two clusters: healthy controls had significantly higher FA than patients in both the right amygdala and the posterior thalamic radiation. These results are summarized in Table 5.2 and are shown in Figure 5.1 .

\section{Kurtosis metrics: MK, RK and AK}

In total, 9 regions were found significantly different for at least one of these metrics: MK, RK or AK. In particular the superior parietal lobule was affected for the three parameters. Only one overlap with FA difference was found in the right amygdala where AK is significantly higher in patients $\left(p_{F W E}=0.025\right)$. Other regions include the precentral gyrus, uncinate fasciculus, the post central gyrus, the premotor cortex and the parietal lobe. The results are summarized in table 5.3 for MK, in table 5.4 for AK and in table 5.5 for RK. Clusters can be visualize on Figure 5.1. 


Age
Sex (M:F)
Years of Education
ICV (mm3)
BMI (Kg/m2)
Hand Dominance (L:R)
Hoehn \& Yahr stage
Most Affected Side (L:R)
Disease Duration (years)
LEDD (mg)
UPDRS Section 2
UPDRS Section 3
Mattis
MMSE
HADS total
PDQ39 Mobility
PDQ39 Total
RAVLT
SDMT
JOLO

$\begin{array}{ccc}\text { Healthy controls } & \text { Parkinson's patients } & \text { t-test p-value } \\ 64(8) & 66(8) & 0.549 \\ 14: 12 & 14: 13 & \\ 13(3) & 11(3) & 0.133 \\ 1478(152) & 1516(148) & 0.360 \\ 25(3) & 25(3) & 0.660 \\ 2: 24 & 2: 25 & \\ & 1.5(0.62) & \\ & 10: 17 & \\ & 5(3) & \\ & 323(255) & \\ & 9(6) & 0.004 \\ & 14(7) & 0.022 \\ 139(4) & 136(4) & 0.066 \\ 29(1) & 28(1) & \\ 10(4) & 13(6) & 0.006 \\ & 20(18) & 0.062 \\ 53(11) & 189(114) & 0.055 \\ 51(10) & 44(11) & \\ 27(4) & 45(12) & \end{array}$

Table 5.1: Test results and information about the groups of patients and healthy controls. Values reflect mean (standard deviation). Two-tail t-tests were performed with an assumption of unequal variance in each group. ICV = Intracranial Volume, BMI = Body Mass Index, LEDD $=L-D O P A$ Equivalent daily dose (Hobson et al., 2002), UPDRS = Unified Parkinson's Disease Rating Scale, MMSE = Mini Mental State Examination, HADS = Hospital Anxiety and Depression Scale, PDQ = Parkinson's Disease Questionnaire, RAVLT=Rey Auditory Verbal Learning Test, SDMT = Symbol Digit Modalities Test, JOLO=Judgment of line orientation test

\begin{tabular}{cccccl} 
pFwe & Size $\left(\mathbf{m m}^{\mathbf{3}}\right)$ & \multicolumn{3}{c}{ Peak voxel } & Labels \\
& & $\mathbf{x}(\mathbf{m m})$ & $\mathbf{y}(\mathbf{m m})$ & $\mathbf{z}(\mathbf{m m})$ & \\
0.045 & 114 & 31 & 0 & -17 & Right amygdala \\
0.007 & 154 & 34 & -65 & 9 & $\begin{array}{l}\text { Posterior thalamic radiation } \\
\text { (optic radiation) }\end{array}$
\end{tabular}

Table 5.2: Table of significant clusters where FA is larger in controls

\section{Small volume correction}

Small volume corrections were performed in 5 regions: brainstem, pallidus, cadate, putamen and cingulate, using masks. FA was found higher in the brainstem in controls. MK, RK and AK were found higher in the putamen in patients. These results are summarize in Table 5.6. Figure 5.2 shows both clusters in the brainstem and in the putamen. 


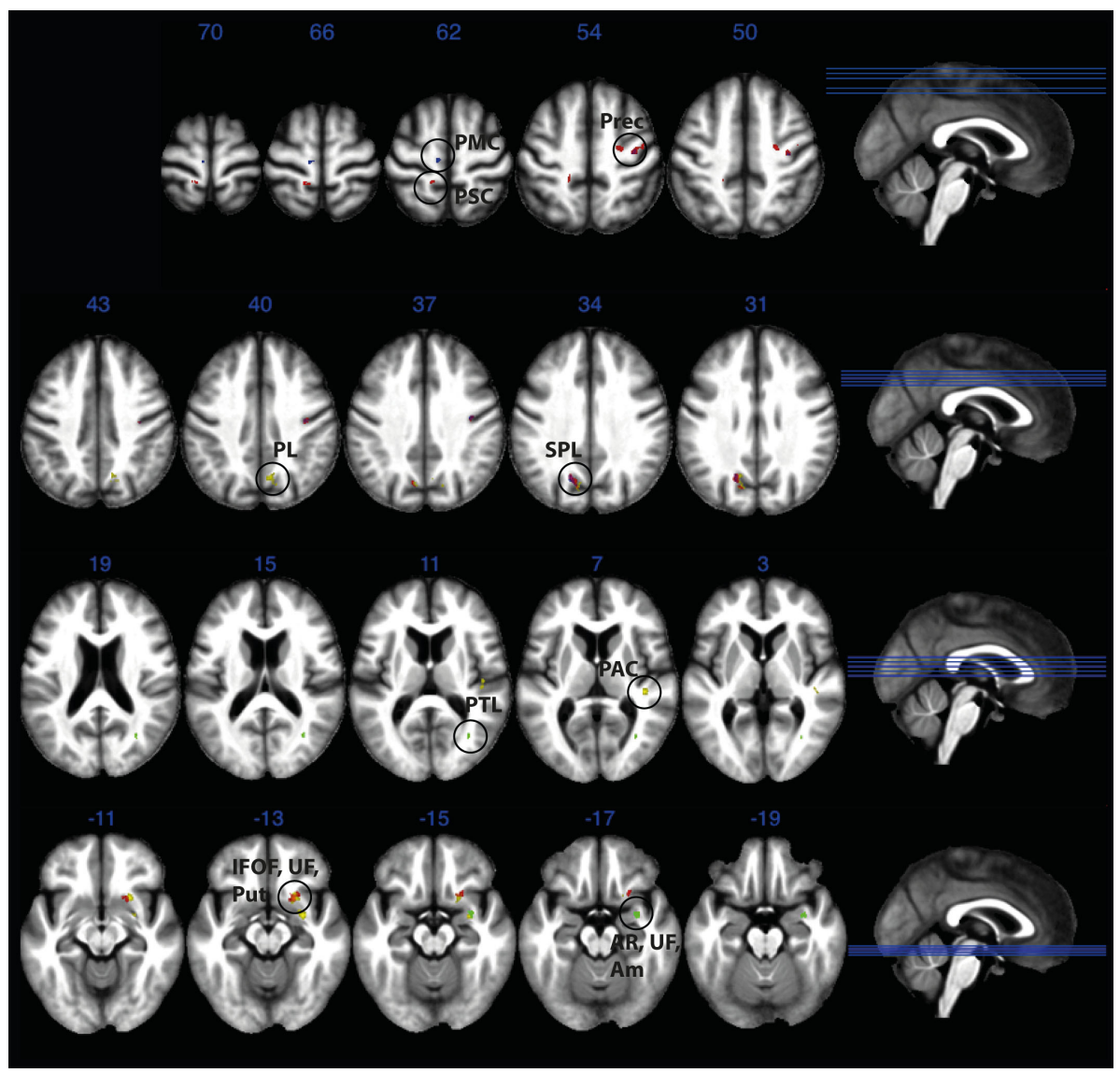

Figure 5.1: Significant clusters for all the parameters are displayed on the mean structural MTsat image. FA (green) is lower in patients in a region at the boundaries of the right amygdala (Am), the uncinate fasciculus (UF) and the acoustic radiation $(A R)$ and in the right Posterior thalamic radiation (PTL); $M K$ (red), RK (blue) and $A K$ (yellow) are higher in patients than in controls in the same region where FA is lower ( $A m, U F, A R)$, in a region encompassing the right Inferior fronto-occipital fasciculus (IFOF), the UF and a small portion of the putamen (Put), in the right primary auditory cortex (PAC), the left superior parietal lobule (SPL), the right parietal lobe $(P L)$, the right precuneus (Prec), the left primary somatosensory cortex $(P S C)$ and the left primary motor cortex (PMC).

\subsection{Discussion}

In this voxel-based analysis of kurtosis metrics, we found several group differences in the cerebrum. Most of the clusters were in regions known for 


\begin{tabular}{|c|c|c|c|c|c|}
\hline \multirow[t]{2}{*}{ pFWE } & \multirow[t]{2}{*}{$\operatorname{Size}\left(\mathrm{mm}^{3}\right)$} & \multicolumn{3}{|c|}{ Peak voxel } & \multirow[t]{2}{*}{ Labels } \\
\hline & & $\mathbf{x}(\mathbf{m m})$ & $\mathrm{y}(\mathbf{m m})$ & $\mathbf{z}(\mathbf{m m})$ & \\
\hline$<0.001$ & 288 & -16 & -62 & 32 & $\begin{array}{l}\text { Superior parietal lobule } \\
\text { Precuneus }\end{array}$ \\
\hline$<0.001$ & 275 & 38 & -11 & 53 & $\begin{array}{l}\text { Precentral gyrus } \\
\text { Primary motor cortex }\end{array}$ \\
\hline 0.038 & 142 & 24 & -10 & 52 & $\begin{array}{l}\text { Precentral gyrus } \\
\text { Primary motor cortex }\end{array}$ \\
\hline 0.002 & 227 & 22 & 13 & -13 & $\begin{array}{l}\text { Inferior fronto-occipital fasciculus } \\
\text { Uncinate fasciculus } \\
\text { Putamen }\end{array}$ \\
\hline 0.007 & 194 & -14 & -40 & 65 & $\begin{array}{l}\text { Primary somatosensory cortex } \\
\text { Primary motor cortex } \\
\text { Superior parietal lobule }\end{array}$ \\
\hline
\end{tabular}

Table 5.3: Table of significant clusters where $M K$ is larger in patients

\begin{tabular}{|c|c|c|c|c|c|}
\hline \multirow[t]{2}{*}{ pFWE } & \multirow[t]{2}{*}{$\operatorname{Size}\left(\mathrm{mm}^{3}\right)$} & \multicolumn{3}{|c|}{ Peak voxel } & \multirow[t]{2}{*}{ Labels } \\
\hline & & $\mathrm{x}(\mathbf{m m})$ & $\mathrm{y}(\mathrm{mm})$ & $\mathbf{z}(\mathbf{m m})$ & \\
\hline$<0.001$ & 236 & 24 & 14 & -12 & $\begin{array}{l}\text { Inferior fronto-occipital fasciculus } \\
\text { Uncinate fasciculus } \\
\text { Putamen }\end{array}$ \\
\hline 0.001 & 215 & 41 & -28 & 8 & $\begin{array}{l}\text { Right Primary auditory cortex } \\
\text { Acoustic radiation }\end{array}$ \\
\hline 0.025 & 129 & 30 & -2 & -14 & Right amygdala \\
\hline 0.001 & 197 & -14 & -61 & 29 & $\begin{array}{l}\text { Superior parietal lobule } \\
\text { Precuneus }\end{array}$ \\
\hline$<0.001$ & 228 & 9 & -63 & 40 & Parietal lobe \\
\hline
\end{tabular}

Table 5.4: Table of significant clusters where AK is larger in controls

\begin{tabular}{cccccl} 
pFWE & Size $\left(\mathbf{m m}^{\mathbf{3}}\right)$ & \multicolumn{3}{c}{ Peak voxel } & Labels \\
0.004 & 181 & -16 & -65 & 33 & $\begin{array}{l}\text { Superior parietal lobule } \\
\mathbf{x}(\mathbf{m m}) 7 \mathrm{~A}, 7 \mathrm{M})\end{array}$ \\
0.013 & 152 & 39 & -15 & 39 & $\begin{array}{l}\text { Precentral gyrus } \\
\mathbf{y}(\mathbf{m m})\end{array}$ \\
0.037 & 128 & -5 & -23 & 68 & $\begin{array}{l}\text { Primary motor cortex } \\
\text { Premotor cortex }\end{array}$
\end{tabular}

Table 5.5: Table of significant clusters where $R K$ is larger in controls

their role in motor functions. In particular the superior parietal lobule was quite affected (increase of the parameters in patients: MK, RK and AK). Another region was the amygdala, which is usually more involved in the case of PD with dementia. However, the neuropsychological testing did indicate very mild cognitive disturbances in the patients, which could explain this finding. Differences were also found in the auditory cortex. However no differences were found in the expected regions: the nigrostriatal pathway and 


\begin{tabular}{llccccc} 
Region & Parameter & pFwE & Size $\left(\mathbf{m m}^{\mathbf{3}}\right)$ & \multicolumn{3}{c}{ Peak voxel } \\
& & & & $\mathbf{x}(\mathbf{m m})$ & $\mathbf{y}(\mathbf{m m})$ & $\mathbf{z}(\mathbf{m m})$ \\
Putamen & MK (P>C) & 0.022 & 79 & 16 & 9 & -7 \\
Putamen & RK (P>C) & 0.044 & 49 & 14 & 13 & -8 \\
Putamen & AK (P>C) & 0.019 & 71 & 16 & 8 & -8 \\
Brainstem & FA (C>P) & 0.004 & 71 & -9 & -32 & -37
\end{tabular}

Table 5.6: Table of significant clusters using region-of-interest masking. $P$ stands for patients and $C$ for controls
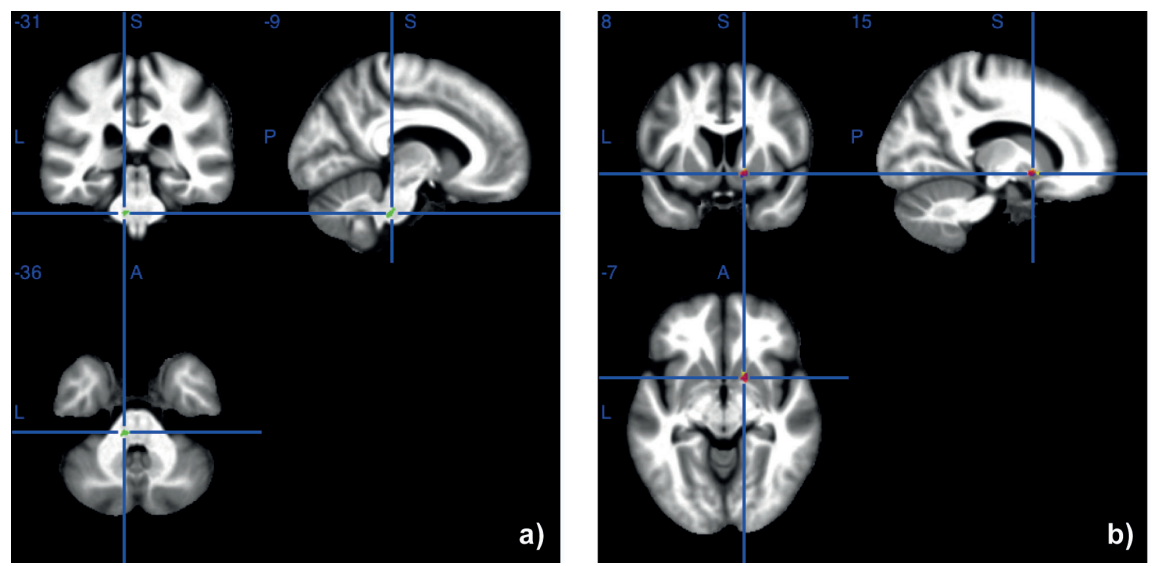

Figure 5.2: Significant clusters after small volume correction. a) FA is increased in controls in the brainstem and $M K, R K$ and $A K$ are increased in patients in the putamen.

the brainstem, except for a small cluster at the limit between the putamen (GM) and the inferior fronto-occipital and uncinate fasciculus (WM), where FA was found lower in patients $\left(p_{F W E}=0.045\right)$ and AK was found significantly higher $\left(p_{F W E}=0.025\right)$. Small volume corrections in specific ROIs confirmed a change in the putamen. However, these clusters were found in the right hemisphere, while we would have expected results in the left hemisphere, contralateral to the most affected body side. A small cluster of decreased FA in patients was also found in the brainstem, however it was in the lowest slices and could be due to the presence of image distortions. Higher MK in PD patients was reported before in the SN and the putamen (Wang et al., 2011) and lower MK values in the anterior cingulum has been reported by another group Kamagata et al. (2013) : none of these results was replicated in our case, except for a small cluster in the putamen after small volume correction. The discrepancies could be due to protocol differences and to the choice of ROI analysis compared to a voxel-based analysis. 
The interpretation of diffusion metrics in general and kurtosis ones in particular is still not clear. These parameters can be affected by a number of biological factors such as axonal damages, presence/absence of myelin, presence of different cells, etc. In our case, most of the clusters for kurtosis parameters were found close to a GM/WM limit, while for FA, they were all in the WM. Increases in kurtosis are usually associated with an increase of restriction and diffusion complexity. For example if axons are damaged, the diffusion along the fiber will be less fluid, leading to an increase of kurtosis along the main diffusion direction (AK) and thus also an increase of MK. In the case of $\mathrm{PD}$, it has been shown that axonal degeneration may be the first feature of PD, before the loss fo neuronal somata in the SN (Burke and K. 2013). Increased mean kurtosis may be then associated with this axonal degeneration.

This study confirms that kurtosis parameters are more sensitive to changes than FA or at least provide a complementary insight into tissue microstructure. Therefore these parameters would represent better candidates for a potential biomarker. However, the same data, analysed with another new approach called track density imaging in which the manufactured signal is simply the number of fibers passing through each voxel (Calamante et al. 2010) demonstrated better sensitivity. Similar cortical regions were found different in patients (superior parietal lobule, right amygdala, auditory cortex, primary somatesensory cortex) and in an interesting fashion these clusters were found in the WM just next to our clusters, which are partly in the GM at the end of the tracks. Moreover, the most interesting finding was in the nigrostriatal pathway, showing more sensitivity in that region than kurtosis metrics (Ziegler et al., 2014).

\subsection{Conclusion}

In this chapter, we have demonstrated how DKI can be applied in clinical research to investigate potential biomarkers of PD. At this stage, the results are quite promising and further studies are needed to confirm our findings. In particular, no significant difference was found in sub-cortical areas like the SN. In the future, more subjects will be added to the existing pool. A ROI analysis with more specific maps could be applied using MT maps for the delineation of regions, especially for SN. However towards this end, a more robust normalization should be used to make sure parameters maps and MT maps are well registered. Multivariate approaches for pattern recognition would also be an interesting method to investigate more deeply the potential of DKI for diagnosis. 


\section{Chapter 6}

\section{Conclusion and perspectives}

The first in vivo diffusion imaging of the human brain dates back to 25 years ago and huge progress has been made since then. However, as the field is developing, there are still no standard accepted methods to process this type of data. In particular, the number of methods to analyse diffusion data at high $b$-values ( $2000 \mathrm{~s} / \mathrm{mm}^{2}$ and above) has increased considerably but the basic processing steps, such as motion correction have not been adapted. The main focus of this thesis was thus to provide adapted methods for both motion and noise correction and to apply diffusion kurtosis imaging in clinical studies.

Chapter 3 was dedicated to the investigation of a new method based on an iterative procedure to coregister each individual DW image to its theoretical pair, using the DTI model. The quality of the coregistration is improved by matching identical intensity distributions. The main drawback of this method being its high computational time, we have also shown a low computational cost alternative consists in coregistration of interleaved non-DW images followed by interpolation of the transformations for DW images. Furthermore, this method does not depend on a particular model and can be applied to any $b$-value, as far as non-DW images are acquired every few DW images. In this work, we have chosen to investigate only motion, and thus rigid-body tranformation were used. However, motion correction is often combined with eddy current corrections by using a full affine transformation (adding shear and scale). As coregistration performs quite poorly with high $b$-values due to the lack of strong boundaries between tissue and very different contrasts between low and high $b$-value images, an alternative method should be developed.

In chapter 4, another important aspect of data quality was investigated. 
MRI is a low SNR technique and diffusion MRI has even lower SNR as it focuses on the signal decay. Through this work, one of the main goal was to deliver methods that are easily interfaced in order to make them easily usable for people with non-technical background. The methods we have proposed for noise correction meet this criterion and have been successfully applied in ongoing studies. However, these methods are based on noncentral $\chi$ distribution, which is one of their main limits. Nowadays, parallel imaging using GRAPPA or SENSE reconstruction with acceleration factors becomes more and more standard. The signal distribution is much more complex in these cases and spatially varying noise has to be considered.

In chapter 5, Parkinson disease was investigated with DKI and methods described in Chapter 3 and 4 were used. Significant results were found in several cortical areas, giving promising results.

This work combine both methodological aspects and application, tackles several issues related to diffusion data quality and opens avenues for fugure work.

As mentioned earlier, diffusion imaging acquisitions are still quite long and volunteers or patients might move significantly. Therefore, it is important to apply iterative motion correction to high $b$-value images in general. Moreover, one should add distortion correction for eddy current to the current set-up. Another possible improvement would be to use more robust tensor estimation, to make sure tensors are positive definite.

Concerning the chapter about noise correction, the first step will be to consider more general cases, in particular to investigate methods for correcting spatially variable noise. For this purpose, MRI phantoms (e.g. polymers) or simulations are suitable.

Finally, for the Parkinson's disease study, more subjects will be added to the current study. From a methodological point of view, spatial normalization should be improved to be able to do a region-of-interest analysis based on MT sequence. Multivariate pattern analysis should also be investigated to assess the diagnostic potential of mean kurtosis and related metrics. 


\section{Abbreviations}

$\begin{array}{ll}\text { ADC } & \text { Apparent diffusion coefficient } \\ \text { AK } & \text { Axial kurtosis } \\ \text { BMI } & \text { Body mass idex } \\ \text { CNR } & \text { Contrast-to-noise ratio } \\ \text { CSF } & \text { Cerebro spinal fluid } \\ \text { DKI } & \text { Diffusion kurtosis imaging } \\ \text { DTI } & \text { Diffusion tensor imaging } \\ \text { DW } & \text { Diffusion weighted } \\ \text { EPI } & \text { Echo planar imaging } \\ \text { FA } & \text { Fractional anisotropy } \\ \text { FOV } & \text { Field of view } \\ \text { GM } & \text { Grey matter } \\ \text { GP } & \text { Globus pallisdus } \\ \text { GRAPPA } & \text { Generalized autocalibrating partially parallel acquisition } \\ \text { HADS } & \text { Hospital Anxiety and Depression Scale } \\ \text { ICV } & \text { Intracranial volume } \\ \text { IPR } & \text { Iterative procedure for realignment } \\ \text { JOLO } & \text { Judgment of line orientation } \\ \text { LEDD } & \text { L-Dopa equivalent daily dose } \\ \text { MD } & \text { Mean diffusivity } \\ \text { MI } & \text { Mutual information } \\ \text { MK } & \text { Mean kurtosis } \\ \text { MMSE } & \text { Mini Mental State Examination } \\ \text { MNI } & \text { Montreal Neurological Institute } \\ \text { MR } & \text { Magnetic resonance } \\ \text { MRI } & \text { Magnetic resonance imaging } \\ \text { MT } & \text { Magnetization transfer } \\ \text { PD } & \text { Parkison's disease } \\ \text { PDF } & \text { Probability distribution function } \\ \text { PDQ39 } & \text { 39-question Parkinson's Diesase Questionnaire } \\ \text { PDw } & \text { Proton density weighting } \\ & \end{array}$


PGSP Pulsed gradient spin echo

RAVLT Rey Auditory Verbal Learning Test

RF Radio-frequency

RK Radial kurtosis

RMSE Root mean square error

ROI Region of interest

SDMT Symbol Digit Modalities Test

SENSE Sensitivity encoding

SNR Signal-to-noise ratio

SN Substancia nigra

SNpc Substancia nigra pars compacta

SoS Sum-of-square

SPM Statistical parametric mapping

TBSS Tract-Based Spatial Statistics

TE Time of echo

TL Temporal lobe

TR Time of repetition

UPDRS Unified Parkinson's Disease Rating Scale

VBA Voxel-based analysis

WM White matter 


\section{List of Figures}

1.1 a) Brain representation of the corpus callosum (lithograph plate from the 20th U.S. edition of Gray's Anatomy of the Human Body). b) Similar view using diffusion imaging tractography. Image from Gigandet et al.[2008) . . . . . . . . . 12

2.1 The main components of the central nervous system. a) Schematic representation of a neuron and the main cellular elements of the white matter, adapted from Johansen-Berg and Behrens $[(2009)$, b) Shematic representation of an axon surrounded by myelin. Electron myograph of c) axonal section and d) longitudinal section of an optic nerve. b), c) and d) are reproduced from Beaulieu (2002) with permissions (Copyright (c) 2002 John Wiley \& Sons, Ltd.) . . . . . . . . . 16

2.2 The conventional PGSE sequence. The first row represents the radiofrequency (Rf) pulses, the second the applied gradients (here for one single direction), the third one the signal and the last one represents the evolution of the phase for two molecules: a non-mobile one (solid line) and a mobile one (dashed line). TE is the echo time, $\mathrm{G}$ the gradient strength, $\delta$ the gradient duration and $\Delta$ the diffusion time. . . . . . . . 17

2.3 a) The echo planar imaging (EPI) readout and b) its corresponding k-space trajectory. Each echo is centered in k-space (adapted from Le Bihan et al. $(2006))]$. . . . . . . . . . . . . 18

2.4 The effect of eddy currents with EPI. a) Effect of eddy cur\begin{tabular}{|l|l|}
\hline rents on the gradient shape. The theoretical gradient is \\
\hline \hline shown in black and the distorted gradient in red. b) k-space \\
\hline \hline trajectory with the presence of eddy currents: echoes are not \\
\hline centered anymore (adapted from Le Bihan et al. (2006) with \\
\hline permissions (Copyright (c) 2006 John Wiley \& Sons, Ltd.)) . & 19 \\
\hline 2.5 & The double refocused spin echo sequence. Use of negative \\
\hline gradients reduces the effects of eddy currents . . . . . . . 20
\end{tabular} 
2.6 Example of a volume corrupted by motion . . . . . . . . . . . 22

2.7 Typical diffusion tensor shapes and FA values found in the three kind of brain tissue . . . . . . . . . . . . . . . 25

2.8 Examples of distributions with different excess kurtosis. The red one $(\mathrm{K}=0)$ corresponds to a Gaussian distribution. . . . . 26

3.1 Schematic representation of the iterative procedure for realignment of diffusion weighted images . . . . . . . . . . . 38

3.2 Estimated movements obtained the different approaches com-

\begin{tabular}{|l|}
\hline pared to the reference, in the case where non-DW images are \\
\hline \hline interleaved with the DW images. The x-axis represents the \\
\hline
\end{tabular}
volume number and can be seen as a time representation. . . 43

3.3 Tensor distance maps for the non-DW interleaved case. . . . 44

3.4 Estimated movements obtained the different approaches com-

pared to the reference, in the case where all non-DW images were acquired before the DW images. The x-axis represents the volume number and can be seen as a time representation. 46

3.5 Tensor distance maps for the case where all non-DW images are acquired before the DW images. ROI1 and ROI2 are indicated in green. . . . . . . . . . . . . . . . . . 47

$3.6 \quad$ Stopping criterion evolution over 20 iterations. . . . . . . . . 49

3.7 Estimated parameters for a real data set. The x-axis represents the volume number and can be seen as a time represen-

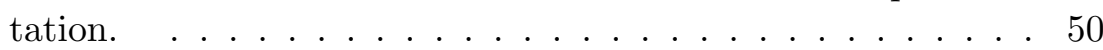

3.8 Tensor distance maps between the three methods . . . . . . . 50

$4.1 \quad$ Probability density function of the signal for different SNR and different acquisitions, (a) Rician distribution can get approximated by a Gaussian distribution with mean $\sqrt{ } A^{2}+\sigma^{2}$ and standard deviation $\sigma$ for higher SNR, (b) Noncentral$\chi$ distributions for sum-of-square reconstruction with multichannels coil $(L=8)$. . . . . . . . . . . . . . . . . 56

$4.2 \quad$ A. Histogram of a noise image with central $\chi$ distribution fit (solid red line). Fitting the histogram with the central $\chi$ distribution gives $\sigma=12,02$ and using the formula $\sigma=12.08$. B. Comparison of the noise estimation on the background of diffusion images compared to noise-only image using the background formula. Estimation on noise-only image is considered as the correct approximation. . . . . . . . . . . . 58

4.3 The different head positions for Protocol 1a. Images are displayed in radiological reference (left is right, right is left) . . . 62 
4.4 Localization of the four different ROIs on a non-DW image

\begin{tabular}{|l|}
\hline in the left in red and in the right in blue. A. Temporal lobe. \\
\hline \hline B. Internal capsule. C. Anterior corona radiata. D. Globus \\
\hline \hline
\end{tabular}
pallidus. . . . . . . . . . . . . . 65

4.5 Normalized signal attenuation curves before and after noise \begin{tabular}{|cc}
\hline corrections together with their DKI fits for the different cor- \\
\hline
\end{tabular} rection schemes. The data correspond to a single diffusion direction in WM areas, for two different voxels with apparent SNR values equal to 26.3 (a) and 16.7 (b) respectively. Corresponding values of $K_{a p p}$ and $D_{a p p}$ are summarized in Table 4.2] ................... . . 67

4.6 Visual inspection of the effect of noise corrections on a DW image $\left(b=1000 \mathrm{~s} / \mathrm{mm}^{2}\right.$. (a) Raw image (NC), (b) M1, (c)M2, (d) BM4D+NC, (e) BM4D+M1, (f) BM4D+M2 . . . . . . . 68

4.7 (a) $M K$ map for one single subject (position P1) and one selected slice corrected with method M1. (b) $M K$, (c) $M D$ and (d) $F A$ histograms of the same slice for each noise correction scheme. .................. . . 69

4.8 (a) $M K$ map of the selected slice for high SNR acquisition (P6) corrected with M1 and (b) the $M K$ histograms of the same slice for three correction schemes (dashed lines). The corresponding histograms for P1 (low SNR acquisition) are also shown (solid curves). The histograms are practically overlapping after the noise correction (blue and green curves). The non-corrected histograms are shifted with respect to each other. However, the difference between corrected and noncorrected values is smaller for higher SNR. . . . . . . . . . . 70

4.9 SNR of non-DWI images and corresponding MK maps for low SNR acquisitions (P1 to P5) and high SNR acquisition (P6). (a) Maps for the 6 positions and 3 different corrections. The region delineated by a white rectangle is zoomed in (b) for 4 selected positions (P1, P4, P5 and P6) and the same correction schemes (shown in color for better visualization). . 77

4.9 SNR of non-DWI images and corresponding MK maps for low SNR acquisitions (P1 to P5) and high SNR acquisition (P6). (a) Maps for the 6 positions and 3 different corrections. The region delineated by a white rectangle is zoomed in (b) for 4 selected positions (P1, P4, P5 and P6) and the same correction schemes (shown in color for better visualization). . 78 
4.10 Averaged MK values for the different correction schemes in two different ROIs (a) Right Globus Pallidus and (b) Left Temporal Lobe. Results are shown for the same subject with 6 different levels of SNR corresponding to acquisitions P1 to P6. . . . . . . . . . . . . . . . 79

4.11 Z-score maps for each correction scheme (rows) and low SNR positions (P1 to P5). The reference is the average MK map over the 5 positions for each correction schemes. Arrows indicate regions of positive bias of MK due to lower SNR. . . . 80

4.12 Averaged MK maps over 25 subjects for one selected slice (first and third rows) and the corresponding standard deviation maps (second and fourth rows) for (a) NC, (b) M1, (c) M2,(d) BM4D+NC, (e) BM4D+M1, (f) BM4D+M2. (g) Mean SNR maps of the averaged non-DW image. . . . . . . . 81

4.13 Histograms of the MK maps averaged over all subjects for all the different correction schemes for one selected slice (the same as in Figure 7): (a) M1, (b) M2, (c) NC, (d) BM4D+M1, (e) BM4D+M2, (f) BM4D+NC. Red and blue lines are the histograms for the WM and GM, respectively. . . . . . . . . . 82

$5.1 \quad$ Significant clusters for all the parameters are displayed on the mean structural MTsat image. FA (green) is lower in patients in a region at the boundaries of the right amygdala (Am), the uncinate fasciculus (UF) and the acoustic radiation (AR) and in the right Posterior thalamic radiation (PTL); MK (red), RK (blue) and AK (yellow) are higher in patients than in controls in the same region where FA is lower (Am, UF, AR), in a region encompassing the right Inferior fronto-occipital fasciculus (IFOF), the UF and a small portion of the putamen (Put), in the right primary auditory cortex (PAC), the left superior parietal lobule (SPL), the right parietal lobe (PL), the right precuneus (Prec), the left primary somatosensory cortex (PSC) and the left primary motor cortex (PMC). . . . 90

5.2 Significant clusters after small volume correction. a) FA is increased in controls in the brainstem and MK, RK and AK are increased in patients in the putamen. . . . . . . . . . 92 


\section{List of Tables}

3.1 Root mean square errors calculated over all volumes for each

\begin{tabular}{|c|}
\hline approach and each parameter with the non-DW interleaved \\
\hline \hline data set
\end{tabular}

3.2 Mean tensor distances after and before motion correction in four different volumes of interest - Case of interleaved non-

ا. DW images . . . . . . . . . . . . . . . 44

3.3 Errors on FA after and before motion correction in four different volumes of interest - Case of interleaved non-DW images 45

3.4 Errors on the main fiber direction after and before motion \begin{tabular}{|c|}
\hline correction in four different volumes of interest - Case of in- \\
\hline terleaved non-DW images
\end{tabular}

3.5 Root mean square errors calculated over all volumes for each approach and each parameter with the non-interleaved data

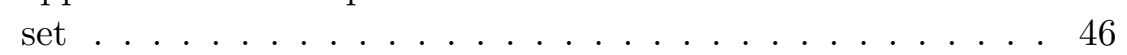

3.6 Mean tensor distances after and before motion correction in four different volumes of interest - Case of non interleaved non-DW images . . . . . . . . . . . . . . . . . 48

3.7 Error on FA after and before motion correction in four dif\begin{tabular}{|c|}
\hline ferent volume of interest - Case of non interleaved non-DW \\
\hline
\end{tabular} images ................... 48

3.8 Error on the main fiber direction after and before motion correction in four different volumes of interest - Case of non interleaved non-DW images . . . . . . . . . . . . 48

4.1 Summary of the parameters of each protocols . . . . . . . . 61

$4.2 \quad K_{a p p}$ and $D_{a p p}\left(10^{-3} \mathrm{~mm}^{2} / \mathrm{s}\right)$ values for two voxels with different SNR, corresponding to Figure 4.5 , with their standard

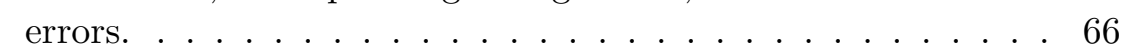


4.3 Mean MK values and standard deviations for each correction scheme and each ROI. Pvalues for Pearson correlation with SNR are indicated by: ${ }^{*} p<0.05$ and ${ }^{* *} p<0.01$. All correlations were negative. . . . . . . . . . . . 71

4.4 Mean MK values and standard deviation over 25 subjects for each correction scheme. . . . . . . . . . . . . . 72

5.1 Test results and information about the groups of patients and healthy controls. Values reflect mean (standard deviation). Two-tail t-tests were performed with an assumption of unequal variance in each group. ICV = Intracranial Volume, BMI = Body Mass Index, LEDD = L-DOPA Equivalent daily dose (Hobson et al., 2002), UPDRS = Unified Parkinson's Disease Rating Scale, MMSE = Mini Mental State Examination, HADS = Hospital Anxiety and Depression Scale, PDQ = Parkinson's Disease Questionnaire, RAVLT=Rey Auditory Verbal Learning Test, SDMT = Symbol Digit Modalities Test, JOLO=Judgment of line orientation test . . . . . . . . 89

5.2 Table of significant clusters where FA is larger in controls . . 89

5.3 Table of significant clusters where MK is larger in patients. . 91

5.4 Table of significant clusters where AK is larger in controls . . 91

5.5 Table of significant clusters where RK is larger in controls . . 91

5.6 Table of significant clusters using region-of-interest masking. P stands for patients and C for controls . . . . . . . . . . . . 92 


\section{Bibliography}

S. Aja-Fernandez and A. Tristan-Vega. Influence of noise correlation in multiple-coil statistical models with sum of squares reconstruction. Magn Reson Med, 67(2):580-5, 2012.

S. Aja-Fernandez, A. Tristan-Vega, and C. Alberola-Lopez. Noise estimation in single- and multiple-coil magnetic resonance data based on statistical models. Magn Reson Imaging, 27(10):1397-409, 2009.

M. Aksoy, C. Forman, M. Straka, S. Skare, S. Holdsworth, J. Hornegger, and R. Bammer. Real-time optical motion correction for diffusion tensor imaging. Magn Reson Med, 66(2):366-378, 2011.

M.E. Alexander, R. Baumgartner, A.R. Summers, C. Windischberger, M. Klarhoefer, E. Moser, and R.L. Somorjai. A wavelet-based method for improving signal-to-noise ratio and contrast in MR images. Magn Reson Imaging, 18(2):169 - 180, 2000.

A. Alhamud, M. Dylan Tisdall, Aaron T. Hess, Khader M. Hasan, Ernesta M. Meintjes, and Andre J.W. van der Kouwe. Volumetric navigators for real-time motion correction in diffusion tensor imaging. Magn Reson Med, 68(4):1097-1108, 2012.

J.L.R. Andersson and S. Skare. A model-based method for retrospective correction of geometric distortions in diffusion-weighted EPI. Neuroimage, 16(1):177 - 199, 2002.

J. Ashburner. A fast diffeomorphic image registration algorithm. Neuroimage, 38(1):95-113, 2007.

J. Ashburner and K.J. Friston. Unified segmentation. Neuroimage, 26(3): $839-851,2005$.

Y. Assaf and P.J. Basser. Composite hindered and restricted model of diffusion (CHARMED) MR imaging of the human brain. Neuroimage, 27 (1):48 - 58, 2005. 
Y. Bai. Correcting for motion between aacquisition in diffusion MR imaging. $\mathrm{PhD}$ thesis, University College London, 2009.

Yu Bai and D.C. Alexander. Model-based registration to correct for motion between acquisitions in diffusion MR imaging. In Biomedical Imaging: From Nano to Macro, 2008. ISBI 2008. 5th IEEE International Symposium on, pages 947-950, 2008.

A. E. Baird and S. Warach. Magnetic resonance imaging of acute stroke. $J$ Cereb Blood Flow Metab, 18:583-609, 1998.

P. J. Basser, J. Mattiello, and D. LeBihan. Estimation of the effective self-diffusion tensor from the NMR spin echo. J Magn Reson B, 103(3): 247-54, 1994.

P.J. Basser. Inferring microstructural features and the physiological state of tissues from diffusion-weighted images. NMR Biomed, 8:333-44, 1995.

C. Beaulieu. The basis of anisotropic water diffusion in the nervous system, a technical review. NMR in Biomedicine, 15(7-8):435-455, 2002.

S. Ben-Amitay, D. K. Jones, and Y. Assaf. Motion correction and registration of high b-value diffusion weighted images. Magn Reson Med, 67(6): 1694-1702, 2012.

T. Benner, Andre J. W. van der Kouwe, and A. G. Sorensen. Diffusion imaging with prospective motion correction and reacquisition. Magn Reson Med, 66(1):154-167, 2011.

A. L. Benton, N. R. Varney, and K. S. Hamsher. Visuospatial judgment: A clinical test. Arch. Neurol., 35:364-367, 1978.

M.A. Bernstein, Thomasson David M., and Perman William H. Improved detectability in low signal-to-noise ratio magnetic resonance images by means of a phase-corrected real reconstruction. $\mathrm{Med} P h, 16: 813-17,1989$.

I. Blockx, M. Verhoye, J. Van Audekerke, I. Bergwerf, J. X. Kane, Y. Palacios R. Delgado, J. Veraart, B. Jeurissen, K. Raber, S. von Horsten, P. Ponsaerts, J. Sijbers, T. B. Leergaard, and A. Van der Linden. Identification and characterization of Huntington related pathology: an in vivo DKI imaging study. Neuroimage, 63(2):653-62, 2012.

M.D. Boska, K.M. Hasan, D. Kibuule, R. Banerjee, E. McIntyre, J.A. Nelson, T. Hahn, H.E. Gendelman, and R.L. Mosley. Quantitative diffusion tensor imaging detects dopaminergic neuronal degeneration in a murine model of Parkinson's disease. Neurobiol Dis., 26(3):590 - 596, 2007. 
Édouard Brissaud. Leçons sur les maladies nerveuses, volume 2. Masson, 1899.

L. G. Brown. A survey of image registration techniques. ACM Comput. Surv., 24(4):325-376, December 1992.

R. Brown. A brief account of microscopical observations made in the months of june, july and august, 1827, on the particles contained in the pollen of plants; and on the general existence of active molecules in organic and inorganic bodies. Philosophical Magazine, 4:161-173, 1828.

M. E. Brummer, R. M. Mersereau, R. L. Eisner, and R. J. Lewine. Automatic detection of brain contours in MRI data sets. IIEEE Trans Med Imaging., 12(2):153-66, 1993.

RE. Burke and O'Malley K. Axon degeneration in Parkinson's disease. Experimental Neurology, 246(0):72 - 83, 2013.

F. Calamante, J.D. Tournier, G.D. Jackson, and A. Connelly. Track-density imaging (tdi): Super-resolution white matter imaging using whole-brain track-density mapping. Neuroimage, 53(4):1233 - 1243, 2010.

B.C. Carlson. Computing elliptic integrals by duplication. Numerische Mathematik, 33(1):1-16, 1979.

L.C. Chang, D.K. Jones, and C. Pierpaoli. Restore: Robust estimation of tensors by outlier rejection. Magn Reson Med, 53(5):1088-1095, 2005.

T L Chenevert, J A Brunberg, and J G Pipe. Anisotropic diffusion in human white matter: demonstration with MR techniques in vivo. Radiology, 177 (2):401-405, 1990.

S. Chung, B. Courcot, M. Sdika, K. Moffat, C. Rae, and R. G. Henry. Bootstrap quantification of cardiac pulsation artifact in DTI. Neuroimage, 49(1):631-40, 2010.

C.J. Cochrane and K.P. Ebmeier. Diffusion tensor imaging in parkinsonian syndromes: a systematic review and meta-analysis. Neurology, 80:857-64, 2013.

A. Collard. Geometric statistical processing of brain diffusion tensor images. PhD thesis, University of Liège, 2013.

A. Collard, S. Bonnabel, C. Phillips, and R. Sepulchre. Anisotropy preserving DTI processing. Int J Comput Vis, 107:58-74, 2014. 
C. D. Constantinides, E. Atalar, and E. R. McVeigh. Signal-to-noise measurements in magnitude images from NMR phased arrays. Magn Reson Med, 38(5):852-7, 1997.

T. E. Conturo, N.F. Lori, T.S. Cull, E. Akbudak, A. Z. Snyder, J. S. Shimony, R. C. McKinstry, H. Burton, and M. E. Raichle. Tracking neuronal fiber pathways in the living human brain. Proc Natl Acad Sci US A, 96 (18):10422-10427, 1999.

P. Coupe, P. Yger, S. Prima, P. Hellier, C. Kervrann, and C. Barillot. An optimized blockwise nonlocal means denoising filter for 3-D magnetic resonance images. IEEE Trans Med Imaging., 27(4):425-441, 2008.

W R Crum, T Hartkens, and D L G Hill. Non-rigid image registration: theory and practice. Br. J. Radiol., 77(suppl 2):S140-S153, 2004.

S. De Santis, Y. Assaf, and D. K. Jones. Using the biophysical CHARMED model to elucidate the underpinnings of contrast in diffusional kurtosis analysis of diffusion-weighted MRI. Magma, 2011.

A. Deshmane, V. Gulani, M. A. Griswold, and N. Seiberlich. Parallel MR imaging. J Magn Reson Imaging, 36(1):55-72, 2012.

O. Dietrich, J.G. Raya, S.B. Reeder, M.F. Reiser, and S.O. Schoenberg. Measurement of signal-to-noise ratios in MR images: Influence of multichannel coils, parallel imaging, and reconstruction filters. J Magn Reson Imaging, 26(2):375-385, 2007.

B. Draganski, J. Ashburner, C. Hutton, F. Kherif, R.S.J. Frackowiak, G. Helms, and N. Weiskopf. Regional specificity of MRI contrast parameter changes in normal ageing revealed by voxel-based quantification (VBQ). Neuroimage, 55(4):1423 - 1434, 2011.

A. Einstein. Uber die von der molecularkinetischen theorie der warme geforderte bewegung von in ruhenden fluessigkeiten suspendierten teilchen. Ann. Phys. (Leipzig), 17:549-569, 1905.

S. Fahn and RL. UPDRS program members. Elton. Recent developments in Parkinsons disease, vol 2., chapter Unified Parkinsons Disease Rating Scale., page 153. Florham Park, NJ: Macmillan Healthcare Information, 1987.

M. F. Falangola, J. H. Jensen, J. S. Babb, C. Hu, F. X. Castellanos, A. Di Martino, S. H. Ferris, and J. A. Helpern. Age-related non-gaussian diffusion patterns in the prefrontal brain. J Magn Reson Imaging, 28(6): 1345-50, 2008. 
M. F. Falangola, J. H. Jensen, A. Tabesh, C. Hu, R. L. Deardorff, J. S. Babb, S. Ferris, and J. A. Helpern. Non-gaussian diffusion MRI assessment of brain microstructure in mild cognitive impairment and Alzheimer's disease. Magn Reson Imaging, 2013.

E. Fieremans, J. H. Jensen, and J. A. Helpern. White matter characterization with diffusional kurtosis imaging. Neuroimage, 58:177-88, 2011.

C. Foix and J. Nicolesco. Anatomie cerebrale: les noyaux gris Centraux et la region Mesencephalo-soux-optique. Masson, Paris, 1925.

M.F. Folstein, S.E. Folstein, and P.R. McHugh. "Mini-mental state": A practical method for grading the cognitive state of patients for the clinician. J Psychiatr Res, 12(3):189 - 198, 1975.

K. Friston, J. Ashburner, S. Kiebel, T. Nichols, and W. Penny. Statistical Parametric Mapping: the analysis of functional brain images. Elsevier Academic Press, London, 2007.

S. Furlan. Spatial realignment of diffusion weighted magnetic resonance images. Master's thesis, University of Liège, 2011.

Y. Gao, Y. Zhang, C. S. Wong, P. M. Wu, Z. Zhang, J. Gao, D. Qiu, and B. Huang. Diffusion abnormalities in temporal lobes of children with temporal lobe epilepsy: a preliminary diffusional kurtosis imaging study and comparison with diffusion tensor imaging. NMR Biomed, 25(12): 1369-77, 2012.

G. Gerig, O. Kubler, R. Kikinis, and F.A. Jolesz. Nonlinear anisotropic filtering of MRI data. IEEE Trans Med Imaging., 11(2):221-232, 1992.

X. Gigandet, P. Hagmann, M. Kurant, L. Cammoun, R Meuli, and J.P. Thiran. Estimating the confidence level of white matter connections obtained with MRI tractography. PloS ONE, 3:e4006, 2008.

H.M. Golshan, R.P.R. Hasanzadeh, and S.C. Yousefzadeh. An MRI denoising method using image data redundancy and local SNR estimation. $J$ Magn Reson Imaging, 31:1206-17, 2013.

F. Grinberg, E. Farrher, J. Kaffanke, A. M. Oros-Peusquens, and N. J. Shah. Non-gaussian diffusion in human brain tissue at high b-factors as examined by a combined diffusion kurtosis and biexponential diffusion tensor analysis. Neuroimage, 57(3):1087-102, 2011. 
F. Grinberg, L. Ciobanu, E. Farrher, and N. J. Shah. Diffusion kurtosis imaging and log-normal distribution function imaging enhance the visualisation of lesions in animal stroke models. NMR Biomed, 25(11): 1295-304, 2012.

E. J. Grossman, Y. Ge, J. H. Jensen, J. S. Babb, L. Miles, J. Reaume, J. M. Silver, R. I. Grossman, and M. Inglese. Thalamus and cognitive impairment in mild traumatic brain injury: A diffusional kurtosis imaging study. J Neurotrauma, 2011.

H. Gudbjartsson and S. Patz. The Rician distribution of noisy MRI data. Magn Reson Med, 34(6):910-4, 1995.

E. L. Hahn. Spin echoes. Phys. Rev., 80:580-594, 1950.

K.M. Hasan, D.L. Parker, and A.L. Alexander. Comparison of gradient encoding schemes for diffusion-tensor MRI. J Magn Reson Imaging, 13 (5):769-780, 2001.

J. C. Haselgrove and J. R. Moore. Correction for distortion of echo-planar images used to calculate the apparent diffusion coefficient. Magn Reson Med, 36(6):960-964, 1996.

J. A. Helpern, V. Adisetiyo, M. F. Falangola, C. Hu, A. Di Martino, K. Williams, F. X. Castellanos, and J. H. Jensen. Preliminary evidence of altered gray and white matter microstructural development in the frontal lobe of adolescents with attention-deficit hyperactivity disorder: a diffusional kurtosis imaging study. J Magn Reson Imaging, 33(1):17-23, 2011.

R. M. Henkelman. Measurement of signal intensities in the presence of noise in MR images. Med Phys, 12(2):232-3, 1985.

D.E. Hobson, A.E. Lang, W. Martin, A. Razmy, J. Rivest, and J. Fleming. Excessive daytime sleepiness and sudden-onset sleep in parkinson disease: A survey by the canadian movement disorders group. JAMA, 287(4):455463, 2002.

M. Hoehn and M. Yahr. Parkinson: onset, progression and mortality. Neurology, 17:427-42, 1967.

D.I. Hoult and P.C. Lauterbur. The sensitivity of the zeugmatographic experiment involving human samples. J Magn Reson, 34:425-433, 1979.

E. S. Hui, M. M. Cheung, L. Qi, and E. X. Wu. Towards better MR characterization of neural tissues using directional diffusion kurtosis analysis. Neuroimage, 42(1):122-34, 2008. 
C. Hutton, E. Balteau, A. Lutti, O. Josephs, and N. Weiskopf. Modelling temporal stability of EPI time series using magnitude images acquired with multi-channel receiver coils. PLoS One, 7(12):e52075, 2012.

M. Jahanshahi, T. Saleem, A. K. Ho, G. Dirnberger, and R Fuller. Random number generation as an index of controlled processing. Neuropsychology, 20:391-399, 2006.

C. Jenkinson, R. Fitzpatrick, V. Peto, R. Greenhall, and N. Hyman. The Parkinson's disease questionnaire (PDQ-39): development and validation of a Parkinson's disease summary index score. Age and Ageing, 26(5): 353-357, 1997.

M. Jenkinson, C. F. Beckmann, T. E. Behrens, M. W. Woolrich, and S. M. Smith. Fsl. Neuroimage, 62(2):782-90, 2012.

J. H. Jensen and J. A. Helpern. MRI quantification of non-gaussian water diffusion by kurtosis analysis. NMR Biomed, 23(7):698-710, 2010.

J. H. Jensen, J. A. Helpern, A. Ramani, H. Lu, and K. Kaczynski. Diffusional kurtosis imaging: the quantification of non-gaussian water diffusion by means of magnetic resonance imaging. Magn Reson Med, 53(6):143240, 2005.

P. Jezzard and R. S. Balaban. Correction for geometric distortion in echo planar images from b0 field variations. Magn Reson Med, 34(1):65-73, 1995.

P. Jezzard, A. S. Barnett, and C. Pierpaoli. Characterization of and correction for eddy current artifacts in echo planar diffusion imaging. Magn Reson Med, 39(5):801-812, 1998.

H. Johansen-Berg and T.E.J. Behrens. Diffusion MRI. Elsevier, 2009.

D. K. Jones. The effect of gradient sampling schemes on measures derived from diffusion tensor MRI: A monte carlo study. Magn Reson Med, 51 (4):807-815, 2004.

D. K. Jones and P. J. Basser. "Squashing peanuts and smashing pumpkins": how noise distorts diffusion-weighted MR data. Magn Reson Med, 52(5): 979-93, 2004.

D.K. Jones and M. Cercignani. Twenty-five pitfalls in the analysis of diffusion MRI data. NMR in Biomedicine, 23(7):803-820, 2010. 
D.K. Jones, M.A. Horsfield, and A. Simmons. Optimal strategies for measuring diffusion in anisotropic systems by magnetic resonance imaging. Magn Reson Med, 42(3):515-525, 1999.

K. Kamagata, H. Tomiyama, Y. Motoi, M. Kano, O. Abe, K. Ito, K. Shimoji, M. Suzuki, M. Hori, A. Nakanishi, R. Kuwatsuru, K. Sasai, S. Aoki, and N. Hattori. Diffusional kurtosis imaging of cingulate fibers in parkinson disease: Comparison with conventional diffusion tensor imaging. Magn Reson Imaging, 31:1501-6, 2013.

SL. Keeling. Total variation based convex filters for medical imaging. Appl. Math. Comput, 139:101-119, 2003.

C. G. Koay. Diffusion MRI: theory, methods and applications, chapter Least squares approaches to diffusion tensor estimation, pages 272-284. 2011.

C. G. Koay and P. J. Basser. Analytically exact correction scheme for signal extraction from noisy magnitude MR signals. J Magn Reson, 179 (2):317-22, 2006.

C. G. Koay, E. Ozarslan, and P. J. Basser. A signal transformational framework for breaking the noise floor and its applications in MRI. J Magn Reson, 197(2):108-19, 2009.

T. Kober, R. Gruetter, and G Krueger. Prospective and retrospective motion correction in diffusion magnetic resonance imaging of the human brain. Neuroimage, 59(1):389 - 398, 2012.

A. Kristoffersen. Estimating non-gaussian diffusion model parameters in the presence of physiological noise and rician signal bias. J Magn Reson Imaging, 35(1):181-9, 2012.

F. Lam, S. D. Babacan, J.P. Haldar, M.W. Weiner, N. Schuff, and Z.P. Liang. Denoising diffusion-weighted magnitude MR images using rank and edge constraints. Magn Reson Med, pages n/a-n/a, 2013.

J. Latt, M. Nilsson, R. Wirestam, F. Stahlberg, N. Karlsson, M. Johansson, P. C. Sundgren, and D. van Westen. Regional values of diffusional kurtosis estimates in the healthy brain. J Magn Reson Imaging, 37(3):610-8, 2013.

D. Le Bihan, E. Breton, D. Lallemand, P. Grenier, E. Cabanis, and M. Laval-Jeantet. MR imaging of intravoxel incoherent motions: application to diffusion and perfusion in neurologic disorders. Radiology, 161(2):401-407, 1986. 
D. Le Bihan, C. Poupon, A. Amadon, and F. Lethimonnier. Artifacts and pitfalls in diffusion MRI. J Magn Reson Imaging, 24(3):478-488, 2006.

A. Leemans and D. K. Jones. The b-matrix must be rotated when correcting for subject motion in DTI data. Magn Reson Med, 61(6):1336-49, 2009.

A. Leemans, B. Jeurissen, J. Sijbers, and D.K. Jones. ExploreDTI: a graphical toolbox for processing, analyzing, and visualizing diffusion MR data. 17th Annual Meeting of Intl Soc Mag Reson Med, Hawaii, USA:3537, 2009.

G. Llebaria, J. Pagonabarraga, J. Kulisevsky, C. Garcia-Sanchez, B. Pascual-Sedano, A. Gironell, and M. Martinez-Corral. Cut-off score of the mattis dementia rating scale for screening dementia in Parkinson's disease. Mov Disord., 23(11):1546-1550, 2008.

H. Lu, J. H. Jensen, A. Ramani, and J. A. Helpern. Three-dimensional characterization of non-gaussian water diffusion in humans using diffusion kurtosis imaging. NMR in biomedicine, 19(2):236-47, 2006.

A. Macovski. Noise in MRI. Magn Reson Med, 36(3):494-7, 1996.

M. Maggioni, V. Katkovnik, K. Egiazarian, and A. Foi. A nonlocal transform-domain filter for volumetric data denoising and reconstruction. IEEE Trans Image Process, 2012.

M. Maggioni, V. Katkovnik, K. Egiazarian, and A. Foi. Nonlocal transformdomain filter for volumetric data denoising and reconstruction. IEEE Trans Image Process, 22(1):119-133, 2013.

J. V. Manjon, P. Coupe, L. Marti-Bonmati, D. L. Collins, and M. Robles. Adaptive non-local means denoising of MR images with spatially varying noise levels. J Magn Reson Imaging, 31(1):192-203, 2010.

J. Mattiello, PJ. Basser, and D. Le Bihan. The b-matrix in diffusion tensor echo-planar imaging. Magn Reson Med, 37:292-300, 1997.

II Maximov, F. Grinberg, and N. J. Shah. Robust tensor estimation in diffusion tensor imaging. J Magn Reson, 213(1):136-44, 2011.

II Maximov, E. Farrher, F. Grinberg, and N. J. Shah. Spatially variable rician noise in magnetic resonance imaging. Med Image Anal, 16(2):536$48,2012$.

G. McGibney and M.R. Smith. An unbiased signal-to-noise ratio measure for magnetic resonance images. Med. Phys., 20:1077, 1993. 
T.R. Mhyre, J.T. Boyd, R.W. Hamill, and K.A. Maguire-Zeiss. Parkinsons disease. In J. Robin Harris, editor, Protein Aggregation and Fibrillogenesis in Cerebral and Systemic Amyloid Disease, volume 65 of Subcellular Biochemistry, pages 389-455. Springer Netherlands, 2012. ISBN 978-94007-5415-7.

A. J. Miller and P. M. Joseph. The use of power images to perform quantitative analysis on low SNR MR images. Magn Reson Imaging, 11(7): 1051-6, 1993.

S. Mohammadi, H. E. Moller, H. Kugel, D. K. Mueller, and M. Deppe. Correcting eddy current and motion effects by affine whole-brain registrations: Evaluation of three-dimensional distortions and comparison with slicewise correction. Magn Reson Med, 64(4):1047-1056, 2010.

S. Mori, B.J. Crain, V.P. Chacko, and P.C.M. Van Zijl. Three-dimensional tracking of axonal projections in the brain by magnetic resonance imaging. Ann Neurol, 45:265-269, 1999.

M E Moseley, Y Cohen, J Kucharczyk, J Mintorovitch, H S Asgari, M F Wendland, J Tsuruda, and D Norman. Diffusion-weighted MR imaging of anisotropic water diffusion in cat central nervous system. Radiology, 176(2):439-445, 1990.

R. V. Mulkern, S. J. Haker, and S. E. Maier. On high b diffusion imaging in the human brain: ruminations and experimental insights. Magn Reson Imaging, 27(8):1151-62, 2009.

B.W. Murphy, P.L. Carson, J.H. Ellis, Y.T. Zhang, R.J. Hyde, and T LT.L. Chenevert. Signal-to-noise measures for magnetic resonance imagers. Magn Reson Imaging, 11(3):425 - 428, 1993.

H. Nam and H.J. Park. Distortion correction of high b-valued and high angular resolution diffusion images using iterative simulated images. Neuroimage, 57(3):968 - 978, 2011.

NEMA. Determination of signal-to-noise ratio in diagnostic magnetic resonance imaging. NEMA Standard Publication no. MS 1-2001, National Electrical Manufacturers Association, Rosslyn, Va, USA, 2001.

H. Ni, V. Kavcic, T. Zhu, S. Ekholm, and J. Zhong. Effects of number of diffusion gradient directions on derived diffusion tensor imaging indices in human brain. AJNR Am J Neuroradiol, 27(8):1776-1781, 2006. 
T. Niendorf, R. M. Dijkhuizen, D. G. Norris, M. van Lookeren Campagne, and K. Nicolay. Biexponential diffusion attenuation in various states of brain tissue: implications for diffusion-weighted imaging. Magn Reson Med, 36(6):847-57, 1996.

R. D. Nowak. Wavelet-based rician noise removal for magnetic resonance imaging. IEEE Trans Image Process, 8(10):1408-19, 1999.

R. G. Nunes, P. Jezzard, and S. Clare. Investigations on the efficiency of cardiac-gated methods for the acquisition of diffusion-weighted images. $J$ Magn Reson, 177(1):102-10, 2005.

N.G. Papadakis, C.D. Murrills, L.D. Hall, C.L. Huang, and T.A. Carpenter. Minimal gradient encoding for robust estimation of diffusion anisotropy. Magn Reson Imaging, 18(6):671 - 679, 2000.

D.L. Parker and G.T. Gullberg. Signal-to-noise efficiency in magnetic resonance imaging. Med. Phys., 17:250-7, 1990.

P. Raab, E. Hattingen, K. Franz, F. E. Zanella, and H. Lanfermann. Cerebral gliomas: diffusional kurtosis imaging analysis of microstructural differences. Radiology, 254(3):876-81, 2010.

J.G. Raya, V. Manjon, J.J. Carbonell-Caballero, J.and Lull, G. GarciaMarti, L. Marti-Bonmati, and M. Robles. MRI denoising using non-local means. Med Image Anal, 12(4):514 - 523, 2008.

T.G. Reese, O. Heid, R.M. Weisskoff, and V.J. Wedeen. Reduction of eddycurrent-induced distortion in diffusion MRI using a twice-refocused spin echo. Magn Reson Med, 49(1):177-182, 2003.

A. Rey. L'examen clinique en psychologie (The Clinical Examination in Psychology). Presses Universitaires de France, Paris, France, 1964.

G.K. Rohde, A.S. Barnett, P.J. Basser, S. Marenco, and C. Pierpaoli. Comprehensive approach for correction of motion and distortion in diffusionweighted MRI. Magn Reson Med, 51(1):103-114, 2004.

J. Sijbers, A. J. den Dekker, J. Van Audekerke, M. Verhoye, and D. Van Dyck. Estimation of the noise in magnitude MR images. Magn Reson Imaging, 16(1):87-90, 1998.

S. Skare, M. Hedehus, M.E. Moseley, and T.Q. Li. Condition number as a measure of noise performance of diffusion tensor data acquisition schemes with MRI. J Magn Reson, 147(2):340 - 352, 2000. 
S.M. Smith, M. Jenkinson, M.W. Woolrich, C.F. Beckmann, T.E.J. Behrens, H. Johansen-Berg, P.R. Bannister, M. De Luca, I. Drobnjak, D.E. Flitney, R.K. Niazy, J. Saunders, J. Vickers, Y. Zhang, N. De Stefano, J.M. Brady, and P.M. Matthews. Advances in functional and structural MR image analysis and implementation as FSL. Neuroimage, 23, Supplement 1(0):S208 - S219, 2004.

S.M. Smith, M. Jenkinson, H. Johansen-Berg, D. Rueckert, T.E. Nichols, C.E. Mackay, K.E. Watkins, O. Ciccarelli, M.Z. Cader, P.M. Matthews, and T.E.J. Behrens. Tract-based spatial statistics: Voxelwise analysis of multi-subject diffusion data. Neuroimage, 31(4):1487 - 1505, 2006.

E.O. Stejskal and J.E. Tanner. Spin diffusion measurements: Spin echoes in the presence of a time?dependent field gradient. J Chem Phys, 42:288, 1965 .

A. Jon. Stoessl, W.R. Wayne. Martin, Martin J. McKeown, and Vesna Sossi. Advances in imaging in Parkinson's disease. Lancet Neurol., 10:987-1001, 2011.

C. Studholme, D.L.G. Hill, and D.J. Hawkes. An overlap invariant entropy measure of 3d medical image alignment. Pattern Recognition, 32(1):71 86, 1999.

F. Szczepankiewicz, J. Latt, R. Wirestam, A. Leemans, P. Sundgren, D. van Westen, F. Stahlberg, and M. Nilsson. Variability in diffusion kurtosis imaging: Impact on study design, statistical power and interpretation. Neuroimage, 76:145-54, 2013.

P. Thunberg and P. Zetterberg. Noise distribution in SENSE- and GRAPPA-reconstructed images: a computer simulation study. Magn Reson Imaging, 25:1089-94, 2007.

C. Tretiakoff. Contributions a l'étude de l'anatomie pathologique du locus niger de soemmering avec quelques déductions relatives a la pathogénie des troubles de tonus musculaire et de la maladie de Parkinson. $\mathrm{PhD}$ thesis, 1919.

A. Tristan-Vega, V. Garcia-Perez, S. Aja-Fernandez, and C. F. Westin. Efficient and robust nonlocal means denoising of MR data based on salient features matching. Comput Methods Programs Biomed, 105(2):131-44, 2012 .

D.S. Tuch. Q-ball imaging. Magn Reson Med, 53:1358-1372, 2004. 
S. Van Cauter, J. Veraart, J. Sijbers, R. R. Peeters, U. Himmelreich, F. De Keyzer, S. W. Van Gool, F. Van Calenbergh, S. De Vleeschouwer, W. Van Hecke, and S. Sunaert. Gliomas: diffusion kurtosis MR imaging in grading. Radiology, 263(2):492-501, 2012.

J. Veraart, D. H. Poot, W. Van Hecke, I. Blockx, A. Van der Linden, M. Verhoye, and J. Sijbers. More accurate estimation of diffusion tensor parameters using diffusion kurtosis imaging. Magn Reson Med, 65(1):138-45, 2011.

J. Veraart, J. Rajan, R. R. Peeters, A. Leemans, S. Sunaert, and J. Sijbers. Comprehensive framework for accurate diffusion MRI parameter estimation. Magn Reson Med, 70:972-84, 2013.

P. Viola and W.M.I.I.I. Wells. Alignment by maximization of mutual information. In Computer Vision, 1995. Proceedings., Fifth International Conference on, pages 16-23, 1995. doi: 10.1109/ICCV.1995.466930.

S. Volz, U. Nöth, A. Rotarska-Jagiela, and R. Deichmann. A fast b1mapping method for the correction and normalization of magnetization transfer ratio maps at 3T. Neuroimage, 49(4):3015 - 3026, 2010.

J. J. Wang, W. Y. Lin, C. S. Lu, Y. H. Weng, S. H. Ng, C. H. Wang, H. L. Liu, R. H. Hsieh, Y. L. Wan, and Y. Y. Wai. Parkinson's disease: diagnostic utility of diffusion kurtosis imaging. Radiology, 261(1):210-7, 2011.

V.J. Wedeen, R.P. Wang, J.D. Schmahmann, T. Benner, W.Y. Tseng, G. Dai, D.N. Pandya, P. Hagmann, H. D Arceuil, and A.J. De Crespigny. Diffusion spectrum magnetic resonance imaging (DSI) tractography of crossing fibers. Neuroimage, 41:1267-1277, 2008.

N. Weiskopf and G. Helms. Multi-parameter mapping of the human brain at $1 \mathrm{~mm}$ resolution in less than 20 minutes. 16th Annual Meeting of Intl Soc Mag Reson Med, Toronto, CA:2241, 2008.

W.C.K. Wong, A.C.S. Chung, and S.C.H. Yu. Trilateral filtering for biomedical images. In Biomedical Imaging: Nano to Macro, 2004. IEEE International Symposium on, pages 820-823 Vol. 1, 2004 . doi: 10.1109/ISBI.2004.1398664.

J. C. Wood and K. M. Johnson. Wavelet packet denoising of magnetic resonance images: Importance of rician noise at low SNR. Magn Reson Med, 41(3):631-635, 1999. 
V. L. Yarnykh. Actual flip-angle imaging in the pulsed steady state: A method for rapid three-dimensional mapping of the transmitted radiofrequency field. Magn Reson Med, 57(1):192-200, 2007.

E. Ziegler, M. Rouillard, E. André, T. Coolen, J. Stender, E. Balteau, C. Phillips, and G Garraux. Mapping track density changes in nigrostriatal and extranigral pathways in Parkinson's disease. Submitted, 2014.

A.S. Zigmond and R.P. Snaith. The hospital anxiety and depression scale. Acta Psychiatr Scand, 6:361-70, 1983.

B. Zitova and J. Flusser. Image registration methods: a survey. Image Vis Comput, 21(11):977 - 1000, 2003. 


\section{Acknowledgments}

This thesis represents the work I have been conducting over the past four years in the Cyclotron Research Center at the University of Liège, and partly in the Juelich Forschungzentrum. These years have been very rewarding, both personally and professionally. This experience would not have been the same without all the people I have met during that time, and those I already knew.

To start, I would like to thank my supervisors from Liège, Dr. Christophe Phillips, Dr. Evelyne Balteau and Dr. Farida Grinberg for their support, advice and help. Their experience in different fields of neuroimaging has been very complementary and has contributed to the success of this work. Thank you also to Dr. Gaetan Garraux, for his expertise in Parkinson's disease and his helpful insights. I would also like to thank Prof. André Luxen and Prof. Jon. N. Shah for welcoming me in their respective laboratories, and giving me the opportunity to conduct this research in a competitive environment.

My thanks also go to Dr. Ezequiel Farrher, Dr. Ivan Maximov and Erik Ziegler for helpful discussions and brainstorming.

I also want to thank all my colleagues at the CRC. You all participated in the creation of this work, directly or indirectly. In particular, thank you to Maud, Christelle, Mathieu and Vincenzo for acquiring data. Thanks also to all the anonymous participants, without whom none of this work would have been possible.

I also want to thank all team members in Juelich with whom I had very interesting discussions at the coffee corner or during lunch time. A big thanks to Xavier and Daniel for driving me to Juelich.

Thanks also to the M-Bic Graduate school members and to the organizers, for the great workshops and socializing events.

I would also like to thank all my friends, who have always been present, in good times and bad times, for a coffee, an evening or a skype talk! A big thanks also goes out to my family, who has always supported me in all my decisions and without whom I wouldn't be here. Finally, thanks to Ruben for cheering me up more than once, and for brightening my life every day! 


\section{Curriculum vitae}

Elodie André was born on December $16^{\text {th }}$, 1984 in Angers, France. She obtained her high school diploma in June, 2002. She then went to preparatory school in Lycée Clemenceau in Nantes to prepare for the nationwide competition to enter engineering school. In 2004, she moved to Grenoble to follow a program in physics and biomedical engineering at the Grenoble Institute of Technology (INPG), which she graduated from in 2008.

During this time, she spent one year at Imperial College London as an exchange student from September 2006 until May 2007. She also spent three months in Clermont-Ferrand in a medical instrumentation company (CLERAD) to model the particle's path in a $\gamma$-ray detector and optimizing the probes and the imaging system. She did her Master's thesis over six months in Eindhoven in the Netherlands at Philips Research under the supervision of Prof. Dr. Michel Decré. The project title was "Elaboration of compartmented microfluidic systems for in vitro experiments of neural networks".

She then moved to Sweden and spent three months in the Developmental Genetics Unit of the Department of Neuroscience at Uppsala University to study nervous system function with genetic tools in mice. Next she became a research assistant and started a Ph.D. in 2009 in the Department of Clinical Neuroscience at the Karolinksa Institute in Stockholm. She worked on gender-based differences of structural and functional connectivity in the human brain using MRI.

In 2010, she moved to Belgium and started a new $\mathrm{PhD}$ project at the $\mathrm{Cy}-$ clotron Research Center of the University of Liège, as part as a European collaboration with Maastricht University, Juelich Forchungszentrum in Germany, and GlaxoSmithKline in the United Kingdom. 